\title{
The BATSE 5B Gamma-Ray Burst Spectral Catalog
}

\author{
Adam Goldstein ${ }^{1}$, Robert D. Preece ${ }^{1}$, Robert S. Mallozzi*, Michael S. Briggs ${ }^{1}$, \\ Gerald J. Fishman ${ }^{2}$, Chryssa Kouveliotou ${ }^{2}$, William S. Paciesas ${ }^{3}$, J. Michael Burgess ${ }^{1}$
}

Received __; accepted _

\footnotetext{
${ }^{1}$ University of Alabama in Huntsville, 320 Sparkman Drive, Huntsville, AL 35899, USA ${ }^{2}$ Space Science Office, VP62, NASA/Marshall Space Flight Center, Huntsville, AL 35812, USA

${ }^{3}$ Universities Space Research Association, 320 Sparkman Drive, Huntsville, AL 35805, USA

*deceased
} 


\begin{abstract}
We present systematic spectral analyses of GRBs detected with the Burst and Transient Source Experiment (BATSE) onboard the Compton Gamma-Ray Observatory (CGRO) during its entire nine years of operation. This catalog contains two types of spectra extracted from 2145 GRBs, and fitted with five different spectral models resulting in a compendium of over 19000 spectra. The models were selected based on their empirical importance to the spectral shape of many GRBs, and the analysis performed was devised to be as thorough and objective as possible. We describe in detail our procedures and criteria for the analyses, and present the bulk results in the form of parameter distributions. This catalog should be considered an official product from the BATSE Science Team, and the data files containing the complete results are available from the High-Energy Astrophysics Science Archive Research Center (HEASARC, http: //heasarc.gsfc.nasa.gov/W3Browse/cgro/bat5bgrbsp.html).
\end{abstract}

Subject headings: gamma rays: bursts - methods: data analysis 


\section{Introduction}

Gamma-ray bursts (GRBs) are intense flashes of radiation that appear at unpredictable times from random locations on the celestial sphere. They have been studied since their serendipitous discovery by the Vela Satellite Network (Klebesadel, Strong, \& Olson 1973). Despite nearly 40 years of research, the progenitors of these astrophysical phenomena remain largely unknown. Several thousand of these events have been detected by many different instruments. The bulk of the energy emission of GRBs occurs in the X-ray and gamma-ray regime of the electromagnetic spectrum. Their intensities and durations span many orders of magnitude, where the intensity of a burst is usually defined by its peak flux at a specified time resolution and in a specified energy range. The strong bursts are distributed homogeneously in space as the angular distribution is consistent with an isotropic distribution (Meegan et al. 1992; Briggs et al. 1994, 1996). Observations of burst afterglow at other wavelengths may provide substantial evidence of the burst mechanism. Research in this area involves attempting to determine the celestial locations of bright bursts while they are in progress and to distribute these to observers for rapid follow-up observations at other wavelengths (Barthelmy et al. 1994). This afterglow search has yielded several observations of GRB afterglows (e.g.; De Pasquale et al. 2006; Roming et al. 2009).

The count rate time history of each GRBs is unique, rendering classifications based on morphology unsuccessful [see Hrabovsky et al. (1996) for a review]. The shapes of GRB light curves vary widely; they include single-peak and multi-peak events, long duration emission at low intensity, and rapidly rising time profiles with an exponential-like decay phase. Temporal variability on time scales of the order of milliseconds has been observed, although smooth light curves with little variability are also observed (Bhat et al. 1992 ; Lloyd-Ronning \& Ramirez-Ruiz 2002). The study of this variability has lead to theoretical 
development of the internal emission processes of GRBs (Sari \& Piran 1997; Dermer 1998) The durations of bursts span a wide range, with the shortest bursts of the order of milliseconds, and the longest duration bursts lasting for hundreds of seconds. The logarithmic duration distribution of GRBs observed by BATSE appears to exhibit two clusters centered at $\sim 0.3 \mathrm{~s}$ and $\sim 40 \mathrm{~s}$, with a deficit of bursts of duration $\sim 2 \mathrm{~s}$ dividing the two groups. There is evidence for a duration-spectral hardness correlation in which the short duration bursts are harder than the long bursts (Dezalay et al. 1992; Kouveliotou et al. 1993). Investigations using other instruments, however, have shown that the division of GRBs based on duration is typically energy-dependent (Bromberg et al. 2012) and the $2 \mathrm{~s}$ division found in BATSE is a result of the detector bandpass.

The prompt spectra of gamma-ray bursts have been measured (e.g., Cline et al. (1973); Mazets et al. (1981); Matz et al. (1985); Norris et al. (1986); Band et al. (1993); Dingus et al. (1994); Pelangeon et al. (2008); Frontera et al. (2009); Sakamoto et al. (2011)) over a wide energy range $(<1 \mathrm{keV}$ to several GeV). Many burst spectra are consistent with a power law at high energies, showing little or no attenuation of high energy photons, while others exhibit an exponential cutoff at high energies. GRB spectra observed by BATSE at lower energies usually exhibit increasing energy flux at lower energies, with a break between the low and high energy portions of the spectra usually occurring at approximately 100-300 $\mathrm{keV}$, although the break has been found below a few tens of keV in X-Ray Flashes (XRFs) studied by the HETE-2 experiment (Atteia \& Boer 2011), as well as several examples of breaks existing in the MeV range (e.g.; Briggs et al. 1999; Goldstein et al. 2012). Most, but not all, gamma-ray bursts exhibit significant spectral evolution, usually evolving from hard to soft.

It is because of the many aforementioned reasons that it is appropriate and enlightening to systematically study the spectral properties of GRBs. Earlier spatial studies focused on 
the time-resolved properties of GRB spectra, which were only possible for very bright bursts (Preece et al. 1998; Kaneko et al.2006). However, there is also a need for analyses that derive properties for all possible bursts. Indeed, several studies made use of a partially complete set of spectral analyses of BATSE GRBs (Mallozzi et al. 1995; Band \& Preece 2005) and one published result has been based upon the complete set (Goldstein et al. 2010). The BATSE dataset of GRBs is currently the largest compendium of GRB observations from a single instrument, and as an increasing number of observations are made within the lower-energy bandpass Swift and the higher-energy and broader bandpass of Fermi, the BATSE dataset will be an important bridge between the sub-keV, MeV, and $\mathrm{GeV}$ observations of the prompt emission of GRBs. Furthermore, although the energy band of the currently operating Fermi/GBM completely covers the BATSE energy range, the GBM cannot match the sensitivity and effective area that BATSE detectors possessed. Here, we present the complete set of spectral analyses from which these works were derived, corresponding to the burst selection of the 5B BATSE Burst Catalog (Briggs, et al., in preparation), covering the entire BATSE mission. All catalog files are available from a public archive (HEASARC, http://heasarc.gsfc.nasa.gov/W3Browse/cgro/bat5bgrbsp.html).

We start with a description of the BATSE detectors and calibration in Section 2. This is followed in Section 3 by a description of the methodology used in the production of this catalog, including detector selection, data types used, energy selection and background fitting, and the source selection We then offer a description of the spectral models used in this catalog in Section 4. Finally, in Section 5 we present the spectral analysis methods and results. 


\section{Detectors and Calibration}

The Compton Gamma-Ray Observatory (CGRO) was placed into low Earth orbit $(\sim 400 \mathrm{~km})$ by the space shuttle Atlantis on April 5, 1991. BATSE was one of four experiments on-board the 17 ton satellite. It was an eight-module all-sky detector system designed to study gamma-rays in the energy band of $\sim 10 \mathrm{keV}-20 \mathrm{MeV}$. Each of the eight detector modules were mounted on the corners of CGRO. They consisted of two NaI(T $\ell$ ) scintillation detectors: a Large Area Detector (LAD), optimized for temporal resolution, and a Spectroscopy Detector (SD), optimized for energy resolution. The configuration of the experiment allowed the maximum unobstructed field of view (approximately $2.6 \pi$ steradians) for a low Earth orbit satellite.

Each LAD contained a NaI crystal $\sim 51 \mathrm{~cm}$ in diameter and $\sim 1.3 \mathrm{~cm}$ thick that was uncollimated in the forward hemisphere and passively shielded in the aft hemisphere. The large surface area enabled the collection of a large number of gamma-ray photons as compared to previous orbiting scintillation detectors, thus providing a superior combination of temporal and energy resolution of observed events. The LAD was mounted on the upper part of the module. The planes of the eight LAD faces formed a canonical octahedron when the modules were in their flight configuration on the CGRO. This ensured that each GRB usually illuminated three or four detectors (with a special case where only two

detectors were illuminated). The approximate location of a burst could then be determined by comparing the relative count rates in those detectors that observed the burst (Horack 1991).

The LAD angular energy response is, to first order, a cosine function in $\theta$ at low energies, where $\theta$ is the angle of the GRB from the normal of the LAD crystal. The response is flatter than a cosine function for energies greater than $\sim 300 \mathrm{keV}$ due to decreasing detector efficiency at higher energies. The circular configuration and lack of 
spacecraft interference in the forward hemisphere of each LAD essentially removes any azimuthal dependence of the response function. Hence, the LAD detector response matrices (DRMs) used in this study do not incorporate any azimuthal dependence in the response function. The DRMs are mathematical matrix representations of the detector energy response used to map the observed counts into photons of known energy. Each detector's response is dependent on incident photon energy, the measured detector output energy, and the detector- source angle and the earth-source-spacecraft geometry (Pendleton et al. 1995). For details on the LAD effective area and response, see McNamara et al. (1995) and Laird et al. (2006).

Although most scintillation detectors typically have photomultiplier tubes (PMTs) coupled directly to the crystal, the large surface area of the LADs made this impractical for uniform light collection. The crystal was instead attached to a collection cone that was lined with a highly reflective barium sulfate-based $\left(\mathrm{BaSO}_{4}\right)$ coating. Three PMTs collected the scintillation photons and these signals were summed at each detector. The tubes had a minimum quantum efficiency of $26 \%$ at $410 \mathrm{~nm}$ (Horack 1991). The light collection cone was lined with lead and tin layers providing passive shielding in the rear hemisphere up to $\sim 300-400 \mathrm{keV}$. The outermost tin layer was designed to absorb the $\mathrm{K}$-shell X-rays produced by gamma interactions in the lead. The front of the crystal was covered by a plastic scintillator whose light was collected by two $5 \mathrm{~cm}$ PMTs, the signals of which were also summed at each detector. The purpose of this plastic scintillator, called the Charged Particle Detector (CPD), was to detect particles that entered the LADs. The instrument could be prevented from triggering due to radiation produced by interactions of these particles with the NaI crystal by the detectors' optional coincidence/anti-coincidence circuitry (the instrument operated in anti-coincidence mode for the entire mission). No energy information was available for incident particles; the plastic scintillator was used solely to aid in identification of charged particle events. The threshold energy deposition 
for the charged particle detector was $\sim 500 \mathrm{keV}$.

Each LAD was equipped with a system that controlled the high voltages (gain) of the photomultiplier tubes with minimal intervention from controllers on the ground. The Automatic Gain Control (AGC) algorithm computed and executed adjustments to the PMT high voltages so that a feature in the count spectrum remained in a specified energy channel. The background feature nominally monitored by the AGC was the $511 \mathrm{keV}$ electron-positron annihilation line that is present in the gamma-ray background. The background was calculated as a straight line over a specified range of energy channels and was used to produce a background-subtracted count spectrum. The channel centroid of the $511 \mathrm{keV}$ line was was computed, and if this centroid was different from a specified channel, the high voltage of a single PMT was adjusted to correct the computed value. If the background line drifted too far from the programmed energy channel, the gains of the PMTs were automatically adjusted $\sim 4$ volts higher or lower to move the annihilation line back to the correct energy channel. The PMTs were adjusted cyclically, and the range of voltage adjustment was clamped. If the AGC attempted to change the high voltage to values outside of the specified range, an error was issued. This procedure, which occurred approximately every 5 minutes, ensured that the eight LADs had nearly equal energies in a given pulse-height channel. A sample of the variation in the $511 \mathrm{keV}$ calibration line near the beginning and end of the mission is shown in Figure 1, where it is shown that generally the gain varied by only a fraction of a percent. Furthermore, examples of the observed background spectrum near the start and end of the mission can be found in Figure 2, which displays an enhancement of an activation line at $\sim 191 \mathrm{keV}$ near the end of the mission. Additional details of the energy calibration of the LADs such as the Crab Nebula spectral analysis and energy-PHA relation can be found elsewhere (Band et al. 1992; Pendleton et al. 1994; Preece et al. 1998). 


\section{Method}

During its entire 3323 days of operation (an effective exposure of $\sim 2390$ days in the GRB triggering energy band), BATSE triggered on 2704 GRBs, 2145 of which are presented in this catalog. Bursts that were excluded include those with a low accumulation of count rates or a lack of spectral/temporal coverage. In some cases the data collected onboard the spacecraft would be corrupted during storage or during transmission to ground. For this

reason, many bursts do not have contiguous data and so were not included in the catalog. In a few cases data were available but contained incomplete time history, either through data corruption or count rate truncation from an extreme number of detected counts ( $\sim 5 \times 10^{4}$ counts/energy channel/s above background, where 'energy channel' refers to the channels in the triggering energy band). These bursts were also omitted, as were extremely bright detections where the count rates were so large in each energy channel that caused detector saturation, and in some cases pulse pile-up. In order to provide the most useful analysis to the community, we have attempted to make the method as objective, systematic and uniform as possible. When we deviate from uniformity we indicate the circumstances clearly.

\subsection{Detector Selection}

BATSE employed a total of 16 detectors in pairs of two on each the eight corners of the spacecraft. Each pair comprised a LAD and SD detector. For the purposes of this catalog, we have chosen to use only the LADs because of their much larger effective area, and the fact that the DRMs for the SDs exhibit poor photopeak efficiencies and large off-diagonal DRM elements at energies $>3 \mathrm{MeV}$ (Kaneko 2005). The area of each LAD was $2025 \mathrm{~cm}^{2}$ with a spectral coverage from $\sim 20 \mathrm{keV}$ to $\sim 2 \mathrm{MeV}$ and a peak spectral response at $\sim 50-200 \mathrm{keV}$. To ensure sufficient detector response, only detectors with viewing angles 
to the burst less than $65^{\circ}$ with respect to the LAD normal were included in the analysis. These were selected from the subset of the four brightest detectors for each GRB, since the count rate in the detector is highly dependent on the incident angle. In most cases this resulted in multiple detectors per burst. Rather than performing joint spectral fits of all relevant detectors for a burst, we integrated the count rates over all relevant detectors, while preserving the energy edges, as well as integrating over the detector responses. This method boosts the signal-to-noise and allows the inclusion of many weaker bursts into the catalog. Another benefit of this method is that it helps to minimize the dilution of noise in the signal selection, especially for bursts less than 2 seconds. A trade-off, however, is that there is an increase in systematic uncertainties that may not be completely accounted for in the error propagation of our results. In Appendix D we show the impact of this is minimal when compared to the benefit of improved statistics.

\subsection{Data Types}

The primary data type used in this catalog was the 2.048 second resolution CONT data, which provided semi-continuous count rate and 16-channel spectral coverage during the entire BATSE lifetime. Other BATSE datatypes such as HERB or SHERB data provide a much higher energy resolution (128 and 256 channels respectively) at the cost of time resolution. These datatypes provided pre-burst time resolution on the order of $\sim 300 \mathrm{~s}$, which complicates accurate background subtraction. Additionally, the trigger time resolution can vary depending on the count rate, and in many cases the high temporal resolution and trigger data end before the end of the GRB, further compromising the spectral analysis. Therefore, CONT data are a prime choice for time-integrated spectral fitting since they allow the analysis of any precursor or late-time prompt emission that is

not covered by other data types. Unfortunately, this datatype places all of the emission of 
classically defined short GRBs into a single time bin and at times includes background in the signal selection. The background contamination in this catalog, however, is marginal since most of the short GRBs in this catalog have a high signal-to-noise ratio, and they are only slightly affected by the inclusion of a relative small amount of noise. An example of this is trigger \#206, which is representative of a lower than average intensity short GRB observed by BATSE. The signal-to-noise ratio (SNR) for this GRB is 5.7 using the single $2.048 \mathrm{~s}$ bin in the CONT data, compared to a SNR of 7.6 when selecting the region in the $16 \mathrm{~ms}$ resolution MER data.

The spectral coverage of the CONT data is split into 16 bins, including the noise-prone low-energy channel and high-energy overflow channel, so we used the remaining 14 energy channels for spectral fitting. While this is a coarser energy resolution than some data types such as HERB or SHERB, 14 channels are more than twice the number of free parameters

of the models being fit, so they are statistically sufficient for model fitting and comparison of GRBs (see Appendix A for simulations confirming the accuracy of CONT data as compared to HERB data). When CONT data were not available (usually due to data corruption), the associated MER data were used if possible. The native time resolution of MER data was $16 \mathrm{~ms}$ but only started at trigger time and extended to less than $200 \mathrm{~s}$ after trigger. The energy resolution is the same as CONT data, so the count rates were binned to 2.048 $\mathrm{s}$ in order to be compatible with CONT data. MER data were used only if a background model could be fit to the post-burst background and extrapolated through the duration of the burst. MER data was used for only 15 GRBs in this catalog.

\section{3. $\quad$ Energy Selection and Background Fitting}

With the optimum subset of detectors selected, the best time and energy ranges are then chosen to fit the data. From the available energy channels in the LADs we select 
channels 1-14, corresponding to energies between $\sim 30 \mathrm{keV}$ and $\sim 1.8 \mathrm{MeV}$. This selection is performed to exclude the high-energy overflow channel and the low-energy channel where the instrument response is poor and the background is high. With the resulting time series, we select long pre- and post-burst background intervals to sufficiently model the background and fit a single polynomial (up to $4^{\text {th }}$ order) to each energy channel in the background selection. For each detector the time selection and polynomial order are varied until the $\chi^{2}$ statistic map over all energy channels is minimized resulting in an adequate background fit. Typically a first- or second-order polynomial was fit to the background, since BATSE generally had a fairly stable background due to its constant inertial-pointing. Shown in Figure 3 is an example of the regions selected as background for a GRB. Only the bursts using MER data that had a background that could be sufficiently fit with a first order polynomial were included in the catalog, since only post- burst background was available for those bursts with only MER data.

\subsection{Source Selection}

Once the background count rates are determined, we subtract the background, convert to counts, and calculate the signal-to-noise ratio (SNR) in the $20 \mathrm{keV}-2 \mathrm{MeV}$ band for each time bin. Only the bins that had a SNR greater or equal to 3.5 sigma were selected as signal. This criterion ensures that there is adequate signal to successfully perform a spectral fit and constrain the parameters of the fit. This does however eliminate some faint bursts from the catalog sample (i.e., those with no time bins with signal above 3.5 sigma). In addition, this strict cut was performed out of the need to provide an objective catalog, so we note that it is possible that not all signal from a burst was selected. However, most of the signal below 3.5 sigma is likely indiscernible from the background fluctuations, so a spectral analysis including those bins would likely only increase the uncertainty in the 
measurements without improving the measurement. This selection is referred to as the "fluence" selection, since it is a time-integrated selection, and is representative of the fluence over the duration of the burst as defined by the count rate bins that are above the SNR threshold. The other selection performed is based on a 2.048 s peak photon flux, namely selecting the single time-history bin of signal with the highest background-subtracted count rate, (i.e., the most intense part of the burst). We define the accumulation time as the total amount of signal selected using the 3.5 sigma SNR criterion, which ignores quiescent periods during the burst. Figure 4 shows the distribution of accumulation time based on the signal-to-noise selection criteria. The accumulation time reported is similar to the observed emission time of the burst, excluding quiescent periods, similar to previous studies by Mitrofanov et al. (1999). As a result of the datatype used in this catalog, the accumulation time is quantized by multiples of $2.048 \mathrm{~s}$, thereby eliminating evidence of bimodality of the accumulation time. Figure 4 also includes the comparisons of the model photon fluence and photon flux compared to the accumulation time. Note that both comparisons contain two distinct regions associated with short and long GRBs. While there appears to be a very clear correlation between the photon fluence and the accumulation time, there is little correlation between the burst-averaged photon flux and the accumulation time.

\section{Models}

We chose five spectral models to fit the spectra of GRBs in our selection sample. These models include a single power law (PL), Band's GRB function (BAND), an exponential cut-off power-law (COMP), a smoothly-connected broken power law (SBPL), and a $\log _{10}$ Gaussian (GLOGE). All models are formulated in units of photon flux with energy (E) in $\mathrm{keV}$ and multiplied by a normalization constant $\mathrm{A}\left(\mathrm{ph} \mathrm{s}^{-1} \mathrm{~cm}^{-2} \mathrm{keV}^{-1}\right)$. Below we detail each model and its features. 


\subsection{Power-Law Model}

An obvious first model choice ubiquitous in astrophysical spectra, is the simple single power law with two free parameters,

$$
f_{P L}(E)=A\left(\frac{E}{E_{p i v}}\right)^{\lambda}
$$

where $A$ is the amplitude and $\lambda$ is the spectral index. The pivot energy $\left(E_{\text {piv }}\right)$ normalizes the model to the energy range under inspection and helps reduce cross-correlation of other parameters. In all cases in this catalog, $E_{\text {piv }}$ is held fixed at $100 \mathrm{keV}$. While most GRBs exhibit a spectral break in the BATSE passband, some weak GRBs are too weak to adequately constrain this break in the fits and therefore we chose to fit these with the PL model.

\subsection{Band's GRB function}

Band's GRB function (Band et al. 1993) has become a standard spectral form for fitting GRB spectra, and therefore we include it in our analysis::

$$
f_{B A N D}(E)=A \begin{cases}\left(\frac{E}{100 \mathrm{keV}}\right)^{\alpha} \exp \left[-\frac{(\alpha+2) E}{E_{\text {peak }}}\right] & E \geq \frac{(\alpha-\beta) E_{\text {peak }}}{\alpha+2} \\ \left(\frac{E}{100 \mathrm{keV}}\right)^{\beta} \exp (\beta-\alpha)\left[\frac{(\alpha-\beta) E_{\text {peak }}}{100 \mathrm{keV}(\alpha+2)}\right]^{\alpha-\beta} & E<\frac{(\alpha-\beta) E_{\text {peak }}}{\alpha+2}\end{cases}
$$

The four free parameters are the amplitude, $A$, the low and high energy spectral indices, $\alpha$ and $\beta$, respectively, and the $\nu F_{\nu}$ peak energy, $E_{\text {peak }}$. This function is essentially a smoothly broken power law with a curvature defined by its spectral indices. The low-energy index spectrum asymptotically becomes a power law. 


\subsection{Comptonized Model}

This model is an exponentially cutoff power-law which is a subset of the Band function in the limit that $\beta \rightarrow-\infty$ :

$$
f_{C O M P}(E)=A\left(\frac{E}{E_{\text {piv }}}\right)^{\alpha} \exp \left[-\frac{(\alpha+2) E}{E_{\text {peak }}}\right]
$$

The three free parameters are the amplitude $A$, the low energy spectral index $\alpha$ and $E_{\text {peak }}$. $E_{\text {piv }}$ is again fixed to $100 \mathrm{keV}$, as for the power law model.

\subsection{Smoothly Broken Power-Law}

Another model that we consider in this catalog is a broken power-law characterized by one break with flexible curvature able to fit spectra with both sharp and smooth transitions between the low and high energy spectra. This model, first published in Ryde (1999) where the logarithmic derivative of the photon flux is a continuous hyperbolic tangent, has been re-parametrized (Kaneko et al. 2006) as:

$$
f_{S B P L}(E)=A\left(\frac{E}{E_{p i v}}\right)^{b} 10^{\left(a-a_{p i v}\right)}
$$

where

$$
\begin{aligned}
& a=m \Delta \ln \left(\frac{e^{q}+e^{-q}}{2}\right), \quad a_{p i v}=m \Delta \ln \left(\frac{e^{q_{p i v}}+e^{-q_{p i v}}}{2}\right) \\
& q=\frac{\log \left(E / E_{b}\right)}{\Delta}, \quad q_{p i v}=\frac{\log \left(E_{p i v} / E\right)}{\Delta}, \\
& m=\frac{\lambda_{2}-\lambda_{1}}{2}, \quad b=\frac{\lambda_{1}+\lambda_{2}}{2} .
\end{aligned}
$$

In the above relations, the low- and high-energy power law indices are $\lambda_{1}$ and $\lambda_{2}$ respectively, $E_{b}$ is the break energy in $\mathrm{keV}$, and $\Delta$ is the break scale given in decades of energy. The 
break scale is independent and not coupled to the power law indices as it is with the Band function, and as such represents an additional degree of freedom. However, Kaneko et al. (2006) found that an appropriate value for $\Delta$ for GRB spectra is 0.3 , therefore we fix $\Delta$ at this value.

\section{5. $\log _{10}$ Gaussian}

The final model that we consider in this catalog is a Gaussian parametrized with logarithmic energies, or GLOGE. The photon model is represented by

$$
f_{G L O G E}(E)=\frac{A}{\sqrt{2 \pi} s} \exp \left[-\frac{1}{2}\left(\frac{\log _{10} E-\log _{10} E_{c e n}}{s}\right)^{2}\right],
$$

where $E_{c e n}$ is the centroid energy in $\mathrm{keV}$ and $s$ is the standard deviation at $E_{c e n}$ in decades of $\mathrm{keV}$. This model is identical to the log-parabolic function that is common in investigating BL Lac spectra, and has recently been used to study GRB spectra (Massaro et al. 2010).

\section{Data Analysis \& Results}

To study the spectra resulting from the BATSE detectors, a method must be established to associate the energy deposited in the detectors to the energy of the detected photons. This association is dependent on effective area and the angle of the detector to the incoming photons. To do this, detector response matrices (DRMs) are used to convert the photon energies into detector channel energies. The DRM is a mathematical model of the deposition of photon energy in the crystal - a photon that interacts by the photoelectric effect will deposit $100 \%$ of its energy, subject to resolution broadening, while a photon that interacts by a single Compton scatter may deposit only a portion of its energy. The exception to this is when a photon carrying the energy of the iodine K-shell escapes the crystal. The energy calibration determines the energy boundaries of the energy 
deposition channels. The response matrices for all GRBs in the catalog were made using DRM_LAD_GEN v2.07 of the response generator for BATSE Pendleton et al. (1995).

The spectral analysis of all bursts was performed using RMfit, version 3.4rc1. RMfit employs a modified, forward-folding Levenberg-Marquardt algorithm for spectral fitting. The Castor C-Statistic, which is a modified log-likelihood statistic based on the Cash parametrization (Cash 1979) is used in the model-fitting process as a figure of merit to be minimized. This statistic is preferable over the more traditional $\chi^{2}$ statistic minimization because of the non-Gaussian counting statistics present when studying dim GRBs. Although it is advantageous to perform the spectral fitting using C-Stat, the statistic provides no estimation of the goodness-of-fit, since there exists no standard probability distribution for likelihood statistics. For this reason, we also calculate $\chi^{2}$ for each spectral fit performed by minimizing C-Stat. This allows an estimation of the goodness-of-fit of a function to the data even though $\chi^{2}$ was not minimized (see Appendix $\mathrm{C}$ for simulations). This also allows for easy comparison between nested models.

We fit the five functions described in Section 4 to the spectra of each burst. The BAND and COMP functions are parametrized with $E_{\text {peak }}$, the peak in the power density spectrum, while the SBPL is parametrized with the break energy, $E_{b r e a k}$, and the GLOGE model is parametrized with the centroid energy $E_{c e n}$. We choose to fit these five different functions because the measurable spectrum of GRBs is dependent on intensity, as is shown in Figure 5. Observably less intense bursts provide less data to support a large number of parameters. This allows us to determine why, in many situations, a particular empirical function provides a poor fit, while in other cases it provides an accurate fit. For example, the energy spectra of GRBs are normally well fit by two smoothly joined power laws. For particularly bright GRBs, the BAND and SBPL functions are typically an accurate description of the spectrum, while for weaker bursts the COMP or GLOGE 
function is most acceptable. Bursts that have signal significance on the order of the background fluctuations do not have a detectable distinctive break in their spectrum and so the power law is the most acceptable function. Although weaker GRBs do not statistically prefer a model with more parameters, it is instructive to study the parameters of even the weaker bursts. In addition, the actual physical GRB processes can have an effect on the spectra and different empirical models may fit certain bursts better than others. The spectral results, including the best fit spectral parameters and the photon model, are stored in files following the FITS standard similar to those described in the Appendix of Goldstein et al. (2012) and are hosted as a public data archive on HEASARC (http://heasarc.gsfc.nasa.gov/W3Browse/cgro/bat5bgrbsp.html). Many of the important spectral quantities are also available in tables available in the electronic version of this catalog following the formats listed in Tables 1.5.

When inspecting the distribution of the parameters for the fitted models, we first define a data cut based on the goodness-of-fit. We require that the $\chi^{2}$ statistic for the fit to be within the $3 \sigma$ expected region for the $\chi^{2}$ distribution of the given degrees of freedom, and we define a subset of each parameter distribution of this data cut as GOOD if the error is within certain limits. Following Kaneko et al. (2006), for the low-energy power law indices, we consider GOOD values to have errors less than 0.4, and for high-energy power law indices we consider GOOD values to have errors less than 1.0. For all other parameters we consider a GOOD value to have a relative error of 0.4 or better. The motivation for this is to show well-constrained parameter values, rather than basing possible interpretations on parameters that are poorly constrained.

In addition, we define a BEST sample where we compare the goodness-of-fit of all spectral models for each burst and select the most preferred model based on the difference in $\chi^{2}$ per degree of freedom. The criterion for accepting a model with a single additional 
parameter is a change in $\chi^{2}$ of at least 6 since the probability for achieving this difference is $\sim 0.01$. The parameter distributions are then populated with the spectral parameters from the BEST spectral fits.

\subsection{Fluence Spectra}

The time-integrated fluence distributions are estimated over the duration of the observed emission, where the observed emission is defined as $3.5 \sigma$ over the estimated background in the 20-2000 keV energy range. It should be noted that the following distributions do not take into account any spectral evolution that may exist within bursts. The low-energy indices, as shown in Figure 6, distribute about a -1 power law typical of most GRBs. Accounting for parameter uncertainty, up to $14 \%$ of the GOOD low-energy indices violate the $-2 / 3$ synchrotron "line-of-death" (Preece et al.|2002), while an additional $57 \%$ of the indices violate the $-3 / 2$ synchrotron cooling limit. The high-energy indices in Figure 7 peak at a slope slightly steeper than -2 and have a long tail toward steeper indices. Note that the large number of unconstrained (or very steep) high-energy indices in the distribution of all high-energy index values indicates that a large number of GRBs are better fit by the COMP model, which is equivalent to a BAND function with a high-energy index of $-\infty$. The comparison of the simple power law index to the low- and high-energy indices makes evident that the simple power law index is averaged over the break energy, resulting in a index that is on average steeper than the low-energy index yet shallower than the high-energy index. We also show in Figure $7(\mathrm{~d})$ the difference between the time-integrated low- and high-energy spectral indices, $\Delta S=(\alpha-\beta)$. This quantity is useful since the synchrotron shock model makes predictions of this value in a number of cases (Preece et al. 2002). The peak of this distribution is at $\sim 1.5$, which indicates a peak electron energy spectral index of $\sim 4$ assuming the traditional synchrotron shock 
model (Sari, Narayan, \& Piran 1996). Note that the large overflow of $\Delta S$ at $\sim 10$ for the BAND model is due to the extremely steep slope of $\beta$, where the high-energy spectrum approximates an exponential cut-off.

Shown in Figure 8 are the distributions for the centroid energy, $E_{c e n}$ in $\mathrm{keV}$, and the full-width at half maximum, FWHM in $\log \mathrm{keV}$, of the GLOGE function. The $E_{c e n}$ distribution peaks at $\sim 10 \mathrm{keV}$ and covers two orders of magnitude while the FWHM peaks at $\sim 1.22$ and covers less than one order of magnitude. Note that the curvature of the GLOGE function can be well constrained by the data even when the centroid of the GLOGE fit is below the BATSE bandpass. As an example of this behavior, $10^{4}$ synthetic GLOGE spectra were generated with $E_{c e n}=8.7 \mathrm{keV}$, the spectra were folded through a CONT DRM, and Poisson fluctuations were added to the total observed counts as well as the background counts. Figure 9 shows the resulting distribution of $E_{c e n}$ from performing spectral fits on the synthetic spectra. As can be seen, the data has no problem in constraining a centroid value below $20 \mathrm{keV}$.

In Figure 10, we show the distributions for the break energy, $E_{b r e a k}$ and the peak of the power density spectrum, $E_{\text {peak }}$. $E_{\text {break }}$ is the energy at which the low- and high-energy power laws are joined, which is not necessarily representative of the $E_{\text {peak }}$. As discussed in Kaneko et al. (2006), although the SBPL is parametrized with $E_{\text {break }}$, the $E_{\text {peak }}$ can be derived from the functional form. Note that we have calculated the $E_{\text {peak }}$ for all bursts with low-energy index shallower than -2 and high-energy index steeper than -2 , and we have used the covariance matrix to formally propagate and calculate the errors on the derived $E_{\text {peak }}$. Similarly, we show in Appendix B, the method to derive $E_{\text {peak }}$ from the GLOGE model. The $E_{\text {break }}$ for the SBPL peaks near $200 \mathrm{keV}$, but has a significant tail towards lower energies. Comparatively, the peak for the BAND distribution is also near $200 \mathrm{keV}$, but less broad and there is no apparent evidence for a low-energy tail. The $E_{\text {peak }}$ distributions for 
all functions generally peak around $200 \mathrm{keV}$, except the derived $E_{\text {peak }}$ for GLOGE, which peaks closer to $100 \mathrm{keV}$. Note that the lower $E_{\text {peak }}$ resulting from GLOGE spectral fits are likely due to the $E_{c e n}$ peaking below the BATSE bandpass. All $E_{\text {peak }}$ distribution cover about two orders of magnitude, which is consistent with previous findings (Mallozzi et al. 1995; Lloyd et al. 2000). The peak and overall distribution of $E_{\text {peak }}$ is similar to that found by bursts observed by the GBM NaI and BGO detectors (Goldstein et al. 2012), which had a much smaller collecting area but larger bandwidth. This would seem to indicate that it is unlikely for there to be a hidden population undiscovered by either instrument within the $\sim 20 \mathrm{keV}-2 \mathrm{MeV}$ range. Additionally, the value of $E_{\text {peak }}$ can strongly affect the measurement of the low-energy index of the spectrum, as shown in Figure 11. A general trend appears to show that lower $E_{\text {peak }}$ values tend to increase the uncertainty in the measurement of the low-energy index, mostly due to the fact that a spectrum with a low $E_{\text {peak }}$ will exhibit most of its curvature near the lower end of the instrument bandpass. In many cases, if the low-energy index is found to be reasonably steep $(\lesssim-1)$, the uncertainty of the index is minimized even if $E_{\text {peak }}$ is low.

It is of interest to study the difference in the value of $E_{\text {peak }}$ between the BAND and COMP functions. The relative deviation between the two values can be calculated from a statistic based on the difference between the values, taking into account their errors. This statistic can be calculated by

$$
\Delta S=\frac{\left|E_{\text {peak }}^{C}-E_{\text {peak }}^{B}\right|}{\sigma_{E_{\text {peak }}}^{C}+\sigma_{E_{\text {peak }}}^{B}}
$$

where $\mathrm{C}$ and $\mathrm{B}$ indicate the COMP and BAND values respectively. This statistic has a value of unity when the deviation between the $E_{\text {peak }}$ values exactly matches the sum of the $1 \sigma$ errors. A value less than one indicates the $E_{\text {peak }}$ values are similar within errors, and a value greater than one indicates that the $E_{\text {peak }}$ values are not within errors of each other. Figure 12 depicts the distribution of the statistic and roughly $33 \%$ of the BAND and COMP $E_{\text {peak }}$ values are found to be outside of the combined errors. This indicates that, 
although COMP is a special case of BAND, a significant fraction of the $E_{\text {peak }}$ values can vary by more than $1 \sigma$ based on which model is chosen.

The distributions for the time-integrated photon flux and energy flux are shown in Figure 13. The photon flux peaks at $\sim 0.4-0.7$ photons $\mathrm{cm}^{-2} \mathrm{~s}^{-1}$, and the energy flux peaks at $8 \times 10^{-8}$ ergs $\mathrm{cm}^{-2} \mathrm{~s}^{-1}$ in the $20-2000 \mathrm{keV}$ band. Similarly in Figure 14 , the distributions for the photon fluence and energy fluence are depicted. The plots for the photon fluence appear to contain evidence of the duration bimodality of GRBs, and has discriminant peaks at $\sim 1$ and 20 photons $\mathrm{cm}^{-2}$. The energy fluence in the full BATSE bandpass peaks at $\sim 2 \times 10^{-6} \mathrm{erg}^{-2}$. The brightest GRB contained in this catalog based on time-averaged photon flux is GRB 931206 (trigger \#2680) with a flux of $>32 \mathrm{ph} \mathrm{s}^{-1} \mathrm{~cm}^{-2}$ and the burst with the largest average energy flux is GRB 991216 (trigger \#7906) with an energy flux of $\sim 3.8 \times 10^{-6} \mathrm{erg} \mathrm{s}^{-1} \mathrm{~cm}^{-2}$. The most fluent burst (although not the longest duration) in the catalog is GRB 990104B (trigger \#7301) with a photon fluence of $>1500 \mathrm{ph} \mathrm{cm}^{-2}$ and an energy fluence of $>3.8 \times 10^{-4} \mathrm{erg}_{\mathrm{cm}^{-2}}$.

\subsection{Peak Flux Spectra}

The following peak flux spectral distributions have been produced by fitting the GRB spectra over the $2048 \mathrm{~ms}$ peak count flux. Note that short bursts with durations less than $2048 \mathrm{~ms}$ will constitute a single bin in the lightcurve and it is that single bin that is selected for analysis. The low-energy indices, as shown in Figure 15 distribute about a -1 power law typical of most GRBs. Accounting for the parameter uncertainty, up to $28 \%$ of the GOOD low-energy indices violate the $-2 / 3$ synchrotron "line-of-death", while an additional $55 \%$ of the indices violate the $-3 / 2$ synchrotron cooling limit. The high-energy indices in Figure 16 peak at a slope of $\sim-2$ and have a long tail toward steeper indices. As shown with the fluence spectra, the PL index serves as an average between low- and high-energy indices for 
the BAND and SBPL functions. Shown in Figure $16(\mathrm{~d})$ is the $\Delta S$ distribution for the peak flux spectra. Similar to the fluence spectra, this distribution peaks at $\sim 1.5$, which implies a median electron energy spectral index of $\sim 4$ assuming the synchrotron shock model. As was the case with the fluence spectra, the overflow of $\Delta S$ at $\sim 10$ for the BAND model is due to the extremely steep slope of $\beta$, where the high-energy spectrum approximates an exponential cut-off.

As shown in Figure 17, the GLOGE $E_{c e n}$, covering two orders of magnitude, is shifted to peak at $\sim 30 \mathrm{keV}$, while the FWHM distribution peaks at $\sim 1.22$ in contrast to the fluence spectral results. In Figure 18 , we show the distributions for $E_{\text {break }}$ and $E_{\text {peak }}$. As was evident from the fluence spectra, the $E_{\text {break }}$ from the SBPL fits appears to peak at $200 \mathrm{keV}$ with a low-energy tail, meanwhile the $E_{\text {break }}$ from BAND is harder and peaks at about $300 \mathrm{keV}$. Consistent with previous findings (Preece et al. 1998; Kaneko et al. 2006), the $E_{\text {peak }}$ distributions for all models peak around $200 \mathrm{keV}$, except for the derived $E_{\text {peak }}$ from GLOGE which peaks at slightly more than $100 \mathrm{keV}$, and covers over two orders of magnitude. We calculate and show the $\Delta E_{\text {peak }}$ statistic in Figure 19 and roughly $24 \%$ of the BAND and COMP $E_{\text {peak }}$ values are outside the combined errors.

The distributions for the peak photon flux and energy flux are shown in Figure 20. The photon flux peaks around 1.5 photons $\mathrm{cm}^{-2} \mathrm{~s}^{-1}$, and the energy flux peaks at $2 \times 10^{-7}$ ergs $\mathrm{cm}^{-2} \mathrm{~s}^{-1}$ in the $20-2000 \mathrm{keV}$ band. The GRB with the brightest peak photon flux is GRB 931206 (trigger \#2680) at $>350 \mathrm{ph} \mathrm{s}^{-1} \mathrm{~cm}^{-2}$. This GRB is also the brightest GRB in terms of time-averaged photon flux. Similar to the time-averaged energy flux, the burst with highest peak energy flux is GRB 991216 (trigger \#7906) at $>5.7 \times 10^{-5} \mathrm{ergs} \mathrm{s}^{-1} \mathrm{~cm}^{-2}$.

When studying the two types of spectra in this catalog, it is instructive to study the similarities and differences between the resulting parameters. Plotted in Figure 21 are the low-energy indices, high-energy indices, and $E_{\text {peak }}$ energies of the peak flux spectra as 
a function of the corresponding parameters from the fluence spectra. Most of the peak flux spectral parameters correlate with the fluence spectral parameters on the order of unity. There are particular regions in each plot where outliers exist, and these areas are indications that either the GRB spectrum is poorly sampled or there exists significant spectral evolution in the fluence measurement of the spectrum that skews the fluence

spectral values. Examples of the former case are when the low-energy index is atypically shallow $(\gtrsim-0.5)$ or the high-energy index is steeper than average $(\lesssim-3)$. An example where spectral evolution may skew the correlation between the two types of spectra is apparent in the comparison of $E_{\text {peak }}$. Here, it is likely that a fluence spectrum covering significant spectral evolution will produce a lower energy $E_{\text {peak }}$ than is measured when inspecting the peak flux spectrum. Additionally, a Kolmogorov-Smirnov test was performed between each fluence and peak flux parameter, and the results are listed in Table 6. From these tests, it appears that only the distributions of the high-energy power law index for both the BAND and SBPL are significantly similar between the fluence and peak flux spectra.

\subsection{The BEST Sample}

The BEST parameter sample produces the best estimate of the observed properties of GRBs. By using model comparison, the most preferred model is selected, and the parameters are inspected for that model. The models contained herein and in most GRB spectral analyses are empirical models, based only on the data received; therefore the data from different GRBs tend to support different models. Perhaps it will be possible to determine the physics of the emission process by investigating the tendencies of the data to support a particular model over others. It is this motivation, as well as the motivation to provide a sample that contains the best picture of the global properties of the data, that 
prompted our investigation of the BEST sample.

In Table 7 we present the composition of models for the BEST samples. From this table, it is apparent that the spectral data from BATSE strongly favors the COMP model over the others in nearly half of all GRBs. The BAND and SBPL are favored by a relative few GRBs in the catalog, possibly because of the limited spectral resolution of the data. Table 8 lists the sample mean and and standard deviations comparing the GOOD fluence and peak flux spectra as well as the BEST fluence and peak flux spectra. In figures 22 and 23 the same error cuts used in the GOOD samples were also used for the BEST parameters. Note that the PL index is statistically an averaging of the low- and high-energy power laws; due to the fact that BATSE spectral responses have a peak effective area at lower energies ( $70 \mathrm{keV}$ ), we have included PL indices into the BEST low-energy index distribution. Although in a number of cases the PL model is statistically preferred over the other models in this catalog, the spectral shape represented by the PL is inherently different from the shape of the other models. Therefore, the PL index is not necessarily representative of either the low- or high-energy indices from the other models. In Figures 22(a) and 23(a) we show where the distribution of PL indices exists relative to the alpha and beta distributions. The PL index represents $23 \%$ of the BEST fluence alpha distribution and $21 \%$ of the BEST peak flux alpha distribution. The fluence spectra, on the whole, have a steeper measured alpha and shallower beta than the peak flux spectra. The alpha distribution for the fluence spectra peaks at slightly steeper than -1 , while the peak flux low-energy spectral index peaks at slightly shallower than -1 . Conversely, the beta distribution for the fluence spectra peaks at -2.1 and the peak flux high-energy spectral index peaks at -2.4 .

The $E_{\text {peak }}$ and $E_{\text {break }}$ distributions are similar between the two spectra, however. The fluence spectra $E_{\text {peak }}$ peaks at $\sim 200 \mathrm{keV}$ as does the peak flux spectra $E_{\text {peak }}$ and the $E_{\text {break }}$ peaks at more than $200 \mathrm{keV}$. Note that the $E_{\text {break }}$ distributions are significantly smaller in 
comparison to the $E_{\text {peak }}$ distributions, especially for the fluence spectra, simply because the errors are not as well constrained for $E_{\text {break }}$. As is shown in Figures 22(c) and 23(c) respectively, the fluence spectra photon flux peaks at $0.75_{-0.37}^{+1.09}$ photons $\mathrm{cm}^{-2} \mathrm{~s}^{-1}$ and the peak flux photon flux peaks at $1.61_{-1.02}^{+4.32}$ photons $\mathrm{cm}^{-2} \mathrm{~s}^{-1}$. Comparatively, the fluence energy flux peaks at $\left(1.40_{-0.71}^{+2.49}\right) \times 10^{-7} \mathrm{erg} \mathrm{cm}^{-2} \mathrm{~s}^{-1}$, and the peak flux energy flux peaks at $\left(3.44_{-2.09}^{+11.5}\right) \times 10^{-7} \mathrm{erg} \mathrm{cm}^{-2} \mathrm{~s}^{-1}$, as is shown in Figures 22(d) and 23(d).

To aid in the study of the systematics of the parameter estimation, as well as to garner the effect statistics has on the fitting process, we investigate the behavior of the parameter values as a function of the photon fluence and peak photon flux for the fluence and peak flux BEST spectra, respectively. These distributions are shown in Figures 24 and 25. When fitting the time-integrated spectrum of a burst, we find the low- and high-energy indices trend toward steeper values for exceedingly more fluent spectra. These figures show that the simple PL index trends from shallow value of $\sim-1.3$ to a steeper value of $\sim-2.3$. The low-energy index for a spectrum with curvature tends to exhibit an unusually shallow value of $\sim-0.4$ for extremely low fluence spectra, and steepens to $\sim-1.5$. Similarly, the high-energy index trends from $\sim-1.6$ at low fluence to $\sim-3$ at high fluence, although this is complicated by unusually steep and poorly constrained indices that indicate that an exponential cutoff results in a more reliable spectral fit. There are a number of cases where the COMP spectral index is unusually steep $(\sim-2)$, which may indicate that the GRB is particularly soft and that $E_{\text {peak }}$ exists sufficiently below the detector bandpass. In these cases, BATSE is only observing the high-energy power law of the GRB, which may display an exponential cutoff similar to other GRBs at higher energies. Note that in this situation, the measured $E_{\text {peak }}$ will not be the true peak of the $\nu F_{\nu}$ spectrum, since the true $E_{\text {peak }}$ will exist below $20 \mathrm{keV}$. In other cases, the high-energy power law may not be cutoff and is well-modeled by a PL model, which could be indicated by high flux GRBs with index steeper than -2 . 
Additionally, to test if the trend seen in the low-energy index versus photon fluence is a product purely of unknown systematics issues from the spectral fitting of weak GRBs, we performed five sets of 10000 simulations of the BAND model by using five different $E_{\text {peak }}$ values and a low energy index of -0.5 and a high energy index of -2.6 . Different photon fluences were also simulated by changing the simulated live time of the spectra using 11 equally spaced values in logarithmic space spanning from $0.128-1049 \mathrm{~s}$. The simulations were then fit and the mean and uncertainty of the resulting parameters from each set of simulations was calculated, and the BAND alpha-photon fluence figure is shown in Figure 26. From these simulations we deduce that the trend seen in the catalog does not appear to be from systematic effects due to the low fluence of GRBs. In fact, these simulations show that on the average, a GRB with a fluence $<10$ photons $\mathrm{cm}^{-2}$ will tend to under-predict the true value of the BAND alpha, which would show a positive trend if there was only this one systematic effect. The other finding from these simulations is that only for $E_{\text {peak }} \gtrsim 100$ $\mathrm{keV}$ can the BAND alpha value be reliably found even out to $\sim 1000$ photons $\mathrm{cm}^{-2}$.

When inspecting the $E_{\text {peak }}$ as a function of photon fluence, a trend is much less apparent. If a burst is assumed to have significant spectral evolution, then obviously the $E_{\text {peak }}$ will change values through the time history of the burst, typically following the traditional hard-to-soft energy evolution. For this reason, spectra that are integrated over increasingly longer time intervals will tend to suppress the highest energy of $E_{\text {peak }}$ within the burst, so a general decrease in $E_{\text {peak }}$ is expected with longer integration times. However, the photon fluence convolves the integration time with the photon flux so that an intense burst with a short duration may have approximately the same fluence as a much longer but less intense burst, albeit in a higher $E_{\text {peak }}$. This causes significant broadening to the decreasing trend as shown in Figure 24(c), The distribution of parameters as a function of the peak photon flux show similar trends, although due to the smaller statistics of the shorter integration time, the trends are much more dispersed. The distributions shown in 
Figure 25 are more susceptible to uncertainty because of the generally smaller statistics involved in the study of the peak flux of the GRB, except in some cases where the peak photon flux is similar to the photon fluence. Ignoring the regions where the parameters are poorly constrained, another trend emerges from the low-energy indices; they appear to become slightly more shallow as the photon flux increases. The high-energy indices, however, appear to be unaffected by the photon flux, except those that are unusually steep and indicate that an exponential cutoff may be preferred. Additionally, an obvious trend emerges from all Figures in 24 and 25: GRBs with lower photon flux and photon fluence are more likely to be best fit by a simple power law, and brighter GRBs with higher photon flux or fluence will typically be best fit by the more complex BAND or SBPL models. This trend displays a model- dependent analog to Figure 5, which shows that the preferred model complexity is correlated to the amount of data present from the burst.

\subsection{Comparisons to Previous Results}

A comparison between different catalogs can be instructive, especially when noting the difference in detector geometry, sensitivity, and bandpass. For example, the Fermi/GBM Spectral Catalog (Goldstein et al. 2012) contains spectral information of many GRBs that were observed by detectors with a total collecting area that is $\sim 1 / 8$ of BATSE, but with a larger bandpass $(\sim 8 \mathrm{keV}-40 \mathrm{MeV})$ and increased high-energy sensitivity. In general, there are no large discrepancies between the two catalogs, although there are subtle differences between the parameter distributions. Table 9 displays a comparison of the sample mean and standard deviations of the GBM spectral catalog and this catalog. The GBM spectral catalog contains, on average, shallower low- and high-energy power law indices when compared to this catalog. The differences are a few tenths of an index, and the distributions in the GBM catalog are not as wide, most likely due to a much smaller sample of GRBs. 
The average $E_{\text {peak }}$ in this catalog is slightly softer and the average $E_{\text {break }}$ is slightly harder than was found from the GBM bursts. As expected, the photon flux distribution for BATSE is shifted to smaller fluxes due to the much larger collecting area of BATSE, enabling the detection and observation of weaker bursts. Similarly, the energy flux distribution for GBM is shifted to larger fluxes due to its increased high-energy sensitivity from the BGO detectors.

Additionally, when comparing our results to the BATSE catalog of bright GRBs (Kaneko et al. 2006), there are some marked differences as well, although many of these arise because the Kaneko et al. (2006) sample studied only the 350 brightest BATSE GRBs. We investigated the sample mean of the time-integrated spectra for comparison (see Table 9) and found that the average low-energy index and high-energy index on average was slightly steeper when compared to Kaneko et al. (2006). The time-averaged $E_{\text {peak }}$ in this catalog, however, is on average softer, most likely due to the fact that included all bursts, not just the brightest bursts detected. This also affects the $E_{b r e a k}$, which is also found to be slightly softer in this catalog; as expected the Kaneko et al. (2006) catalog reports larger average photon and energy fluxes.

\section{Summary}

BATSE provided a uniquely large, homogenous sample of GRB spectra over the 20 keV $-2 \mathrm{MeV}$ energy band. The broad energy range and long operational life of BATSE translates to currently the largest and most comprehensive collection of GRB temporal and spectral properties. The distributions contained here are similar to those shown by previous studies, yet contain differences that display the usefulness of studying GRBs over different energy bands and sensitivities. We have shown in many cases that the fitted spectrum of a GRB depends in large part on its intensity as well as the detector sensitivity. This 
observation implies that weak GRBs may have the same inherent spectrum as their more intense counterparts, yet we are unable to accurately determine their spectrum, reinforcing the importance of comparing the spectral parameter distributions from different acceptable models.

In conclusion, we have presented a systematic analysis of 2145 GRBs detected with BATSE during its entire period of operation. This catalog contains five basic photon model fits to each burst, using two different selection criteria to produce over 19000 spectra and facilitate an accurate estimate of the spectral properties of these GRBs. We have described subsets of the full results in the form of data cuts based on parameter uncertainty, as well as employing model comparison techniques to select the most statistically preferred model for each GRB. The analysis of each GRB was performed as objectively as possible, in an

attempt to minimize biased systematic errors inherent in subjective analysis. The methods we have described treat all bursts equally, and we have presented the ensemble of observed spectral properties of BATSE GRBs. This catalog represents the largest sample of GRBs to date, and constitutes a wide array of GRB spectral properties. Certainly there are avenues of investigation that require more detailed work and analysis or perhaps a different methodology. This catalog should be treated as a starting point for future research on interesting bursts and ideas. As has been the case in previous GRB spectral catalogs, we hope this catalog will be of great assistance and importance to the search for the physical properties of GRBs and other related studies.

\section{Acknowledgments}

AG acknowledges the support of the Graduate Student Researchers Program funded by NASA. 


\section{Appendix A}

\section{Comparison of 16-Channel CONT Spectra to 128-Channel HERB Spectra}

The CONT data used in this catalog contains a total of 16 spectral channels (14 usable) over a broad $\sim 20-2000 \mathrm{keV}$ energy range. To ensure that there is not significant loss of spectral information compared the 128-channel HERB data over the same energy range, a set of simulations was performed to ascertain the effectiveness of each data type to accurately find the correct parameters of a Band function spectrum. To perform these simulations, a 4-dimensional grid was constructed in parameter space, and each dimension was evenly sampled with 11 input parameter values sufficiently spanning the parameter space. The values were as follows:

- Amplitude: $0.001,0.003,0.01,0.03,0.1,0.3,1.0,3.0,10,31,100$

- $E_{\text {peak }}: 1,3,8,24,69,200,577,1665,4804,13863,40000$

- Low-Energy Index: -2.0, -1.7, -1.4, -1.1, -0.8, -0.5, -0.2, +0.1, +0.4, +0.7, +1.0

- High-Energy Index: -5.0, -4.6, -4.2, -3.8, -3.4, -3.0, -2.6, -2.2, -1.8, -1.4, -1.0

Each grid point comprised a value for each parameter, such that there were $11^{4}$ grid points. At each grid point, a set of 1000 simulated spectra were created with input parameters at that grid point, the resulting photon spectra were folded through the LAD response matrix, and Poisson noise was added to the total and background count rate for each spectrum. The resulting synthetic spectra were then fit with a Band function to determine the spectral parameters. This procedure was completed using the CONT DRM as well as the HERB DRM from Trigger \# 1815. Note that in many cases the combination of parameters of the simulated spectrum may represent an extreme parametrization of the Band function, which may not necessarily reflect the typical observed GRB spectrum. 
The resulting spectral fits to the simulations were compiled, and the mean and standard deviation of each set of 1000 simulations was computed for each grid point. Finally, for each individual input parameter value, the distance between the parameter value of the fit and the input was calculated, integrating over all other dimensions of the sample parameter space. Figure 29 shows the fraction of spectral fits that contain the input spectral parameter within the $1 \sigma, 2 \sigma$, and $3 \sigma$ confidence intervals for each parameter while integrating over the other dimensions of the parameter space. The key result from Figure 29 is that there appears to be minimal loss of spectral information when using the 16-channel CONT data compared to the 128-Channel HERB data.

\section{Appendix B}

\section{Derivation of $E_{\text {peak }}$ for GLOGE}

The GLOGE model is parametrized with $E_{c e n}$, which is the centroid energy in $\mathrm{keV}$, and $s$ which is the standard deviation in units of $\log _{10} \mathrm{keV}$ and is related to the full-width at half-maximum (FWHM) by $s=\mathrm{FWHM} / 2.35482$. Although it is not parametrized with $E_{\text {peak }}$, the power density spectrum is a parabolic function in log-log space, therefore, there will always exist an $E_{\text {peak }}$ although it is not guaranteed to exist within the detector bandpass. It is relatively easy to derive $E_{\text {peak }}$ for GLOGE, which can be found by

$$
\frac{d}{d E}\left(\nu F_{\nu}\right)=0
$$

where $\nu F_{\nu}=E^{2} f_{G L O G E}(E)$. Solving this equation for $E_{\text {peak }}$ results in

$$
E_{\text {peak }}=E_{c e n} e^{2 d s^{2}}
$$

where $d=\ln ^{2}(10)$. In order to correctly calculate the errors on the derived $E_{\text {peak }}$, the errors in $E_{c e n}$ and $s$ must be propagated properly. Assuming higher-order terms in the Taylor 
expansion of the equation are negligible, the error to first order is given by

$$
\sigma_{E_{\text {peak }}}^{2}=\left|\frac{\partial E_{\text {peak }}}{\partial E_{c e n}}\right|^{2} \sigma_{E_{c e n}}^{2}+\left|\frac{\partial E_{\text {peak }}}{\partial s}\right|^{2} \sigma_{s}^{2}+2 \frac{\partial E_{\text {peak }}}{\partial E_{c e n}} \frac{\partial E_{\text {peak }}}{\partial s} \operatorname{Cov}\left(E_{c e n}, s\right)
$$

where $\operatorname{Cov}\left(E_{c e n}, s\right)$ is the covariance between $E_{c e n}$ and $s$. Once the partial derivatives have been determined,

$$
\begin{gathered}
\frac{\partial E_{\text {peak }}}{\partial E_{c e n}}=\frac{E_{\text {peak }}}{E_{c e n}} \\
\frac{\partial E_{\text {peak }}}{\partial \sigma}=4 d s E_{\text {peak }}
\end{gathered}
$$

and we have the covariance between $E_{c e n}$ and $s$ (from the covariance matrix returned after performing the spectral fit) we can properly calculate the error on $E_{\text {peak }}$. 


\section{Appendix C}

\section{$\chi^{2}$ Distributions}

Although the least-squares fitting process did not minimize $\chi^{2}$ as a figure of merit, we can calculate the $\chi^{2}$ goodness-of-fit statistic comparing the model to the data. To do this, we difference the background-subtracted count rates from the model rates, summing first over all energy channels in each detector, and then over all detectors. This is shown by

$$
\chi^{2}=\sum_{i} \sum_{j}\left[\frac{O_{i j}-B_{i j}-M_{i j}}{\sqrt{\sigma_{M_{i j}}^{2}}}\right]^{2},
$$

where the $O_{i j}$ are the observed count rates, $B_{i j}$ are the background rates, $M_{i j}$ are the

model rates, and $\sigma_{M_{i j}}^{2}$ are the derived model variances. In the ideal situation, and assuming acceptable spectral fits (i.e. when performing spectral analysis of simulated data), the reduced $\chi^{2}$ value $\left(\chi^{2} /\right.$ d.o.f. $)$ will tend to distribute around a value of 1 . In this way, $\chi^{2}$ gives an estimate on the acceptability of the fit. Figure 27 shows the reduced $\chi^{2}$ distributions for both the fluence and peak flux spectra, as well as the corresponding BEST distributions. It is important to note that the distributions peak at slightly larger or smaller values than 1, which is acceptable since even the BEST spectral fits represent a rough approximation to the actual spectra of GRBs due to their empirical nature. The fluence $\chi^{2}$ distributions appear similar to the peak flux $\chi^{2}$ distributions even though the fluence spectra contain longer time integration intervals and many GRBs experience spectral evolution. The peak flux sample captures the spectra of all bursts in a small slice of time at the same stage of the lightcurve, meanwhile the fluence sample integrates over the duration of the emission, in many cases over several pulses. The proximity of most of the BEST reduced $\chi^{2}$ values to the nominal value is indicative of acceptable spectral fits.

In addition, Figure 28 plots the BEST reduced $\chi^{2}$ as a function of photon fluence and peak photon flux for the fluence and peak flux spectra, respectively. The reduced $\chi^{2}$ for 
the fluence fits shows a slight upward trend as the photon fluence increases. The average reduced $\chi^{2}$ starts at approximately a value of unity at a low fluence of $\sim 0.2$ photons $\mathrm{cm}^{-2}$ and increases to a value of $\sim 2$ at $\sim 1000$ photons $\mathrm{cm}^{-2}$. Additionally there are several outliers to the trend that exist at high fluence and exhibit even larger reduced $\chi^{2}$ values. This indicates that the goodness- of-fit is increasingly worse the more fluent the burst. This follows from the fact that in most cases extremely fluent bursts are long and may exhibit significant spectral evolution, therefore the time-integrated spectral fit will average over the evolution and will produce a significantly worse fit. When inspecting the reduced $\chi^{2}$ as a function of the photon flux, we find that the trend is flat until $\sim 20$ photons $\mathrm{cm}^{-2}$. Most of the large reduced $\chi^{2}$ values from the peak flux spectra result from higher flux bursts where systematic uncertainties tend to dominate the statistical uncertainties. 


\section{Appendix D}

\section{Simulations of Spectral Parameters from Summing Detectors}

For this catalog, we have chosen to sum appropriate detectors for each burst to perform a spectral analysis. This increases the signal-to-noise ratio of the summed datasets when compared to using single detectors, which increases the amount of signal investigated. This method also provides a simple, objective approach to selecting signal from a GRB on which to do analysis. There are, however, a few consequences to summing detector counts prior to spectral analysis. One of these consequences is that a new DRM for the summed detectors must be created. The DRMs, which contain the effective area of each detector as a function of detector-to-source angle and energy, can be summed to produce a new DRM appropriate for the summed detectors. The photon energy and channel energy edges must be averaged accordingly to provide an accurate mapping from channel to photon energy. Because of this averaging, spectral resolution decreases. As shown in Figure 30, the effective area integrated over photon energies for the summed DRM can average features found in the individual DRMs. For example, the typical peak response energy for BATSE detector is 60-70 keV, and while the summed DRM preserves this approximately, the region around the peak response energy is slightly broadened due to the summation of the DRMs.

In addition to the loss of spectral resolution, there may be additional systematic errors associated with performing summed spectral fits. To investigate these effects, we have performed several Monte Carlo simulations of GRB spectra and compared the results from summing detectors to the joint spectral analysis. For each simulation, we chose a single GRB that was best fit by each model used in this catalog. We created 20,000 simulated spectra for each burst using the best fit fluence spectral parameters. Half of the spectra for each burst were created using the summed detector and response, and the other half were created using the individual detectors and responses. Each simulated 
spectrum was produced with a Poisson deviate background and convolved through the detectors' response to produce an accurate representation of an actual GRB observed by that detector. Each background-subtracted simulated spectrum was then folded through the associated DRM(s) and fit with the corresponding best fit function. The parameter distributions resulting from the simulations are shown in Figures 31, 34 . It is evident that the parameter estimates between the two methods are similar. No single parameter has a mean value that deviates more than $2 \%$ when comparing the summed and joint detectors, and in general the deviations are much less. In all cases the $1 \sigma$ standard deviations of the summed spectral parameters are slightly larger than those of the joint spectral parameters. This increased variance is the systematic error that is added to the parameter estimations when the detectors are summed. In all cases the difference in the standard deviation is a small fraction of one standard deviation.

Another potential consequence of summing detectors is that the resulting fit statistics could be skewed. To measure the amount by which the $\chi^{2}$ distributions are affected, we compare the $\chi^{2}$ distributions of the simulated joint fits and the simulated summed fits. Since a distribution of $\chi^{2}$ values from fitting a model to the data should be a $\chi^{2}$ distribution, we expect that when a BAND function is fit to the simulated data produced from a BAND function, the resulting distribution of $\chi^{2}$ should also be a $\chi^{2}$ distribution defined by the number of degrees of freedom of the fit. However, the figure of merit we are minimizing is not $\chi^{2}$, but $\mathrm{C}$-stat, therefore the resulting distribution is not necessarily expected to represent the ideal case. In Figure 35 we show that indeed both the joint and summed $\chi^{2}$ distributions deviate from the distribution that is expected in the ideal case. By fitting a $\chi^{2}$ distribution to the distribution of $\chi^{2}$, we can estimate the amount of deviation between what is expected and what is produced through the simulations. In this case, we find that both the joint and summed $\chi^{2}$ distributions deviate by the same amount, therefore we conclude that this shift is due to the minimization of C-stat (instead of $\chi^{2}$ ) and not 
caused by summing the detectors. Additionally, we can inspect the reduced $\chi^{2}$, which can be used as a measure of the goodness-of-fit and also represents a comparison between the data and model variances. Ideally, a reduced $\chi^{2}$ distribution will peak close to a value of unity, denoting that the model and data variances are as expected. A value less than 1 implies that the model variances are overestimated, and a value greater than 1 implies that the model variances are underestimated. As shown in Figure 36, the joint and summed reduced $\chi^{2}$ distributions in many cases are not the same. In most cases, the medians of the distributions are the same, but the summed distribution is much broader. This indicates that summing the detectors tends to change the model variance.

For the purpose of model comparison, $\chi^{2}$ is an important statistic, and we need to determine if $\Delta \chi^{2}$ changes when summing detectors. For each of the bursts on which we performed simulations, we also fit the other models to determine how accurate the summed detectors method is at model selection. For this test, we calculate the change in $\chi^{2}$ and plot it as a distribution for each simulation. We then determine the change in the degrees of freedom when fitting each model and the cutoff value we have used for this catalog. Because of the additive property of $\chi^{2}$, the $\Delta \chi^{2}$ values are associated with a $\chi^{2}$ distribution represented by the difference in the degrees of freedom between the two models, therefore our criteria of 6 units of $\chi^{2}$ per difference in degrees of freedom represents a cutoff in a $\chi^{2}$ distribution. This cutoff value admits a probability in achieving our predetermined change in $\chi^{2}$ by chance, so for the summed $\Delta \chi^{2}$ distributions to be acceptable, the percentage of the distribution existing above the cutoff must be approximately the same as that from the joint distribution. This would indicate that both methods produce the same model choice at the same probability. Figure 37 shows an example of the comparison of $\Delta \chi^{2}$ between joint and summed fits. Even though the distributions of $\Delta \chi^{2}$ are different in many cases, approximately the same amount of the summed distribution exists above the cutoff when compared to the joint distribution. 
The culmination of the simulation tests between joint fitting of detectors and summing detectors indicates a relatively small loss of information by adding detector count rates and responses prior to fitting. The details in the response are lost through the summation process, although since the 16-channel CONT data does not provide high spectral resolution, this effect is minimal. The parameter values from the fits vary only negligibly, and the parameter errors are only slightly larger when summing the detectors. The $\chi^{2}$ values that are produced from summed fits slightly deviate from a $\chi^{2}$ distribution of the required number of degrees of freedom, but they deviate no more than the joint fit $\chi^{2}$, therefore they are unaffected by the summation. The reduced $\chi^{2}$, which measures the goodness-of-fit, can however, show significant deviation. The means of the reduced $\chi^{2}$ distributions are typically similar, although their distance from unity can be larger than expected. In particular, if a fit is required to be within $1 \sigma$ of the mean $(\sim 1)$, the result from the summed detectors will return a small percentage of fits that would have been rejected if fitted with the joint detectors. Finally, the $\Delta \chi^{2}$ found when comparing models using the summed detectors will succeed in choosing the same model almost all of the time when compared to fitting joint detectors. From these results, we show that summing detectors for this catalog has little adverse effect on the spectral results. 


\section{Appendix E Spectral Table Formats}

Please note that the spectral tables list the raw results of each spectral fit to each GRB. In cases where the spectral fit failed, the values reported are those that initialized the spectral fit. If the uncertainty on the spectral parameters is reported as zero (no uncertainty), then the fit failed. In a few cases throughout these tables, the uncertainties for certain spectral parameters may be reported as '9999.99' which indicates that the uncertainty on that parameter is completely unconstrained. An example of this is when the spectral data from a burst is fitted with a BAND function but is unable to constrain the high-energy index. In this case, the best fit centroid value of the high-energy index parameter is reported, and the '9999.99' is reported for the uncertainty. 
Table 1. BAND Fluence \& Peak Flux Parameters Table Format

\begin{tabular}{|c|c|c|}
\hline Column & Format & Description \\
\hline 1 & $\mathrm{I} 4$ & Trigger number \\
\hline 2 & A4 & Detectors used \\
\hline 3 & A4 & Datatype \\
\hline 4 & F6.2 & Total integrated time \\
\hline 5 & $\mathrm{E} 8.2$ & Amplitude \\
\hline 6 & $\mathrm{E} 8.2$ & Uncertainty in Amplitude \\
\hline 7 & F7.2 & Low-Energy Spectral Index \\
\hline 8 & F7.2 & Uncertainty in Low-Energy Spectral Index \\
\hline 9 & F 7.2 & High-Energy Spectral Index \\
\hline 10 & F 7.2 & Uncertainty in High-Energy Spectral Index \\
\hline 11 & $\mathrm{E} 8.2$ & $E_{\text {peak }}$ \\
\hline 12 & $\mathrm{E} 8.2$ & Uncertainty in $E_{\text {peak }}$ \\
\hline 13 & F7.2 & Photon Flux \\
\hline 14 & F7.2 & Uncertainty in Photon Flux \\
\hline 15 & F7.2 & Photon Fluence \\
\hline 16 & F7.2 & Uncertainty in Photon Fluence \\
\hline 17 & $\mathrm{E} 9.2$ & Energy Flux \\
\hline 18 & E9.2 & Uncertainty in Energy Flux \\
\hline 19 & E9.2 & Energy Fluence \\
\hline 20 & E9.2 & Uncertainty in Energy Fluence \\
\hline 21 & F8.2 & $\chi^{2}$ Goodness-of-Fit Statistic \\
\hline 22 & $\mathrm{I} 2$ & Degrees of Freedom \\
\hline
\end{tabular}


Table 2. COMP Fluence \& Peak Flux Parameters Table Format

\begin{tabular}{lll}
\hline Column & Format & Description \\
\hline 1 & I4 & Trigger number \\
2 & A4 & Detectors used \\
3 & A4 & Datatype \\
4 & F6.2 & Total integrated time \\
5 & E8.2 & Amplitude \\
6 & E8.2 & Uncertainty in Amplitude \\
7 & F7.2 & Spectral Index \\
8 & F7.2 & Uncertainty in Spectral Index \\
9 & E8.2 & E peak \\
10 & E8.2 & Uncertainty in $E_{\text {peak }}$ \\
11 & F7.2 & Photon Flux \\
12 & F7.2 & Uncertainty in Photon Flux \\
13 & F7.2 & Photon Fluence \\
14 & F7.2 & Uncertainty in Photon Fluence \\
15 & E9.2 & Energy Flux \\
16 & E9.2 & Uncertainty in Energy Flux \\
17 & E9.2 & Energy Fluence \\
18 & E9.2 & Uncertainty in Energy Fluence \\
19 & F8.2 & $\chi^{2}$ Goodness-of-Fit Statistic \\
20 & I2 & Degrees of Freedom \\
\hline & & \\
\hline 1 & &
\end{tabular}


Table 3. GLOGE Fluence \& Peak Flux Parameters Table Format

\begin{tabular}{lll}
\hline Column & Format & Description \\
\hline 1 & I4 & Trigger number \\
2 & A4 & Detectors used \\
3 & A4 & Datatype \\
4 & F6.2 & Total integrated time \\
5 & E8.2 & Amplitude \\
6 & E8.2 & Uncertainty in Amplitude \\
7 & F6.2 & Full-Width at Half Maximum \\
8 & F6.2 & Uncertainty in Full-Width Half Maximum \\
9 & E8.2 & Centroid Energy \\
10 & E8.2 & Uncertainty in Centroid Energy \\
11 & E8.2 & E peak \\
12 & E8.2 & Uncertainty in E peak \\
13 & F7.2 & Photon Flux \\
14 & F7.2 & Uncertainty in Photon Flux \\
15 & F7.2 & Photon Fluence \\
16 & F7.2 & Uncertainty in Photon Fluence \\
17 & E9.2 & Energy Flux \\
18 & E9.2 & Uncertainty in Energy Flux \\
19 & E9.2 & Energy Fluence \\
20 & E9.2 & Uncertainty in Energy Fluence \\
21 & F8.2 & $\chi^{2}$ Goodness-of-Fit Statistic \\
\hline & I2 & Degrees of Freedom \\
\hline 2 & & \\
\hline & & \\
14 & &
\end{tabular}


Table 4. PL Fluence \& Peak Flux Parameters Table Format

\begin{tabular}{lll}
\hline Column & Format & Description \\
\hline 1 & I4 & Trigger number \\
2 & A4 & Detectors used \\
3 & A4 & Datatype \\
4 & F6.2 & Total integrated time \\
5 & E8.2 & Amplitude \\
6 & E8.2 & Uncertainty in Amplitude \\
7 & F7.2 & Spectral Index \\
8 & F7.2 & Uncertainty in Spectral Index \\
9 & F7.2 & Photon Flux \\
10 & F7.2 & Uncertainty in Photon Flux \\
11 & F7.2 & Photon Fluence \\
12 & F7.2 & Uncertainty in Photon Fluence \\
13 & E9.2 & Energy Flux \\
14 & E9.2 & Uncertainty in Energy Flux \\
15 & E9.2 & Energy Fluence \\
16 & E9.2 & Uncertainty in Energy Fluence \\
17 & F8.2 & $\chi^{2}$ Goodness-of-Fit Statistic \\
18 & I2 & Degrees of Freedom \\
\hline
\end{tabular}


Table 5. SBPL Fluence \& Peak Flux Parameters Table Format

\begin{tabular}{|c|c|c|}
\hline Column & Format & Description \\
\hline 1 & I4 & Trigger number \\
\hline 2 & A4 & Detectors used \\
\hline 3 & A4 & Datatype \\
\hline 4 & F6.2 & Total integrated time \\
\hline 5 & $\mathrm{E} 8.2$ & Amplitude \\
\hline 6 & $\mathrm{E} 8.2$ & Uncertainty in Amplitude \\
\hline 7 & F 7.2 & Low-Energy Spectral Index \\
\hline 8 & F7.2 & Uncertainty in Low-Energy Spectral Index \\
\hline 9 & F7.2 & High-Energy Spectral Index \\
\hline 10 & F7.2 & Uncertainty in High-Energy Spectral Index \\
\hline 11 & $\mathrm{E} 8.2$ & Spectral Break Energy \\
\hline 12 & $\mathrm{E} 8.2$ & Uncertainty in Spectral Break Energy \\
\hline 13 & $\mathrm{E} 8.2$ & $E_{\text {peak }}$ \\
\hline 14 & $\mathrm{E} 8.2$ & Uncertainty in $E_{\text {peak }}$ \\
\hline 15 & F7.2 & Photon Flux \\
\hline 16 & F7.2 & Uncertainty in Photon Flux \\
\hline 17 & F7.2 & Photon Fluence \\
\hline 18 & F7.2 & Uncertainty in Photon Fluence \\
\hline 19 & E9.2 & Energy Flux \\
\hline 20 & E9.2 & Uncertainty in Energy Flux \\
\hline 21 & E9.2 & Energy Fluence \\
\hline 22 & E9.2 & Uncertainty in Energy Fluence \\
\hline 23 & F8.2 & $\chi^{2}$ Goodness-of-Fit Statistic \\
\hline 24 & $\mathrm{I} 2$ & Degrees of Freedom \\
\hline
\end{tabular}




\section{REFERENCES}

Atteia, J. L. \& Boer, M. 2011, Comptes Rendus Physique, 12, 255

Band D. L., Ford L., Matteson, J., et al. 1992, Experimental Astronomy, 2, 307

Band, D. L., et al. 1993, ApJ, 413, 281

Band, D. L. \& Preece, R. D. 2005, ApJ, 627, 319

Barthelmy, S. D., et al. 1994, AIP Conf. Proc., 307, Gamma-ray bursts: Second workshop, ed. G. J. Fishman, J. J. Brainerd, \& K. Hurley, 643

Bhat, P. N., Fishman, G. J., Meegan, C. A., Wilson, R. B., Brock, M. N., \& Paciesas, W. S. 1992, Nature, 359, 217

Briggs, M. S., et al. 1994, ApJ, 431, 416

Briggs, M. S., et al. 1996, AIP Conf. Proc. 385, Gamma-ray Bursts: 3rd Huntsville Symposium, ed. C. Kouveliotou, M. S. Briggs, G. J. Fishman, 335

Briggs, M. S., Band, D. L., Kippen, R. M., et al. 1999, ApJ, 524, 82

Bromberg, O., Nakar, E., Piran, T., \& Sari, R. 2012, ApJ, 749, 110

Cash, W. 1979, ApJ, 228, 939

Cline, T. L., Desai, U. D., Klebesadel, R. W., \& Strong, I. B. 1973, ApJ, 185, L1

De Pasquale, M., Piro, L., Gendre, B., et al. 2006, A\&A, 455, 813

Dermer, C. ApJ, 501, L157

Dezalay, J. P., Barat, C., Talon, R., Sunyaev, R., Terekhov, O., Kuznetsov, A. 1992, AIP Conf. Proc., 265, Gamma-ray bursts, ed. W. Paciesas, \& G. J. Fishman, 304 
Dingus, B. L., et al. 1994, AIP Conf. Proc. 307, Gamma-Ray Bursts: Proceedings of the 2nd Workshop, ed. G. J. Fishman, 22

Frontera, F., Guidorzi, C, Montanari, E., et al. 2009, ApJS, 180, 192

Goldstein, A., Preece, R. D., \& Briggs, M. S. 2010, ApJ, 721, 1329

Goldstein, A., et al. 2012, ApJS, 199, 19

Horack, J. M., 1991, Development of the Burst and Transient Source Experiment (BATSE), Washington, DC: NASA

Hrabovsky, G. E., Ogelman, H., Kouveliotou, C., van Paradijs, J., 1996, BAAS, 28, 1198

Kaneko, Y. 2005, Ph.D. dissertation, Univ. Alabama in Huntsville

Kaneko, Y., Preece, R. D., Briggs, M. S., Paciesas, W. S., Meegan, C. A., \& Band, D. L. 2006, ApJS, 166, 298

Klebesadel, R. W., Strong, I. B., \& Olson, R. A. 1973, ApJ, 182, L85

Kouveliotou, C., Meegan, C. A., Fishman, G. J., Bhat, N. P., Briggs, M. S., Koshut, T. M., Paciesas, W. S., \& Pendleton, G. N. 1993, ApJ, 413, L101

Laird, C. E., Harmon, B. A., Wilson C. A., Hunter, D., \& Isaacs, J. 2006, Nuclear Instruments and Methods, 566, 433

Lloyd, N. M., Petrosian, V., \& Mallozzi, R. S. 2000, ApJ, 534, 227

Lloyd-Ronning, N. M. \& Ramirez-Ruiz, E. 2002, ApJ, 576, 101

Mallozzi, R. S., Paciesas, W. S., Pendleton, G. N., Briggs, M. S., Preece, R. D., Meegan, C. A., \& Fishman, G. J. 1995, ApJ, 454, 597

Massaro, F., Grindlay, J. E., \& Paggi, A. 2010, ApJ, 714, L299 
Matz, S. M., Forrest, D. J., Vestrand, W. T., Chupp, E. L., Share, G. H., Rieger, E. 1985, ApJ, 288, L37

Mazets, E. P., et al. 1981, Ap\&SS, 80, 3

McNamara, B. J., Harmon, B., \& Harrison, T. E. 1995, A\&A, 111, 587

Meegan, C. A., Fishman, G. J., Wilson, R. B., et al. 1992, Nature, 355, 14

Mitrofanov, I. G., et al. 1999, ApJ, 522, 1069

Norris, J. P., et al. 1986, Advances in Space Research 6, 26th COSPAR metting, ed. J. Lastovicka, 19

Pelangeon A., Atteia, J. L., Nakagawa, Y. E., et al. 2008, A\&A, 491, 157

Pendleton, G. N., Paciesas, W. S., Briggs, M. S., et al. 1994, AIP Conf. Proc., 304, 749

Pendleton, G. N., et al. 1995, Nuclear Instruments and Methods, 364, 567

Preece, R. D., et al. 1998, ApJ, 496, 849

Preece, R. D., Briggs, M. S., Giblin, T. W., Mallozzi, R. S., Pendleton, G. F., \& Paciesas, W. S. 2002, ApJ, 581, 1248

Roming, P. W. A., Koch, T. S., Oates, S. R., et al. 2009, ApJ, 690, 163

Ryde, F., 1999, Astro. Lett. and Comm., 39, 281

Sakamoto, T., Baumgartner W. H., Cummings, J. R., et al. 2011, ApJS, 195, 2

Sari, R., Narayan, R. \& Piran T. 1996, ApJ, 473, 204

Sari, R. \& Piran T. 1997, ApJ, 485, 270 


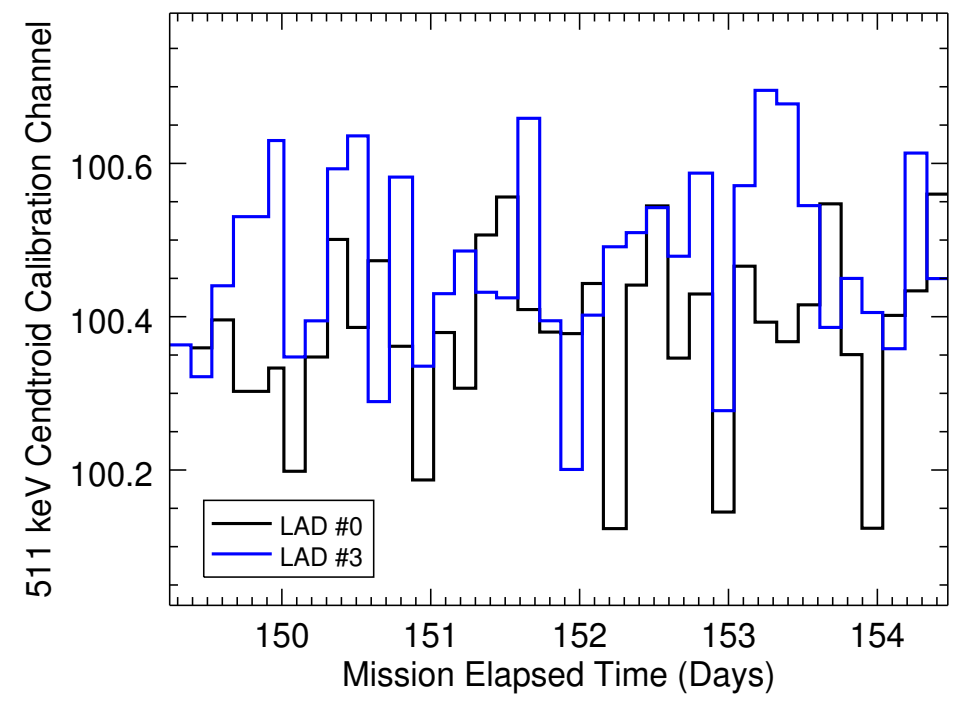

(a) Near Start of Mission

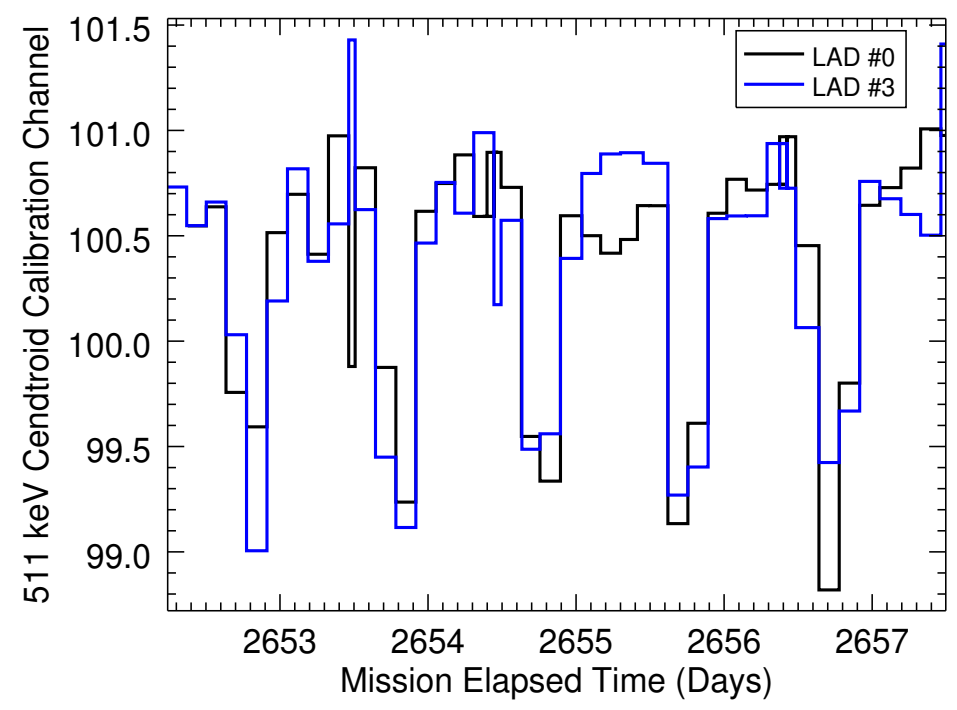

(b) Near End of Mission

Fig. 1.- The variation of the centroid of the $511 \mathrm{keV}$ calibration line in channel space for two detectors from a period of time near the start and end of the mission. 


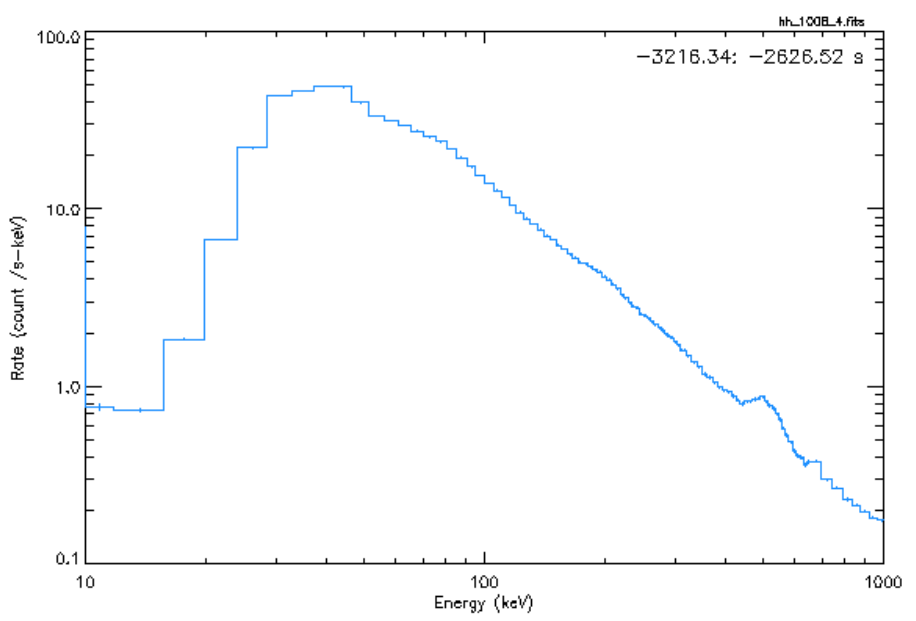

(a) Near Start of Mission

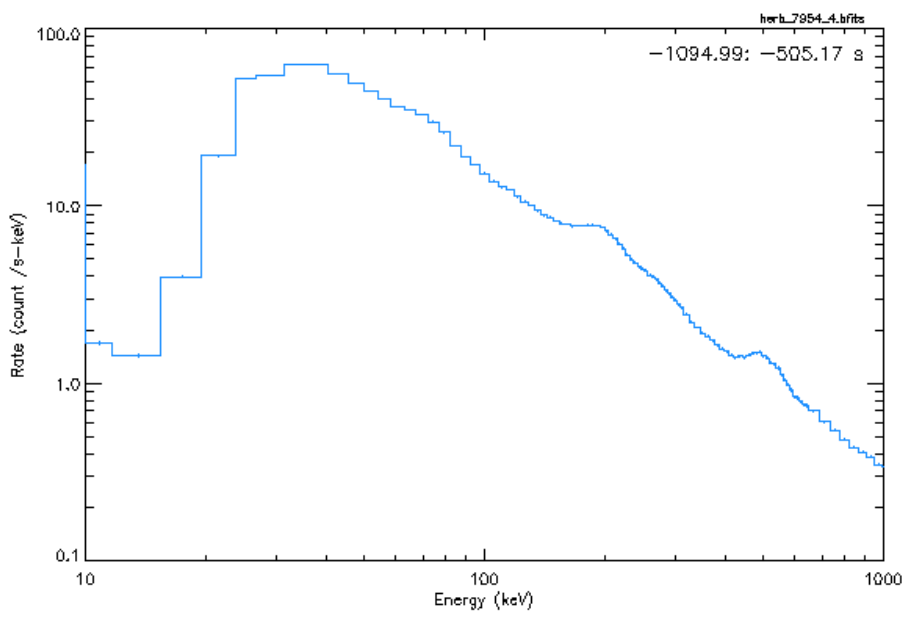

(b) Near End of Mission

Fig. 2. - Spectra of the background from LAD \#4 from near the beginning and end of the BATSE mission. 


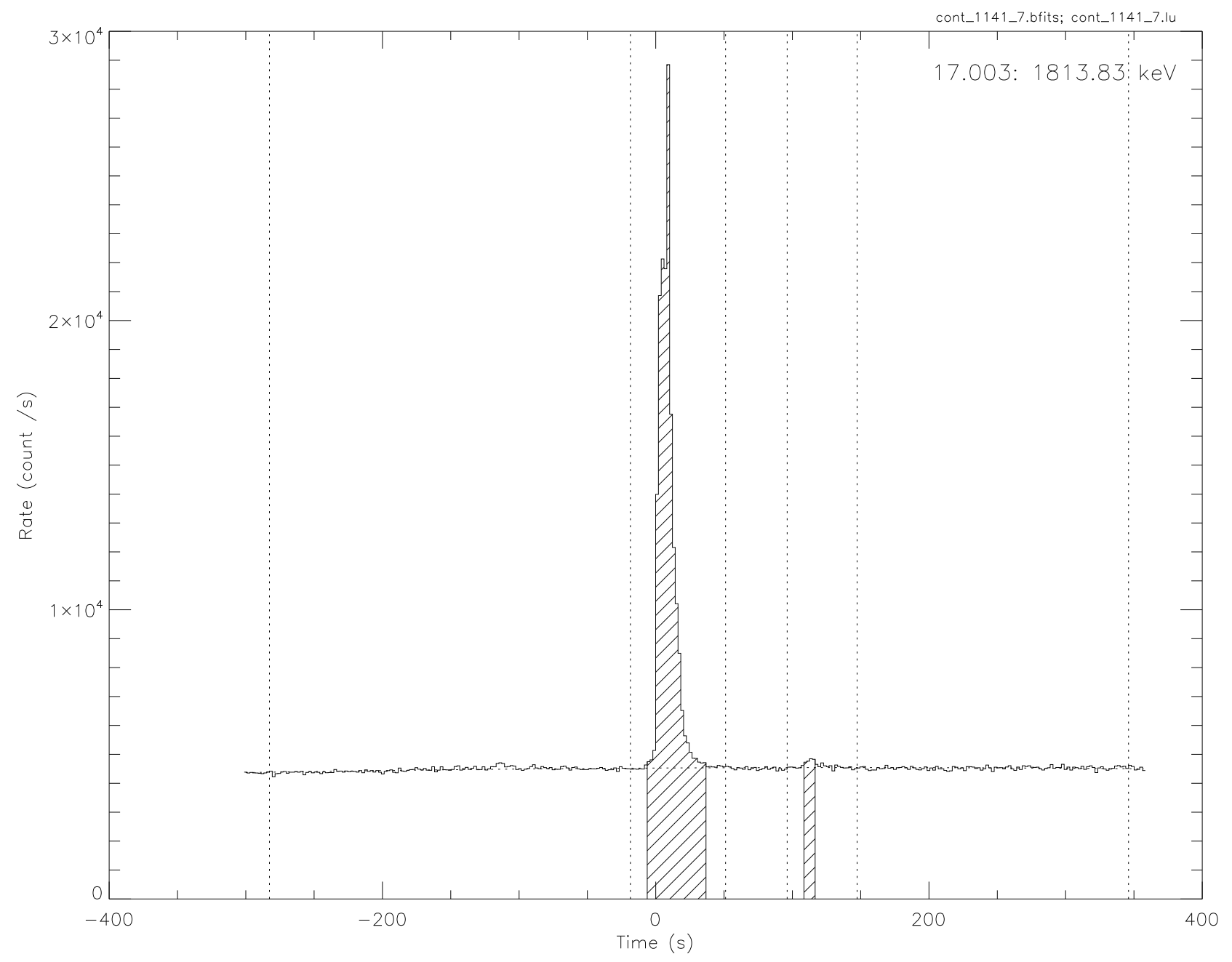

Fig. 3.- An example of the background selections from trigger \#1141. The vertical dotted lines demarcate the three regions in which the background is fit. In this particular example, a second order polynomial was fit to the background regions. 


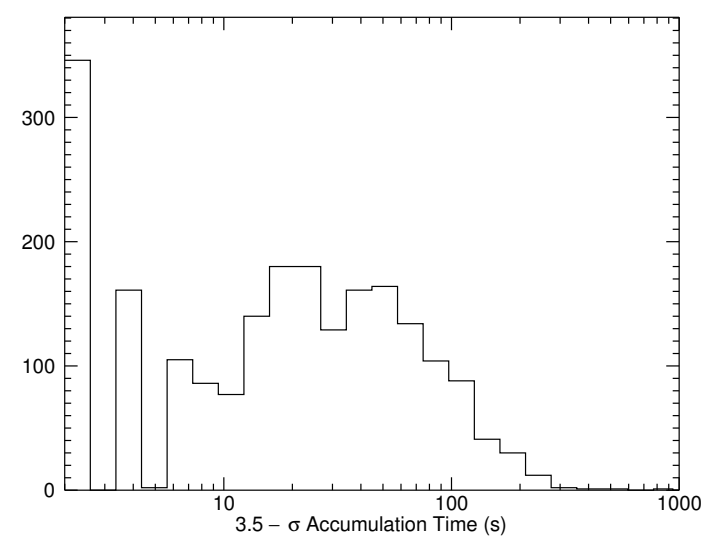

(a)

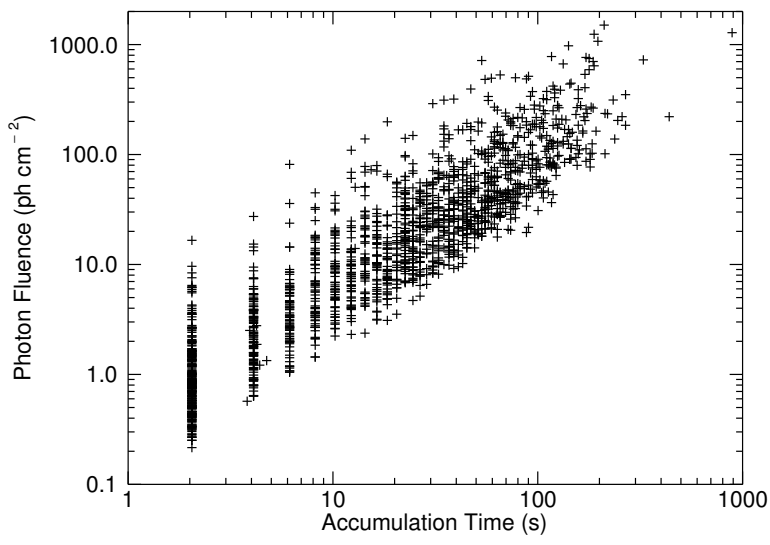

(b)

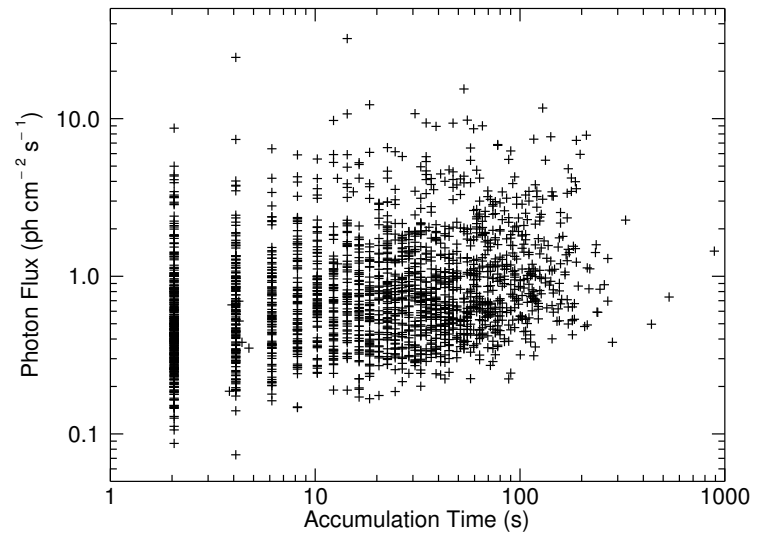

(c)

Fig. 4.- 4(a) is the distribution of the accumulation time based on the $3.5 \sigma$ signal-tonoise selections. Note the similarity to the traditional $T_{90}$ distribution, with the minimum near 1 second. No other estimation of the duration was factored into the production of the accumulation time. 4(b) and 4(c) show the comparison of the model photon fluence and photon flux to the accumulation time respectively. The fluxes and fluences shown in these figures are from the estimated BEST model fits. 


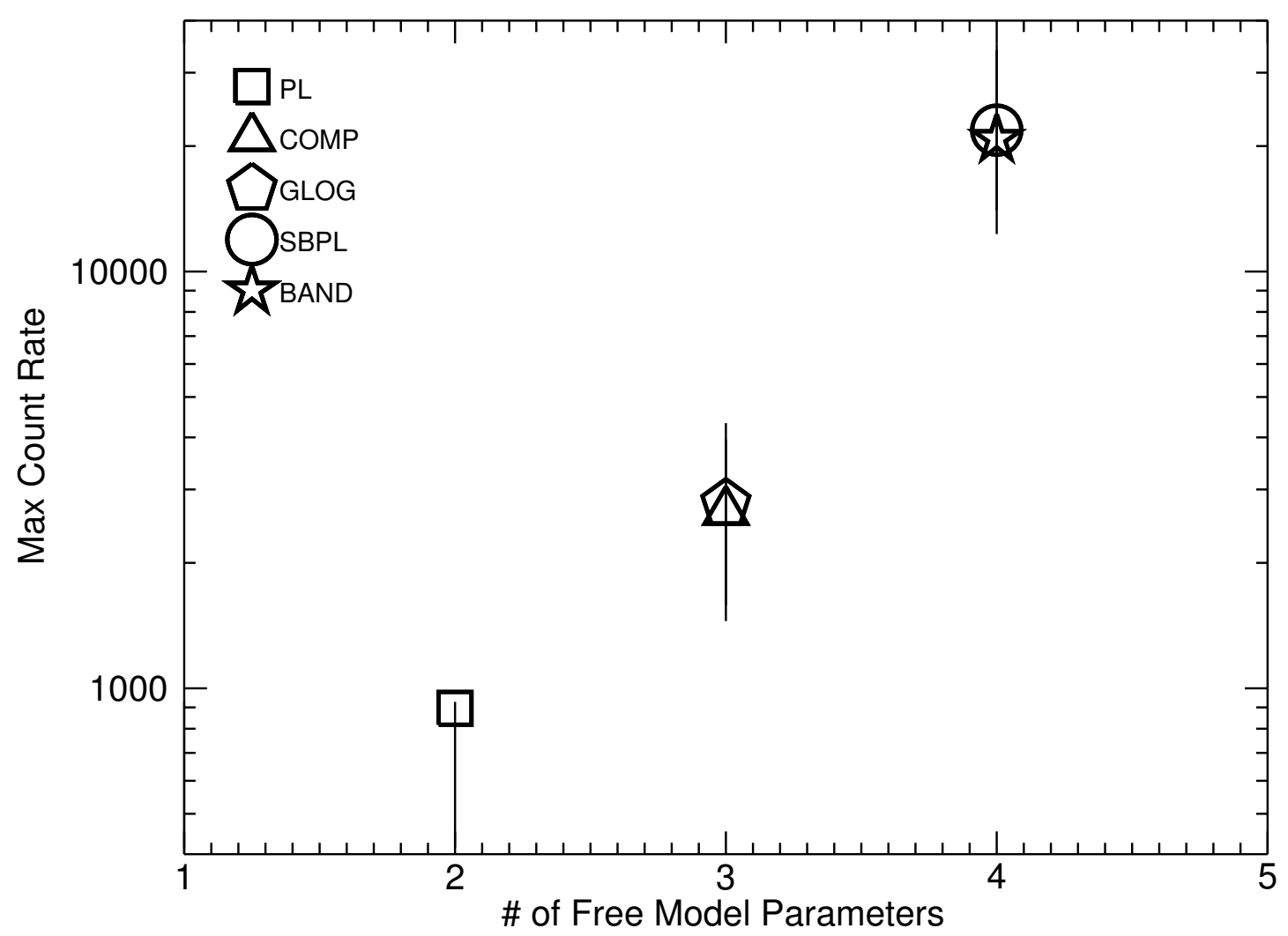

Fig. 5.- Plot of the average maximum background-subtracted count rates (as a proxy for observed intensity) versus the number of degrees of freedom of the best fit model. The maximum count rate is defined as the single $2048 \mathrm{~ms}$ bin that contains the highest backgroundsubtracted count rate. The best fit model was determined for each burst, and a geometric average was calculated for the maximum count rates of the bursts for each best fit model. The error bars shown are the $1 \sigma$ standard deviations of the distributions of maximum count rates for each best fit model. 


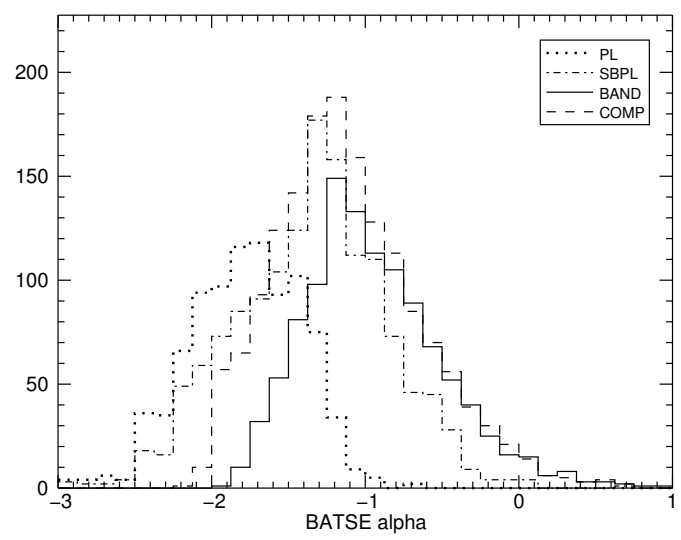

(a)

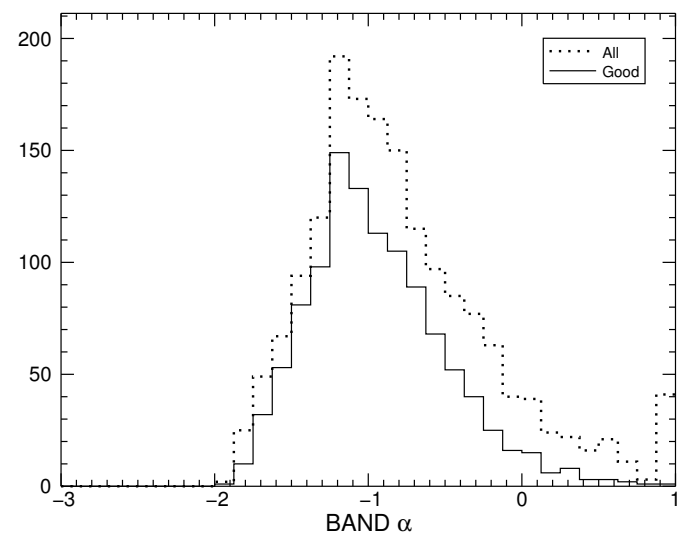

(c)

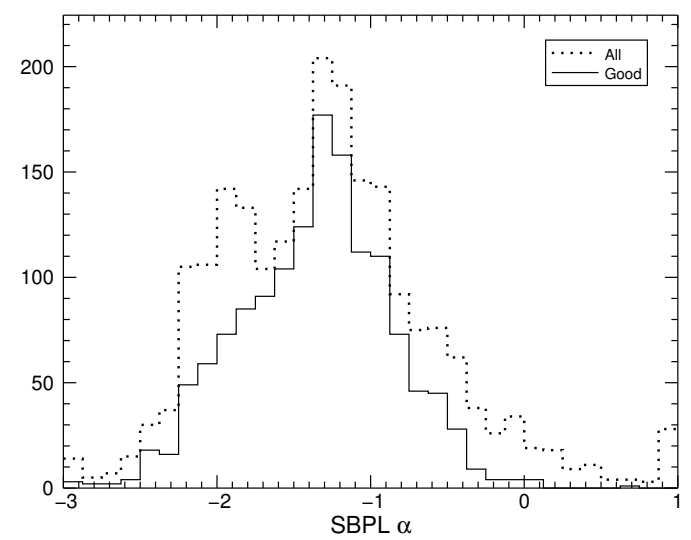

(b)

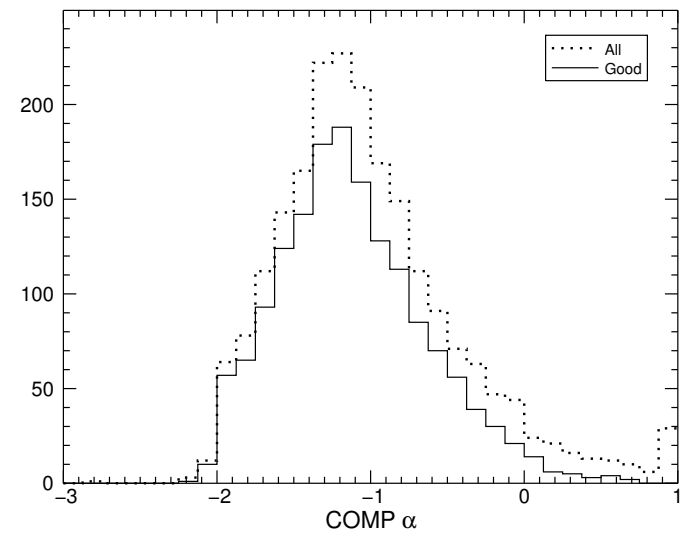

(d)

Fig. 6.- Distributions of the low-energy spectral indices from fluence spectral fits. 6(a) shows the distributions of GOOD parameters and compares to the distribution of PL indices. 6(b) 6(d) display the comparison between the distribution of GOOD parameters and all parameters with no data cuts. The last bin includes values greater than 1 . 


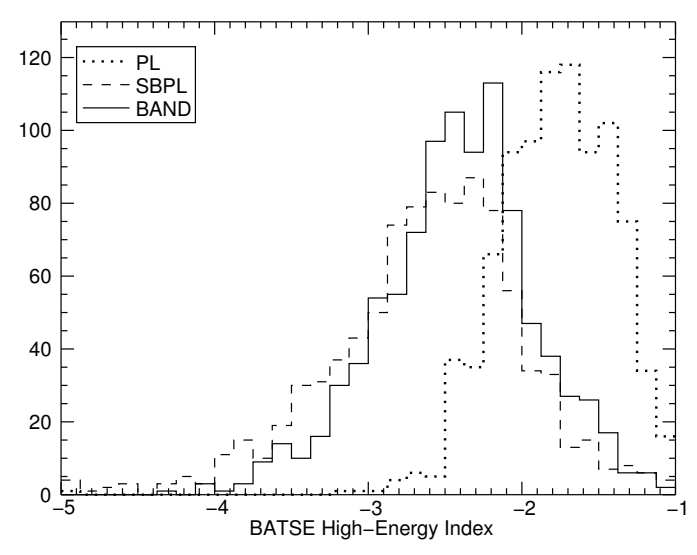

(a)

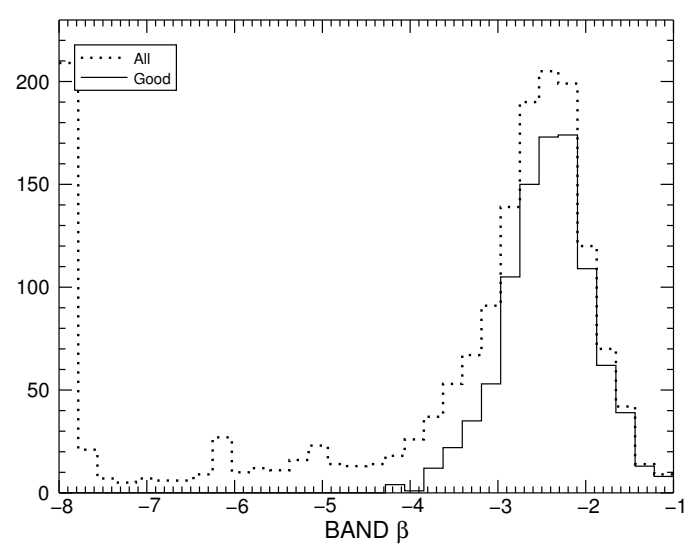

(c)

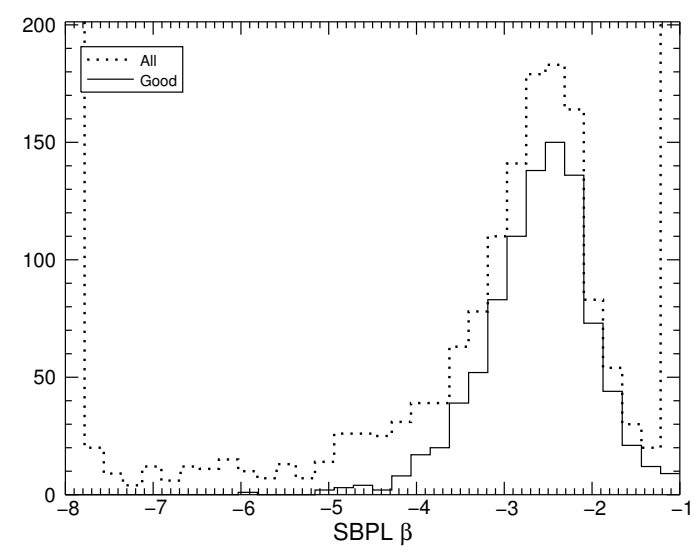

(b)

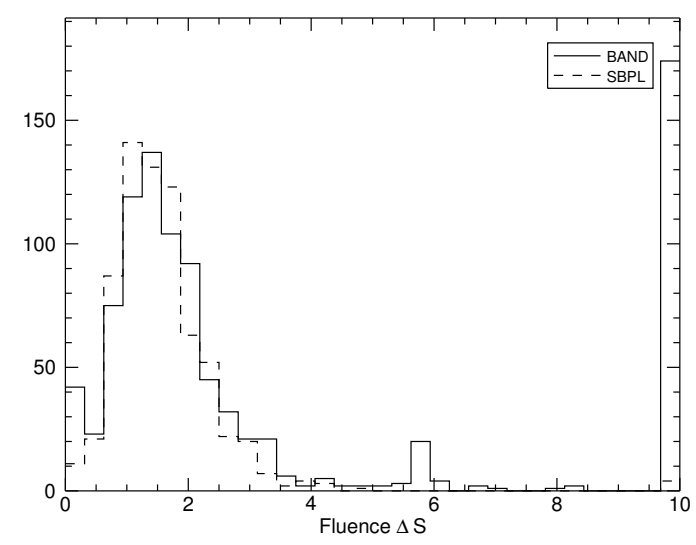

(d)

Fig. 7.- 7(a)-7(c) are distributions of the high-energy spectral indices from fluence spectral fits. $7(\mathrm{a})$ shows the distributions of GOOD parameters and compares to the distribution of PL indices. 7(b) and 7(c) display the comparison between the distribution of GOOD parameters and all parameters with no data cuts. The first bins include values less than -8 and the last bin include values greater than -1. 7(d)] shows the distribution of the difference between the low- and high-energy indices. The first bin contains values less than 0 , indicating that the centroid value of alpha is steeper than the centroid value of beta. 


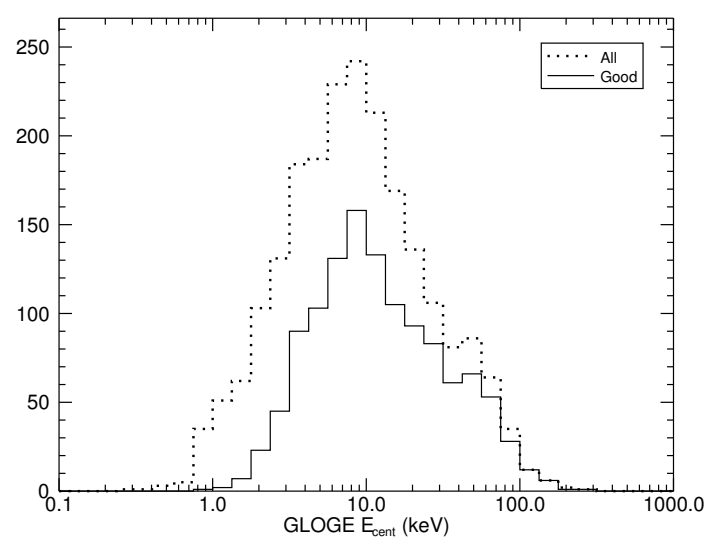

(a)

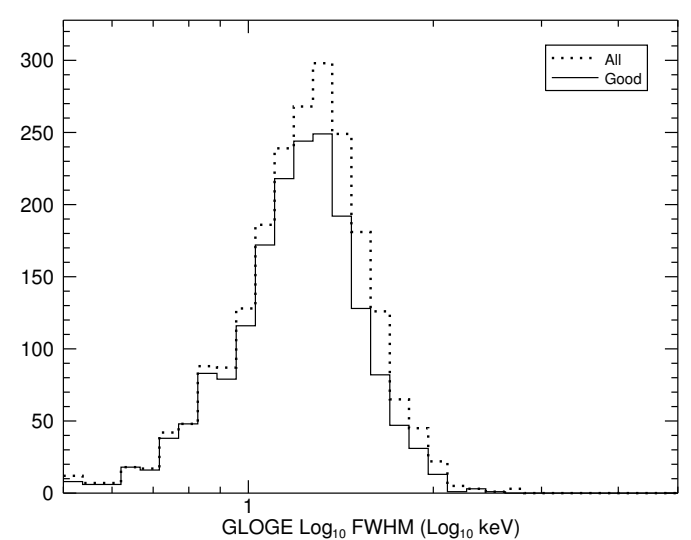

(b)

Fig. 8.- Distributions of the GLOGE $E_{\text {cent }}$ and FWHM parameters from the fluence spectral fits. 


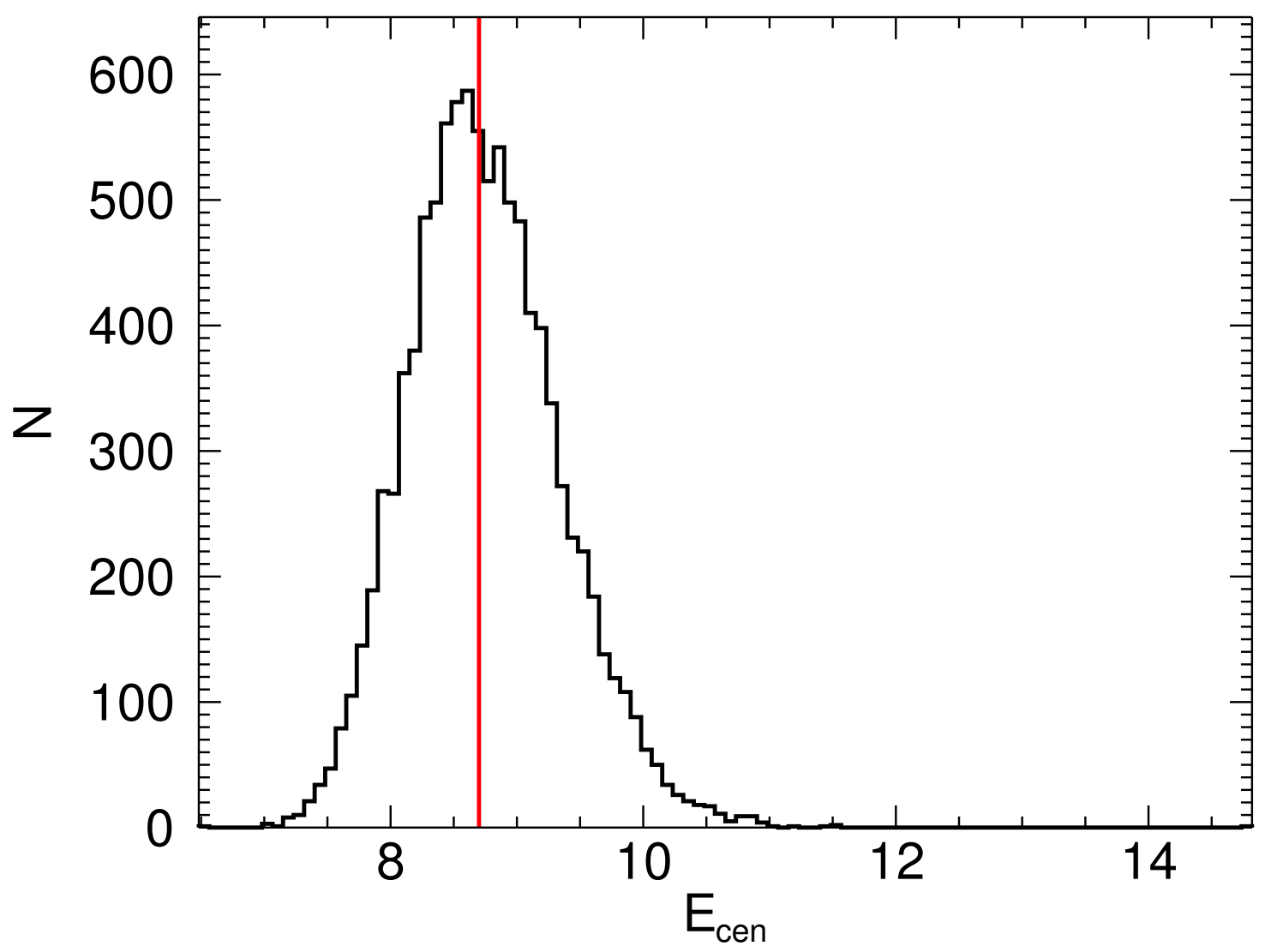

Fig. 9.- Distribution of GLOGE $E_{c e n}$ from fitting synthetic spectra with input $E_{c e n}=8.7$ $\mathrm{keV}$. The red vertical line indicates the input $E_{c e n}$ value. 


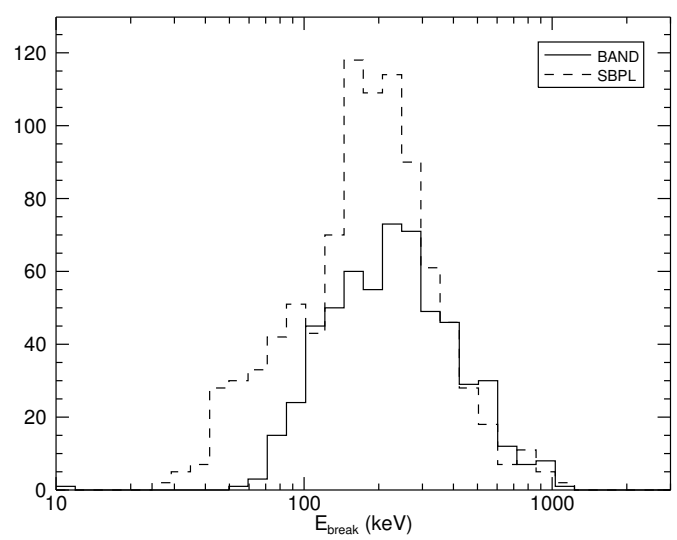

(a)

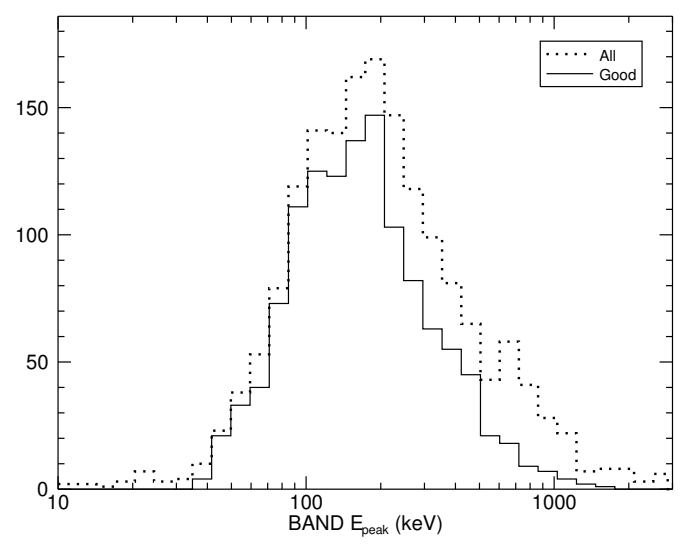

(c)

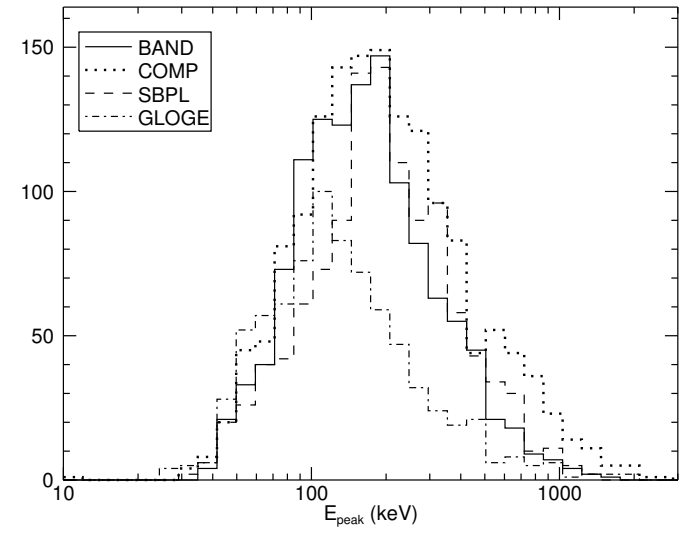

(b)

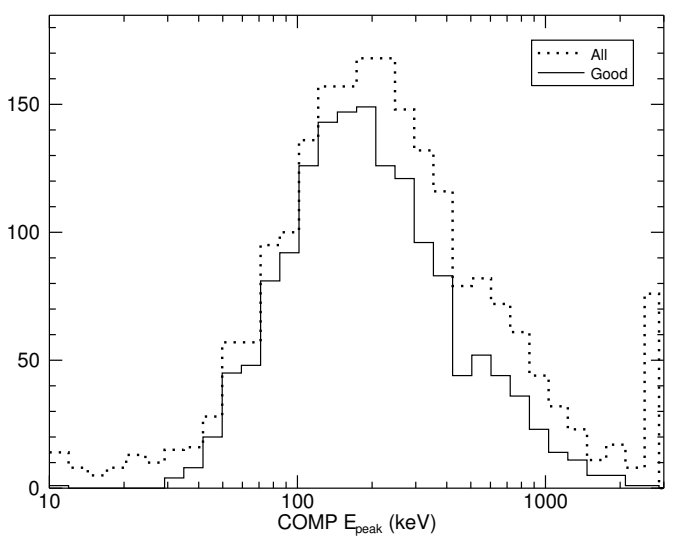

(d)

Fig. 10.- Distributions of $E_{\text {break }}$ and $E_{\text {peak }}$ from fluence spectral fits. 10(a) displays the comparison between the distribution of GOOD $E_{\text {break }}$ and $E_{\text {break }}$ with no data cuts. 10(b) shows the distributions of GOOD $E_{\text {peak }}$ for BAND, SBPL, COMP, and GLOGE. 10(c) and 10(d) display the comparison between the distribution of GOOD paramters and all parameters with no data cuts. 


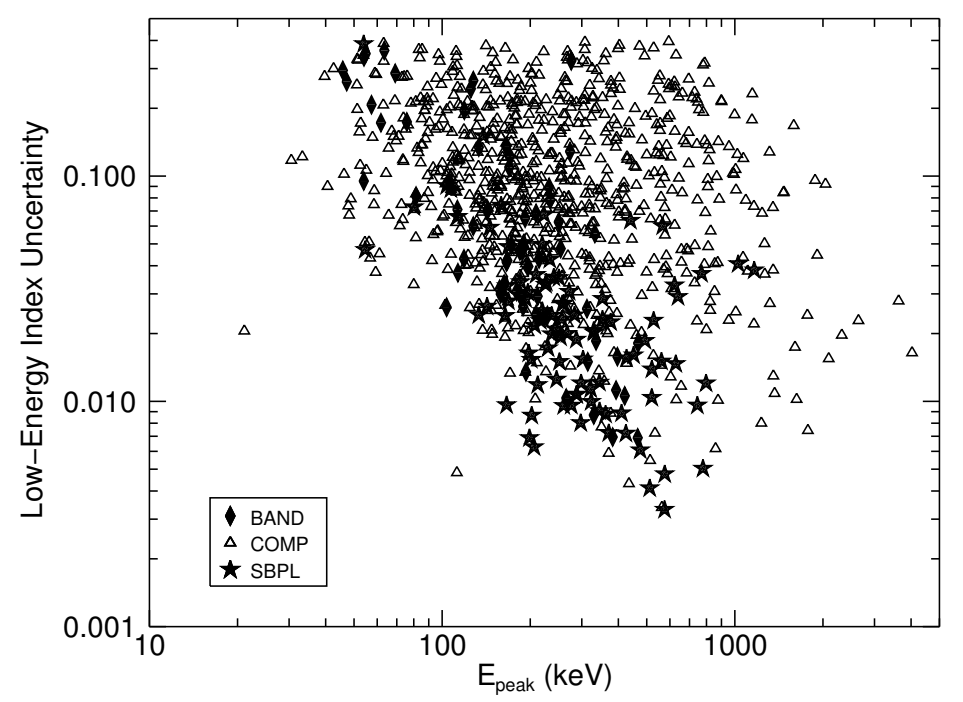

Fig. 11. - Comparison of the low-energy index uncertainty and $E_{\text {peak }}$ for three models from the fluence spectral fits. This comparison reveals a correlation between the $E_{\text {peak }}$ energy and the uncertainty on the low-energy index: generally a lower energy $E_{\text {peak }}$ tends to result in a less constrained low-energy index. 


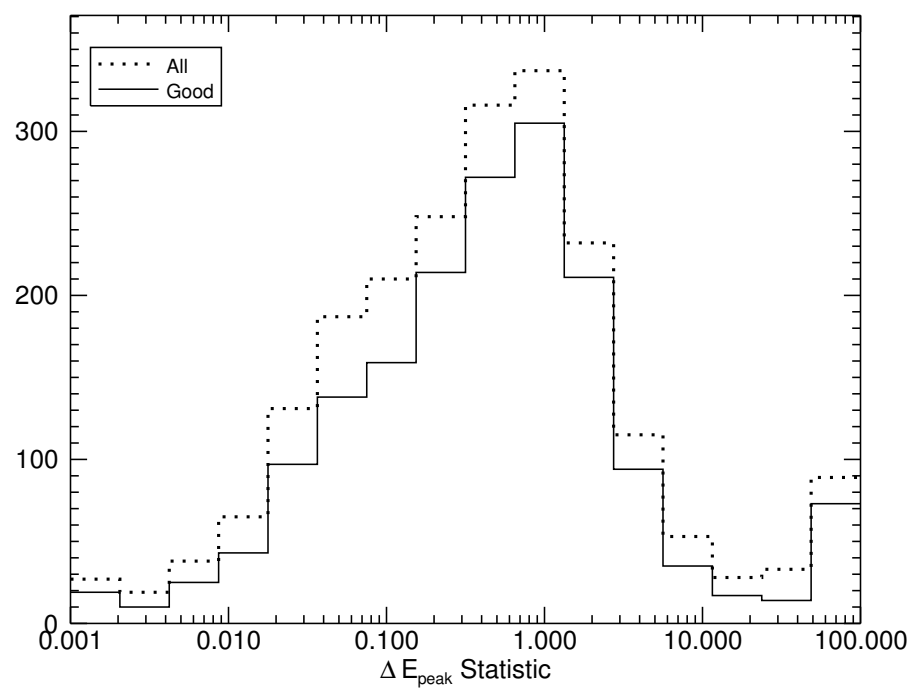

Fig. 12.- Distribution of the $\Delta E_{\text {peak }}$ statistic for the COMP and BAND models from fluence spectral fits. A value less than 1 indicates the $E_{\text {peak }}$ values are similar within errors, while a value larger than 1 indicates the $E_{\text {peak }}$ values are not within errors. 


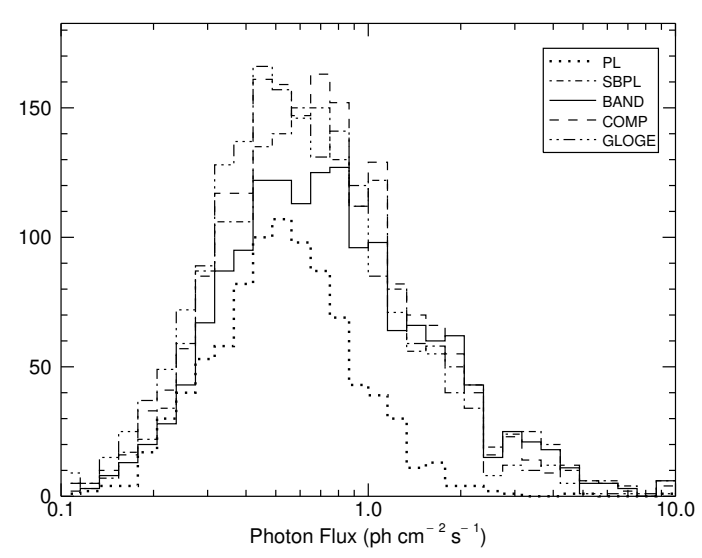

(a)

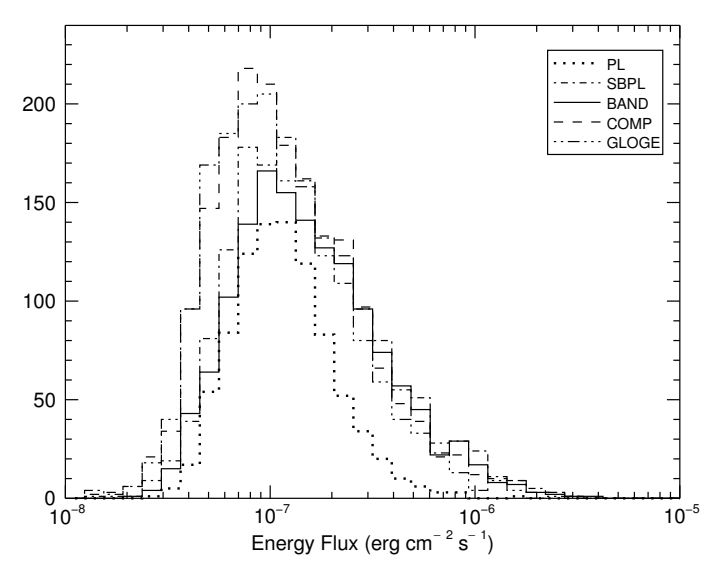

(b)

Fig. 13.- Distributions of photon and energy flux from fluence spectral fits. 13(a) and 13(b) display the flux distributions for the $20 \mathrm{keV}-2 \mathrm{MeV}$ band.

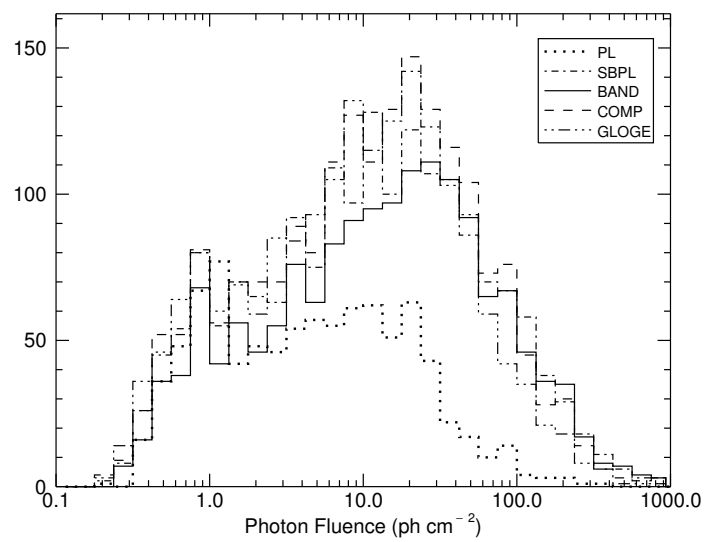

(a)

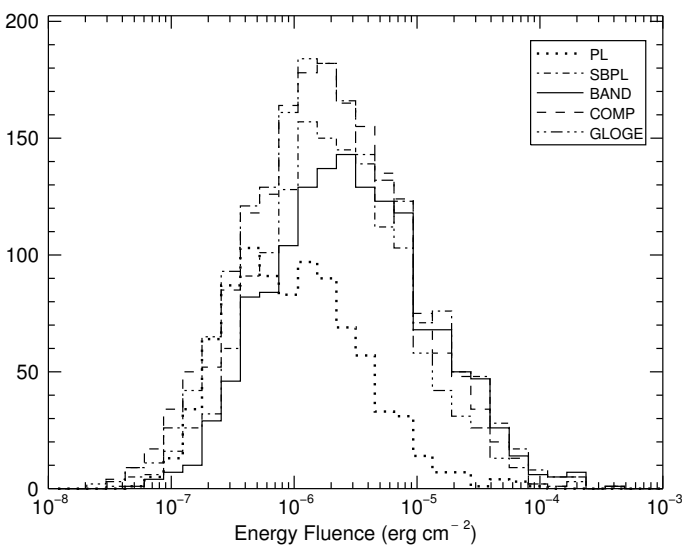

(b)

Fig. 14. - Distributions of photon and energy fluence. 14(a) and 14(b) display the fluence distributions from the $20 \mathrm{keV}-2 \mathrm{MeV}$ band. 


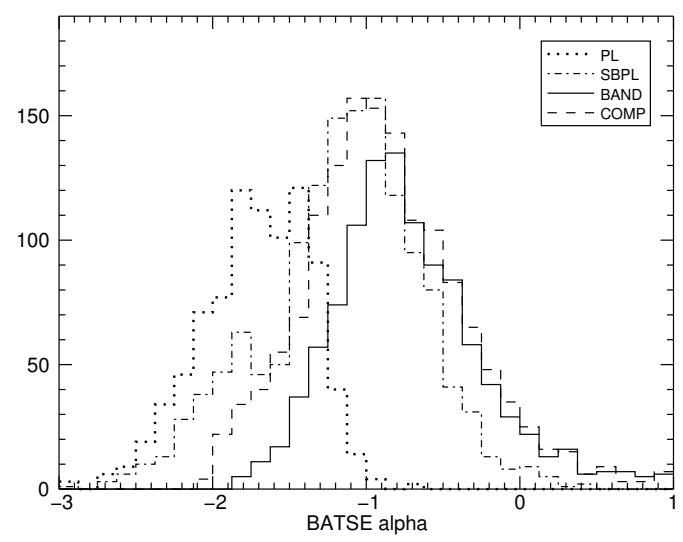

(a)

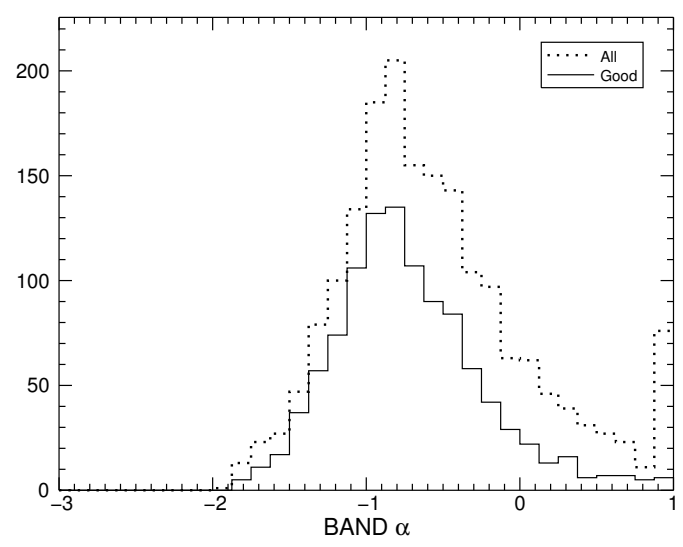

(c)

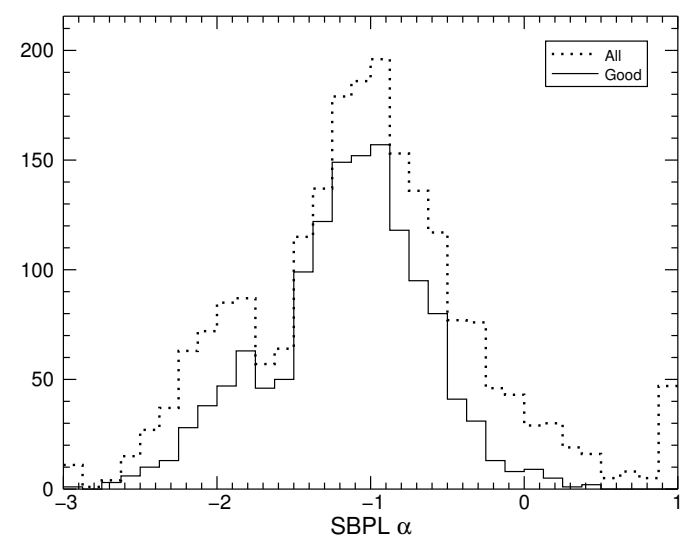

(b)

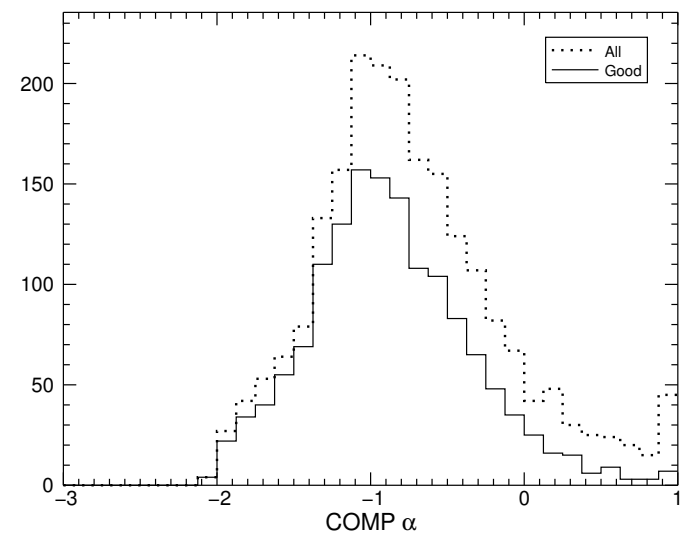

(d)

Fig. 15.- Distributions of the low-energy spectral indices from peak flux spectral fits. 15(a) shows the distributions of GOOD parameters and compares to the distribution of PL indices. 15(b) 15(d) display the comparison between the distribution of GOOD parameters and all parameters with no data cuts. The last bin includes values greater than 1 . 


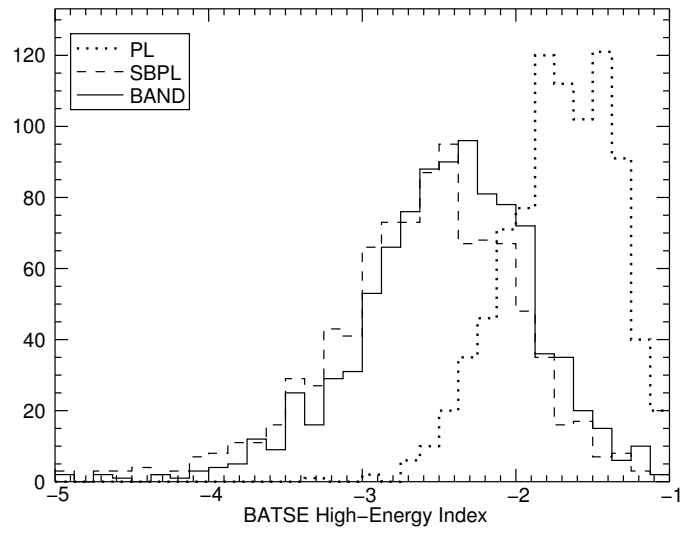

(a)

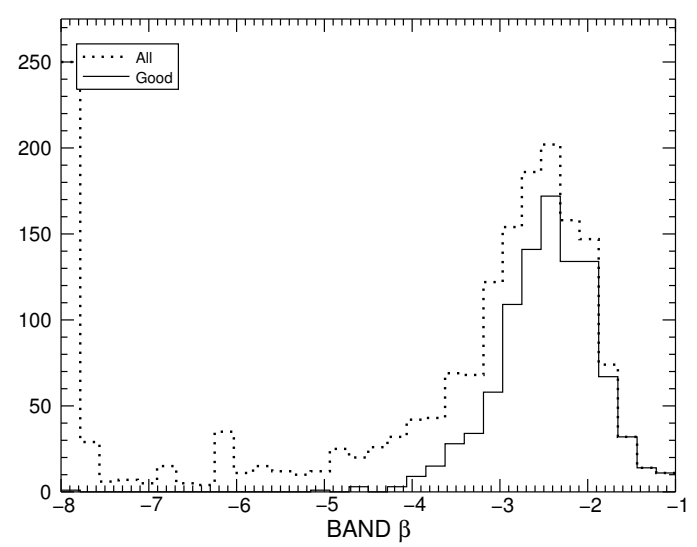

(c)

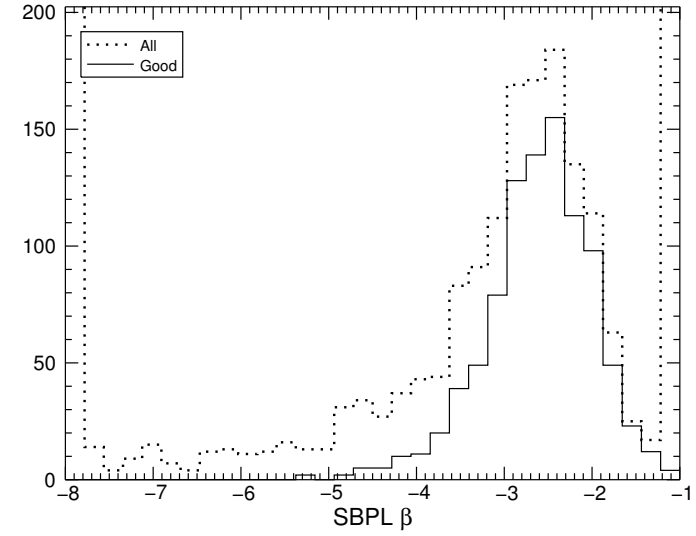

(b)

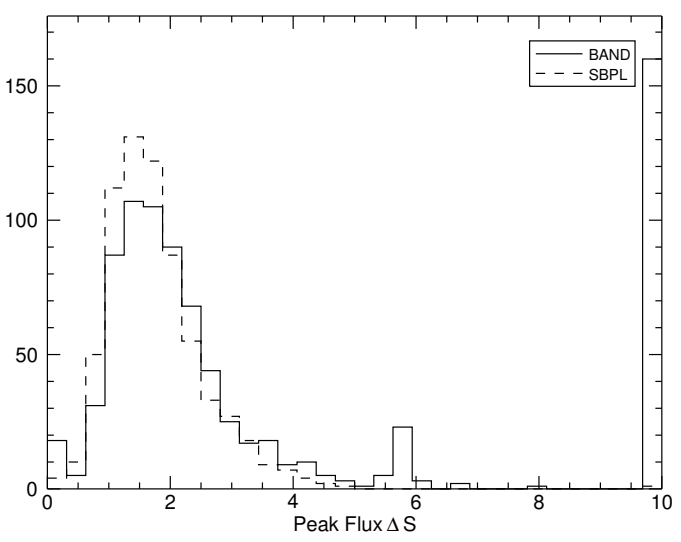

(d)

Fig. 16. - 16(a) - 16(c) are distributions of the high-energy spectral indices from peak flux spectral fits. 16(a) shows the distributions of GOOD parameters and compares to the distribution of PL indices. 16(b) and 16(c) display the comparison between the distribution of GOOD parameters and all parameters with no data cuts. The first bins include values less than -8 and the last bin include values greater than -1 . 16(d) shows the difference between the low- and high-energy indices. The first bin contains values less than 0 , indicating that the centroid value of alpha is steeper than the centroid value of beta. 


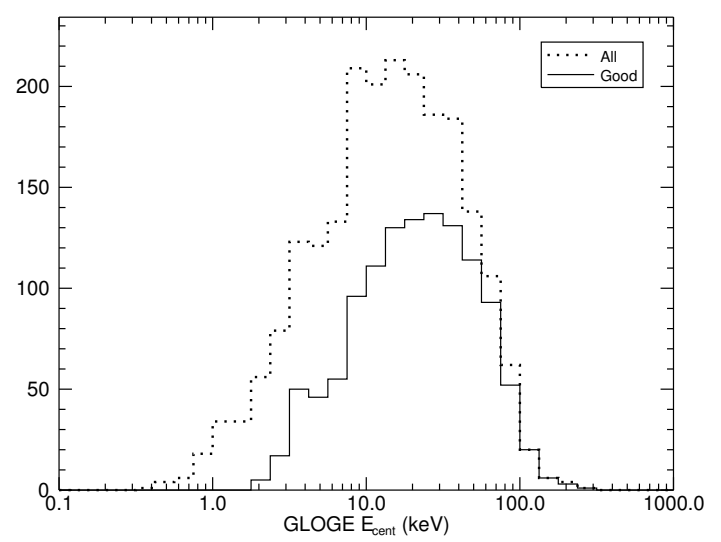

(a)

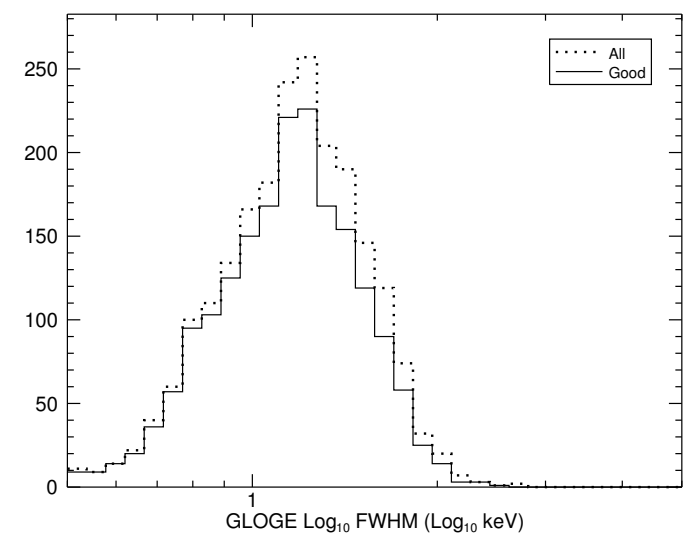

(b)

Fig. 17.- Distributions of the GLOGE $E_{\text {cent }}$ and FWHM parameters from the peak flux spectral fits. 


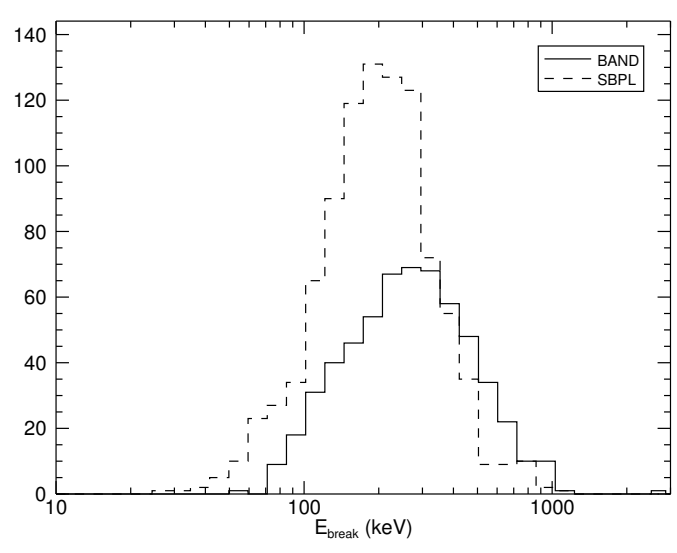

(a)

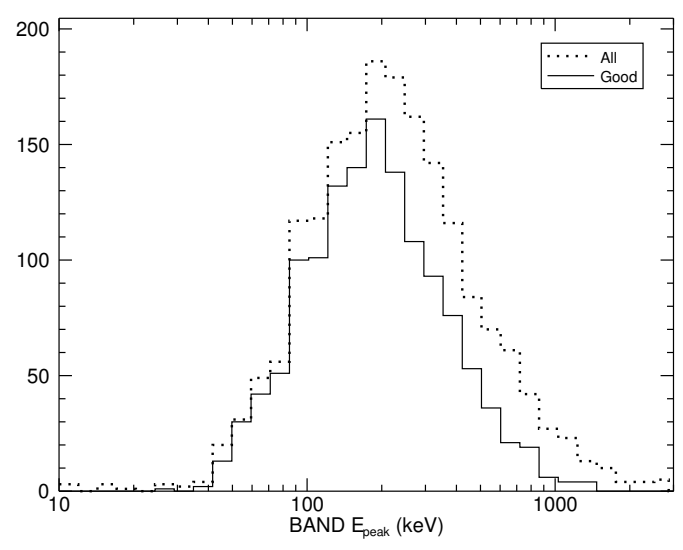

(c)

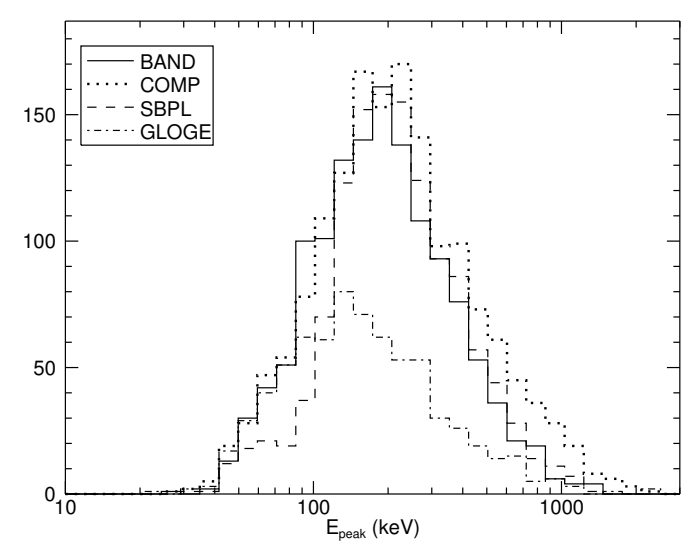

(b)

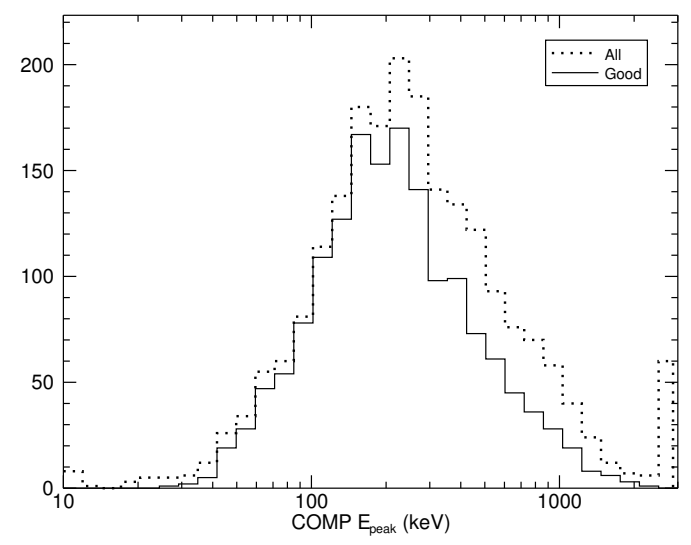

(d)

Fig. 18.- Distributions of $E_{\text {break }}$ and $E_{\text {peak }}$ from peak flux spectral fits. 18(a) displays the comparison between the distribution of GOOD $E_{\text {break }}$ and $E_{\text {break }}$ with no data cuts. 18(b) shows the distributions of GOOD $E_{\text {peak }}$ for BAND, SBPL, and COMP. 18(c) and 18(d) display the comparison between the distribution of GOOD parameters and all parameters with no data cuts. 


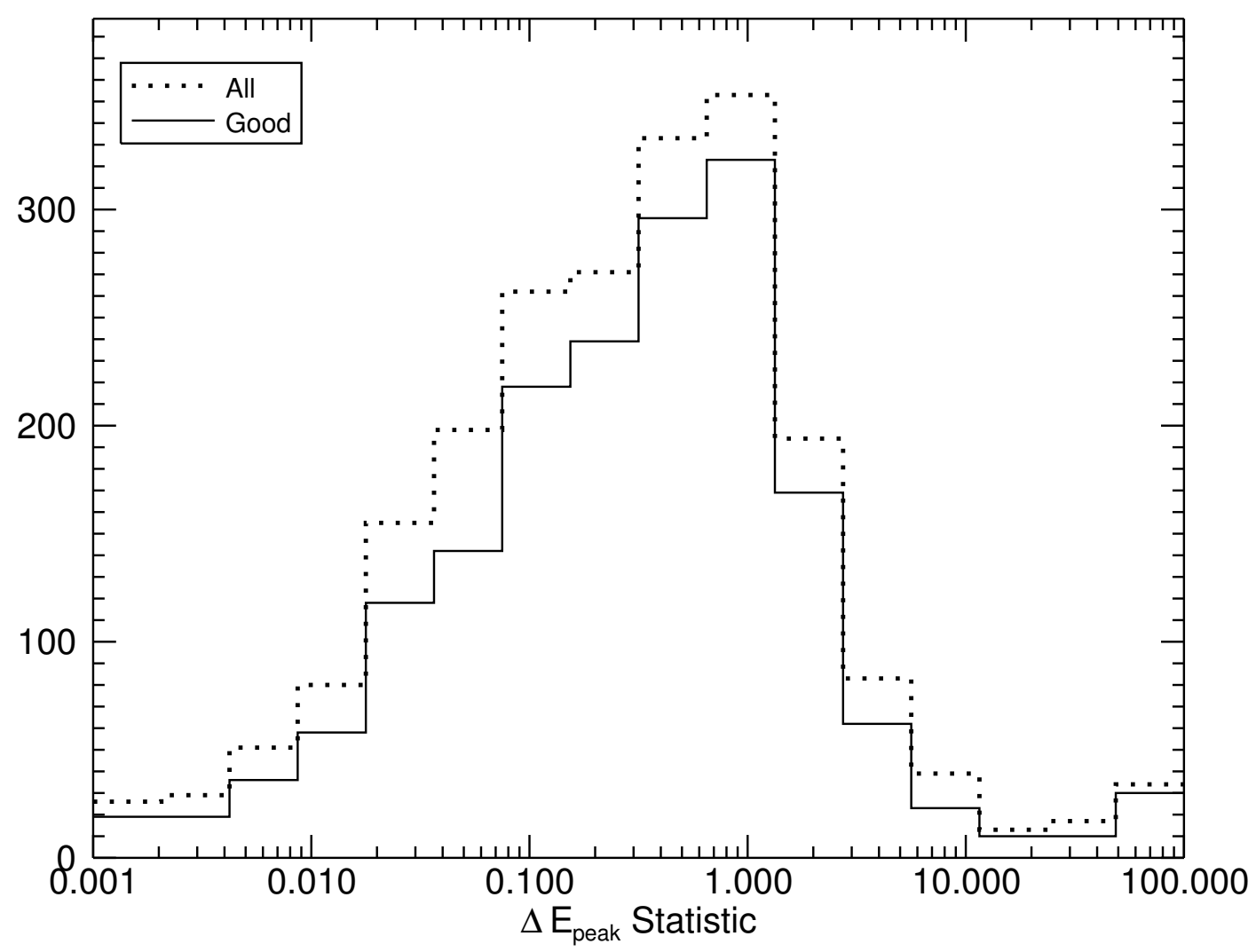

Fig. 19.- Distribution of the $\Delta E_{\text {peak }}$ statistic for the COMP and BAND models from peak flux spectral fits. A value less than 1 indicates the $E_{\text {peak }}$ values are similar within errors, while a value larger than 1 indicates the $E_{\text {peak }}$ values are not within errors. 


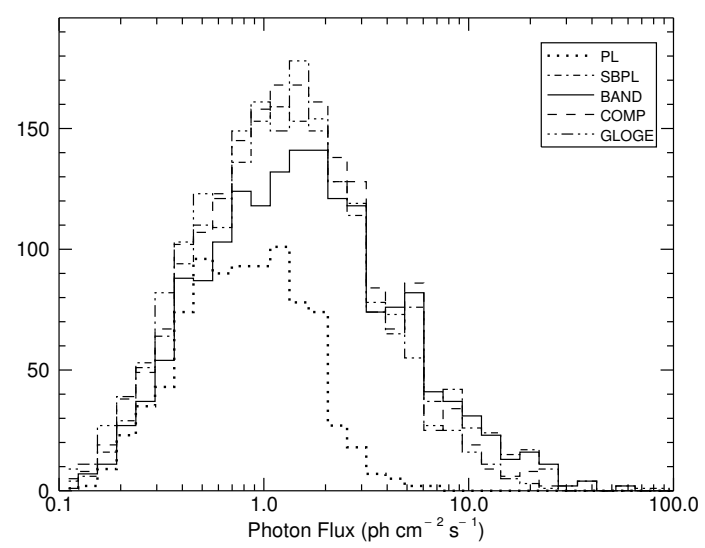

(a)

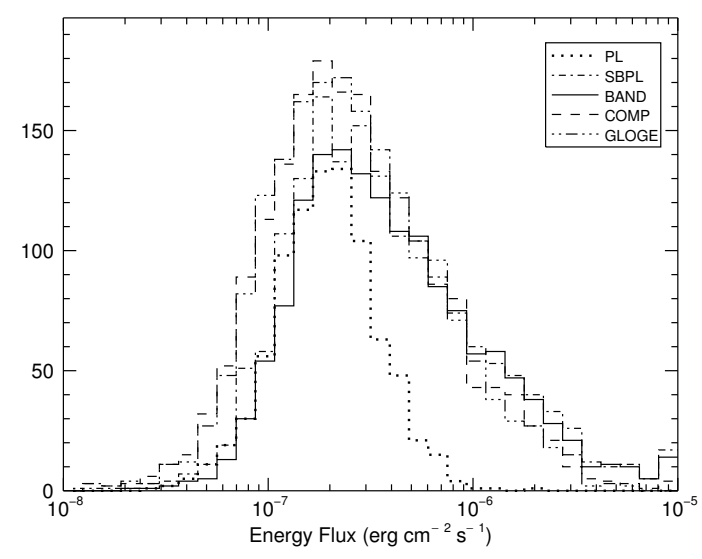

(b)

Fig. 20.- Distributions of photon and energy flux from peak flux spectral fits. 20(a) and 20(b) display the flux distributions for the $20 \mathrm{keV}-2 \mathrm{MeV}$ band. 


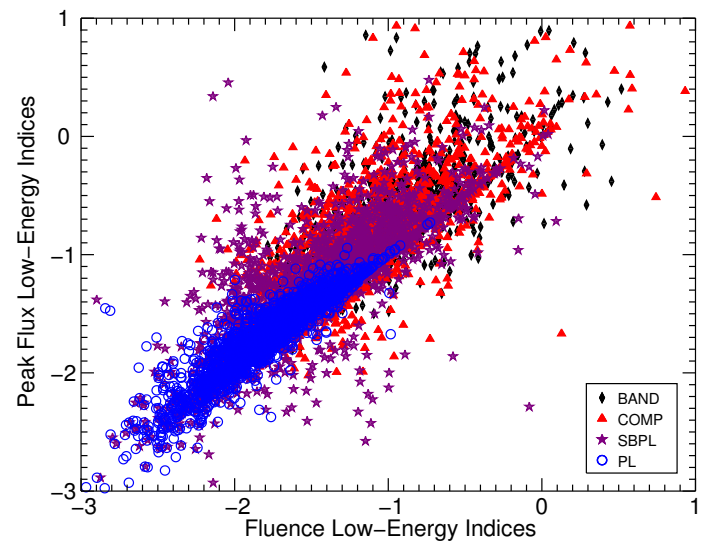

(a)

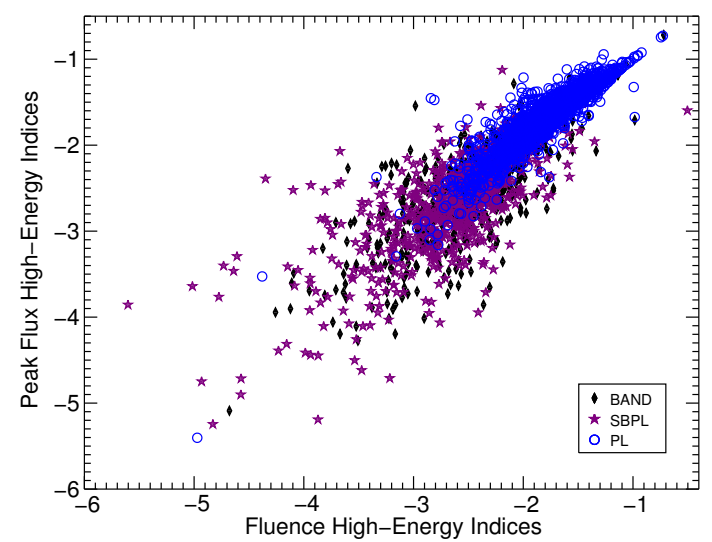

(b)

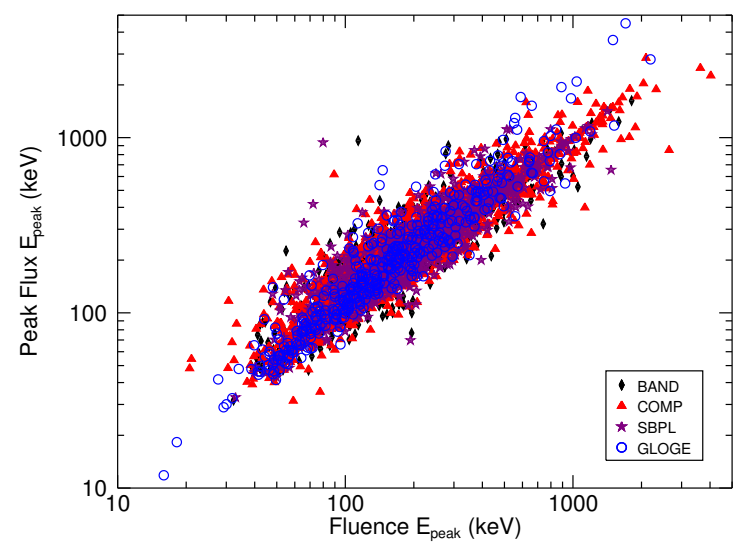

(c)

Fig. 21. - Peak flux spectral parameters as a function of the fluence spectral parameters. For all three parameters there is evidence for a strong correlation between the parameters found for the fluence spectra and those for the peak flux spectra. Note that the PL index is shown in both 21(a) and 21(b) for comparison. 


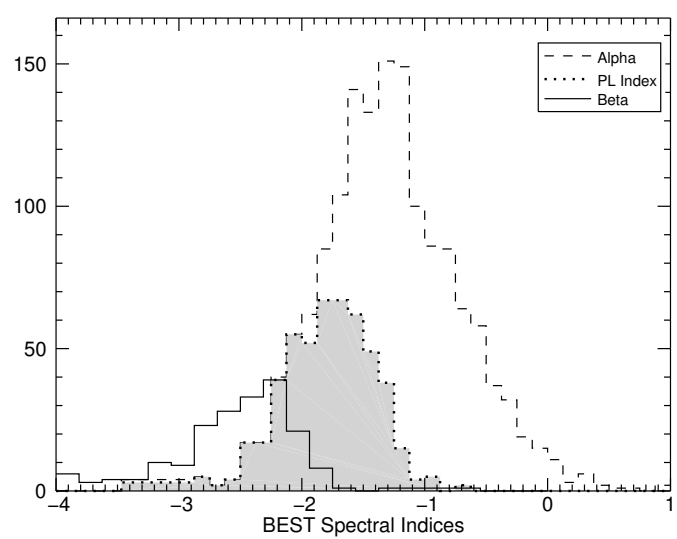

(a)

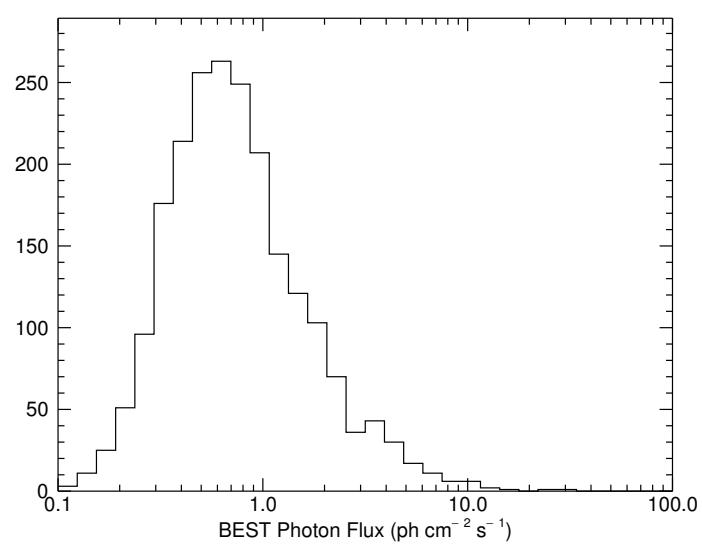

(c)

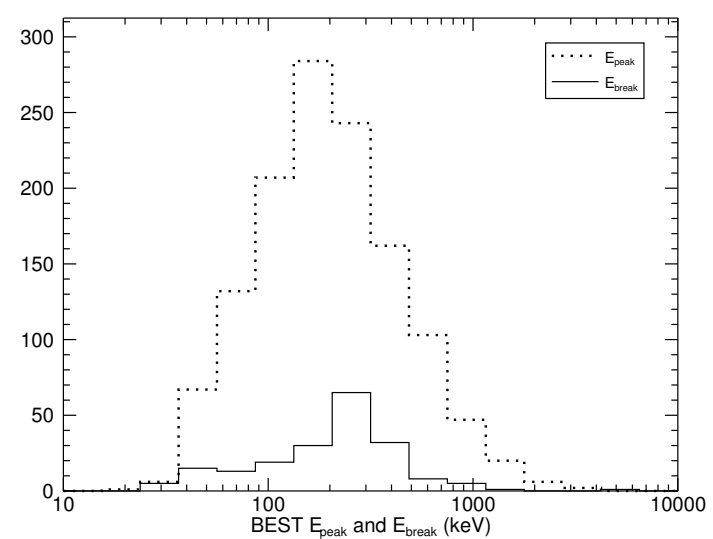

(b)

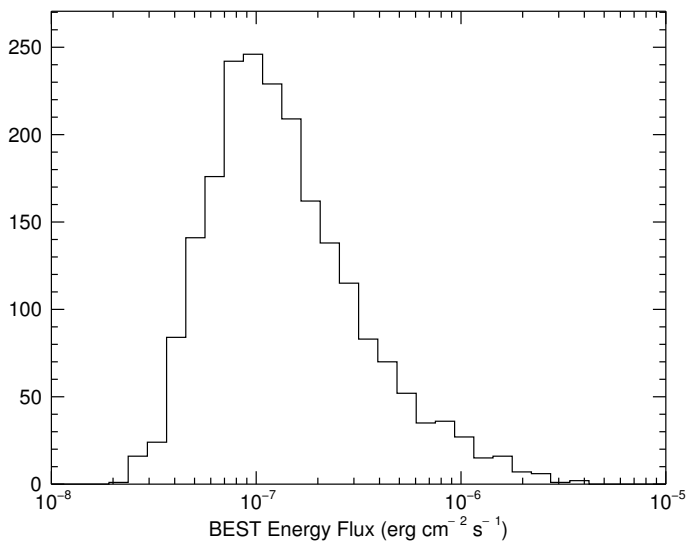

(d)

Fig. 22.- Distributions of the BEST spectral parameters for the fluence spectra. 22(a) displays the selection of best low-energy and high-energy spectral indices. The shaded distribution depicts the location of the distribution of the PL index. 22(b)] shows the selection of the best $E_{\text {peak }}$ and $E_{\text {break }} \cdot 22(\mathrm{c})$ and $22(\mathrm{~d})$ show the selection of the best photon flux and energy flux respectively. 


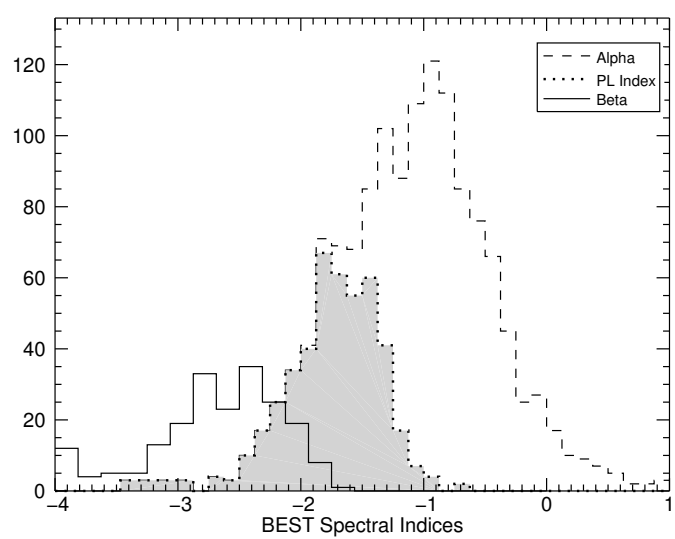

(a)

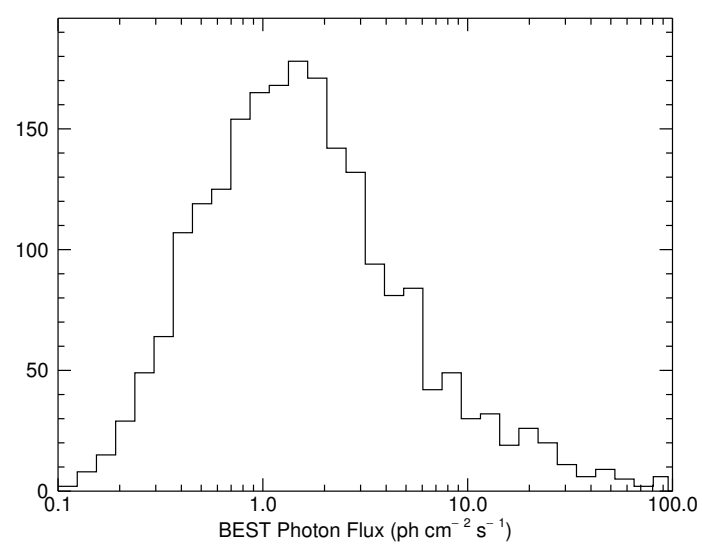

(c)

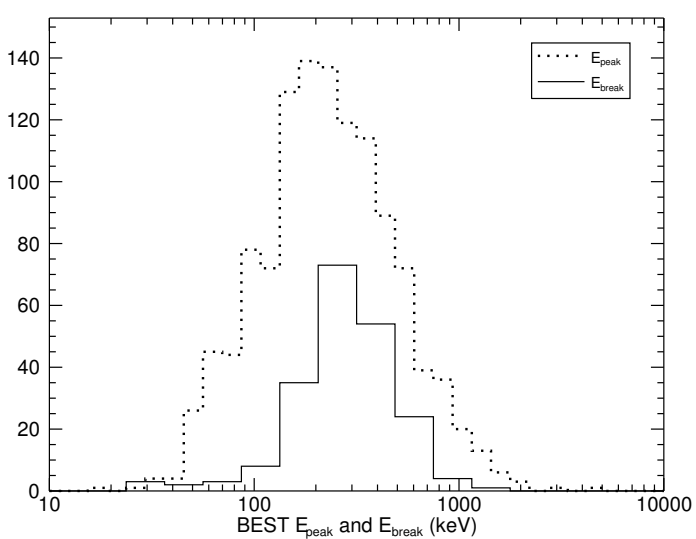

(b)

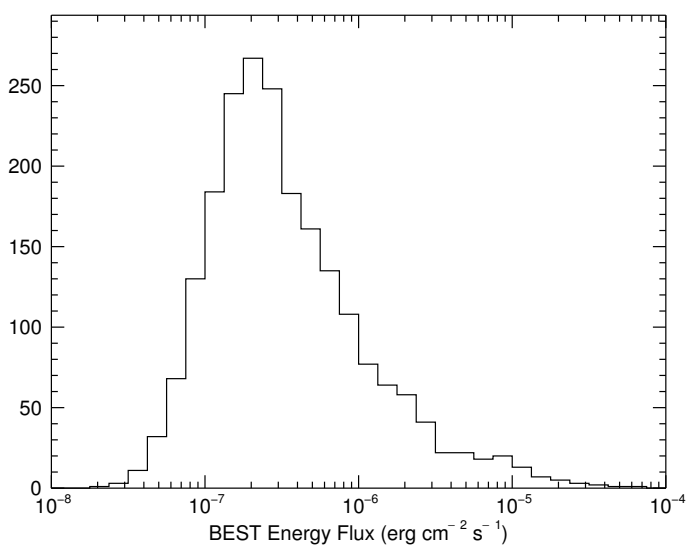

(d)

Fig. 23.- Distributions of the BEST spectral parameters for the peak flux spectra. 23(a) displays the selection of best low-energy and high-energy spectral indices. The shaded distribution depicts the location of the distribution of the PL index. 23(b) shows the selection of the best $E_{\text {peak }}$ and $E_{\text {break }}$. 23(c) and 23(d) show the selection of the best photon flux and energy flux respectively. 


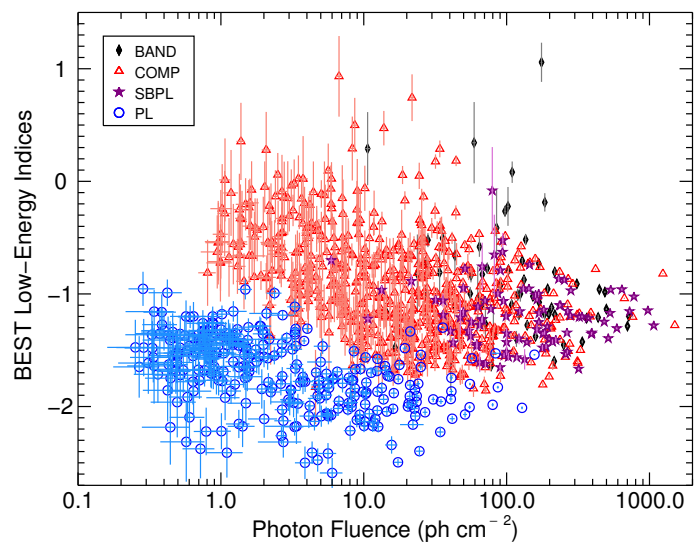

(a)

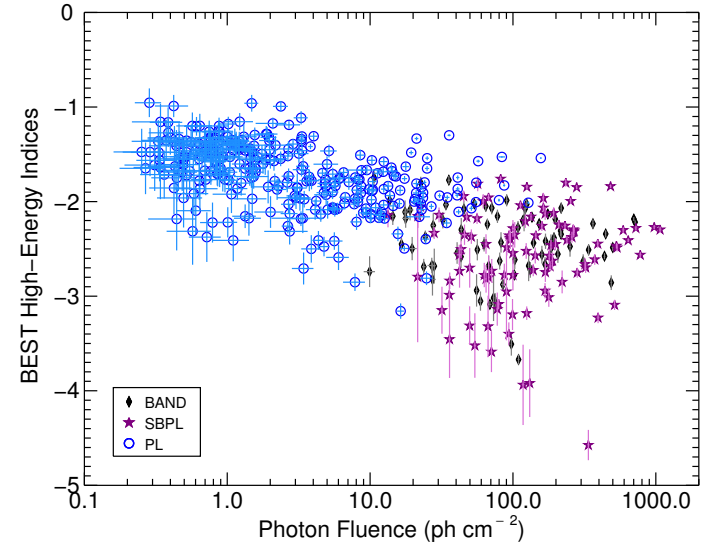

(b)

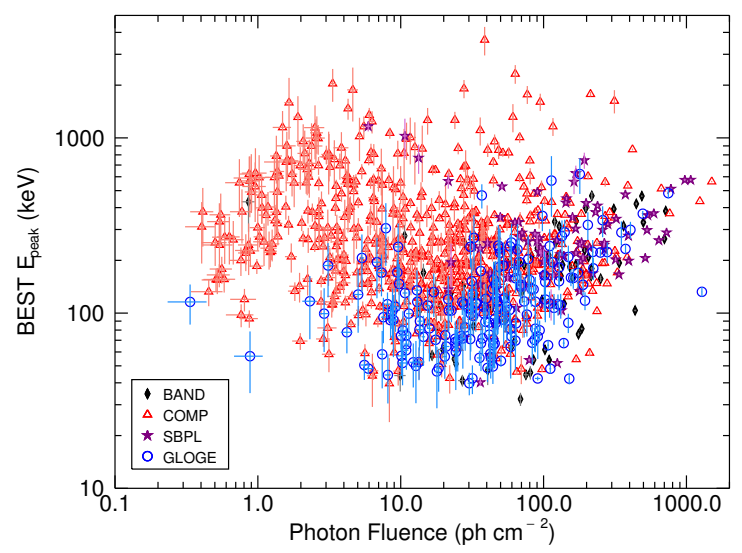

(c)

Fig. 24.- BEST fluence spectral parameters as a function of the model photon fluence. Note that the PL index is shown in both 24(a) and 24(b) for comparison. 


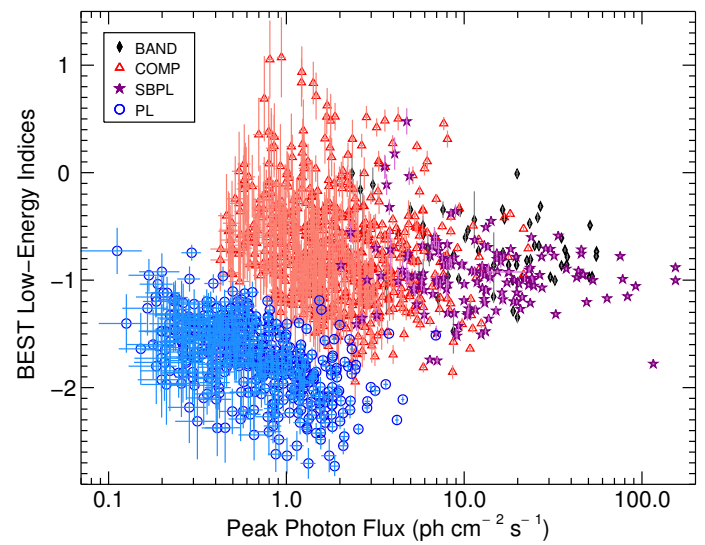

(a)

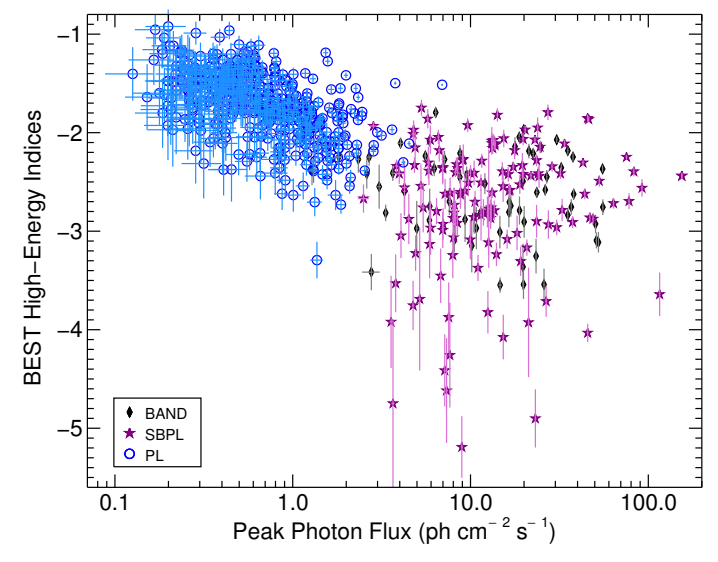

(b)

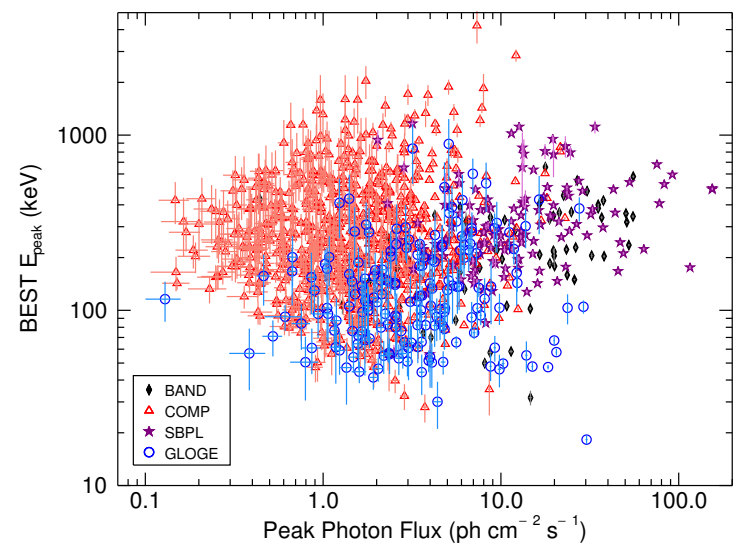

(c)

Fig. 25.- BEST peak flux spectral parameters as a function of the model peak photon flux. Note that the PL index is shown in both 25(a) and 25(b) for comparison. 


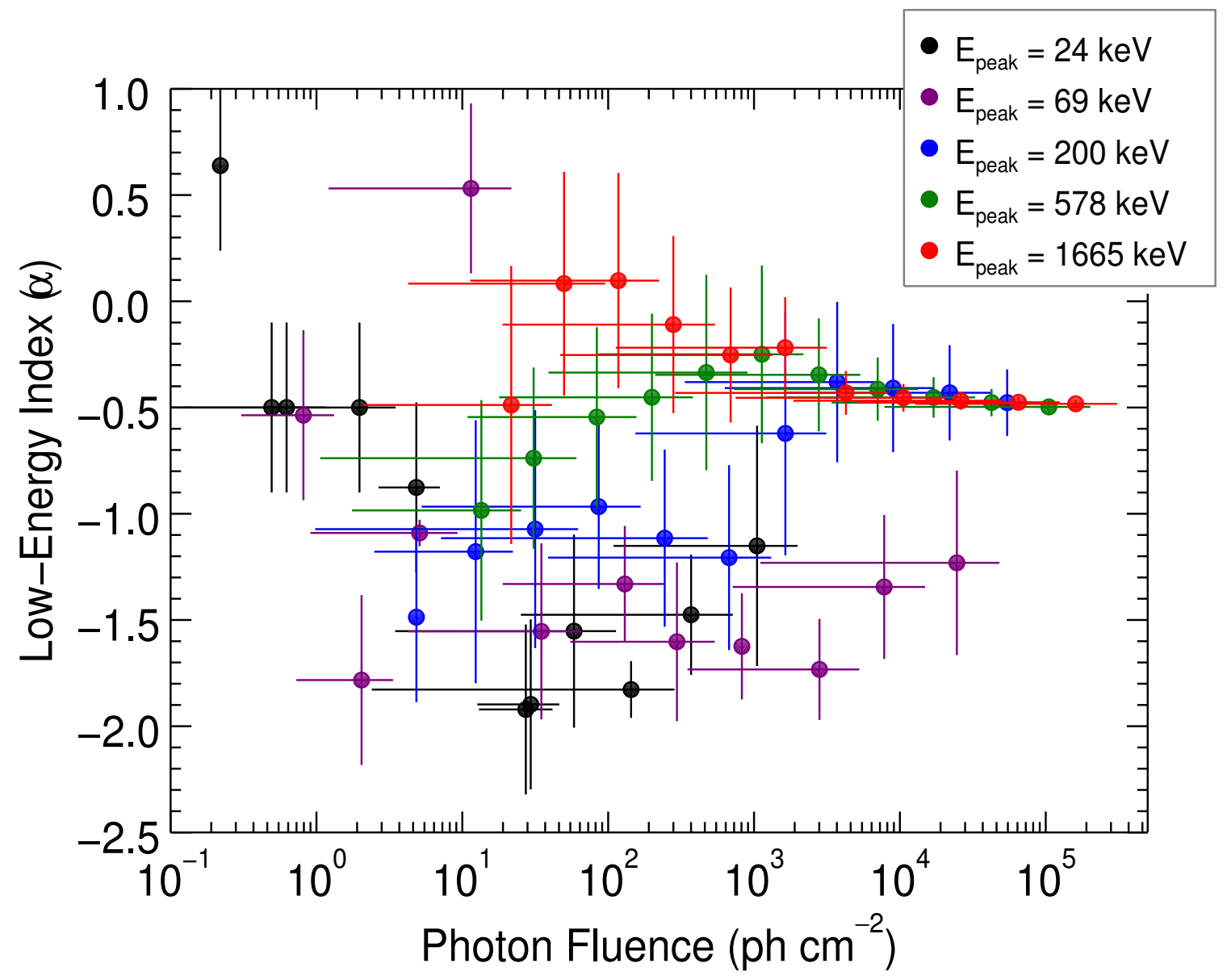

Fig. 26.- Simulations of fitting the BAND low-energy index (alpha) as a function of the spectral photon fluence for five different values of $E_{\text {peak }}$. 


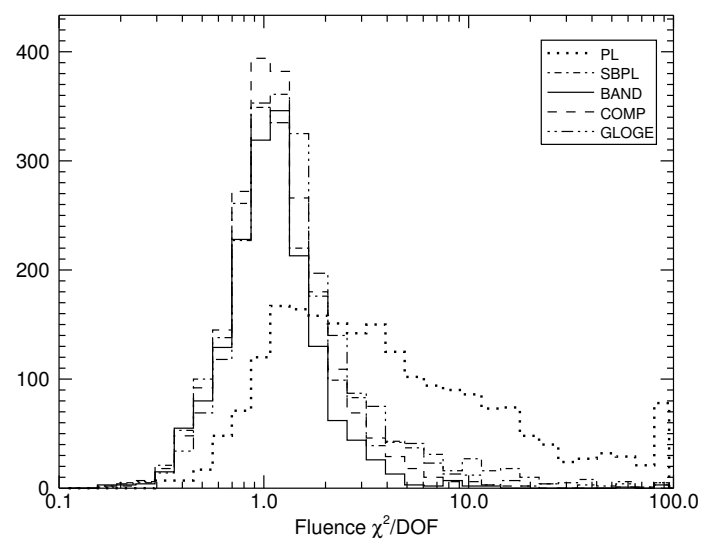

(a)

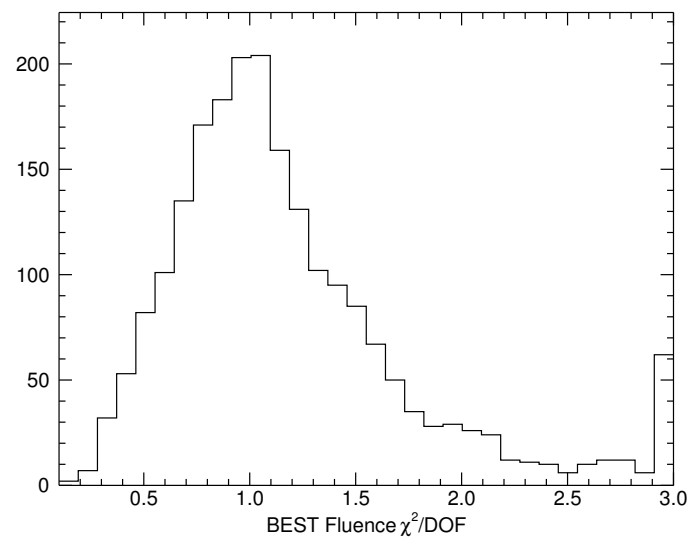

(c)

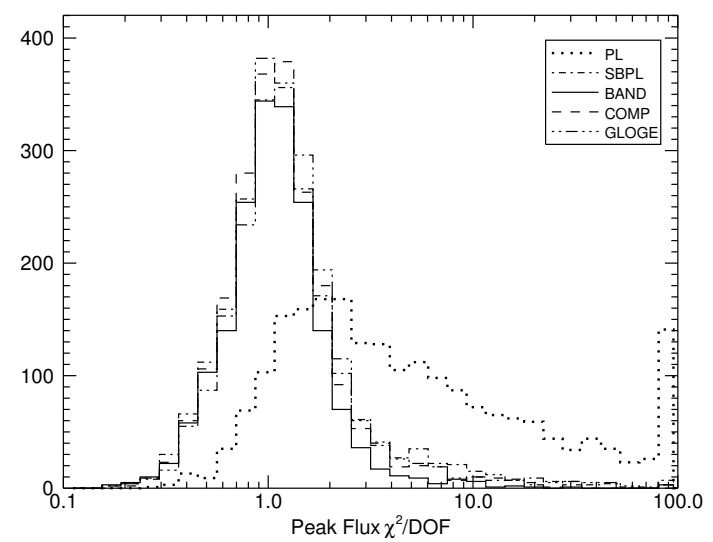

(b)

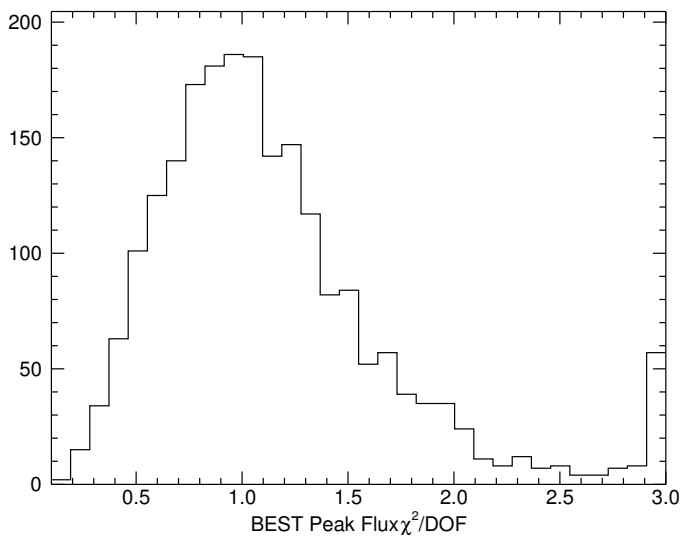

(d)

Fig. 27.- Distributions of the reduced $\chi^{2}$ for each model. 27(a) and 27(b) are distributions of the reduced $\chi^{2}$ for each model for each burst. Note that these distributions include all fits, including unconstrained fits. 27(c) and 27(d) show the distribution of BEST reduced $\chi^{2}$ values. The first and last bins in all distributions contain overflow values. 


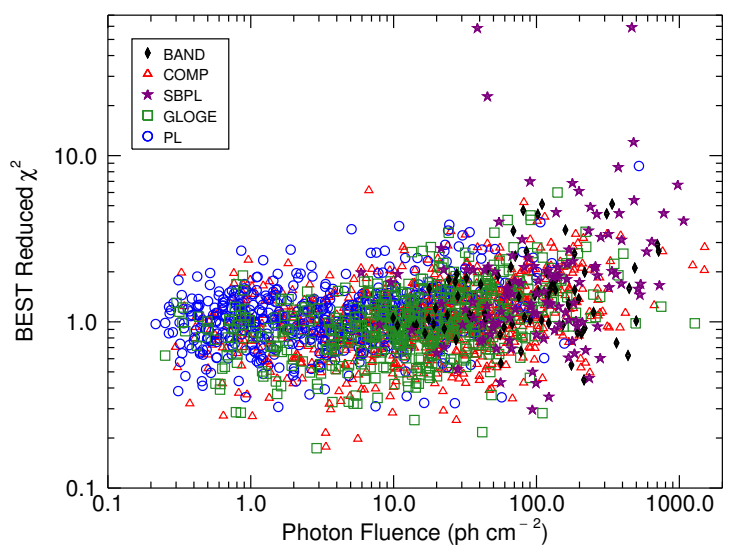

(a)

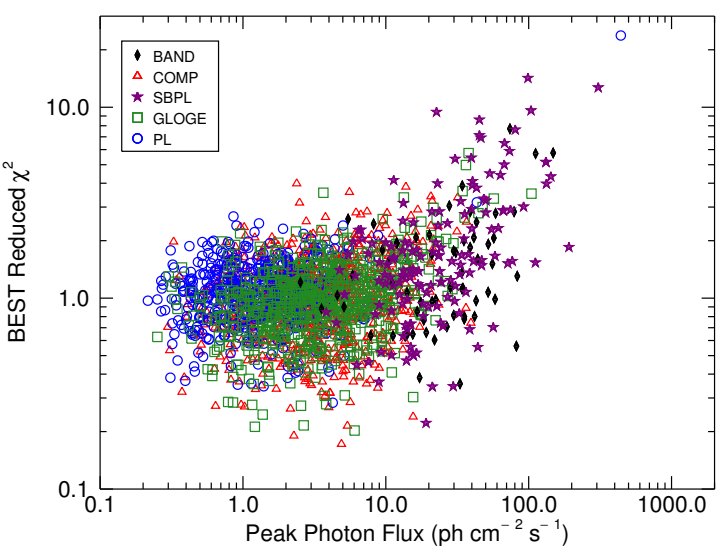

(b)

Fig. 28.- 28(a) Reduced $\chi^{2}$ as a function of the model photon fluence. 28(b) Reduced $\chi^{2}$ as a function of the model peak photon flux. 


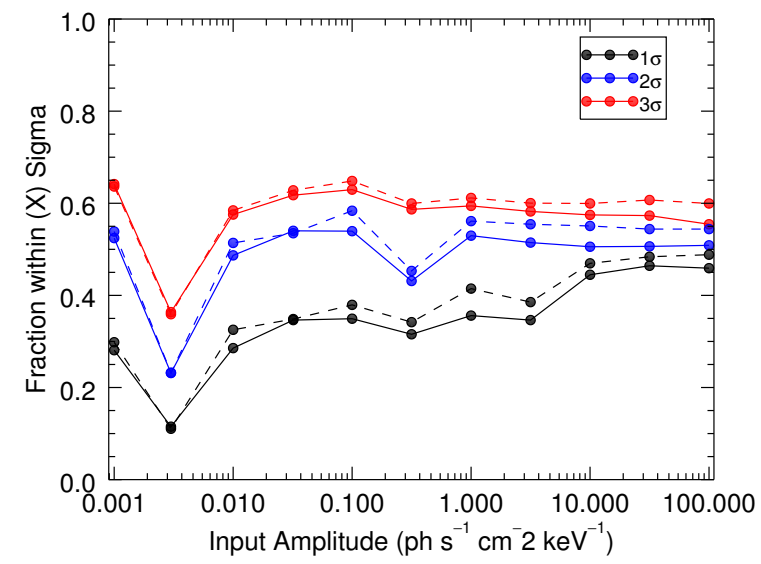

(a)

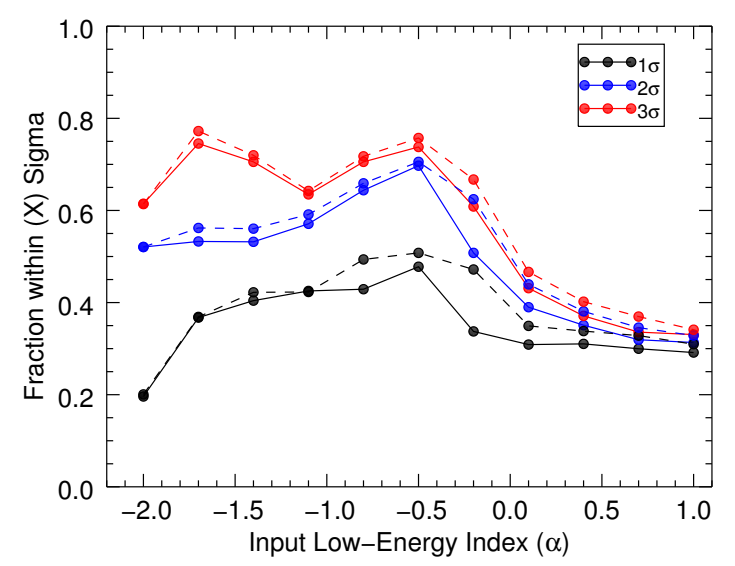

(c)

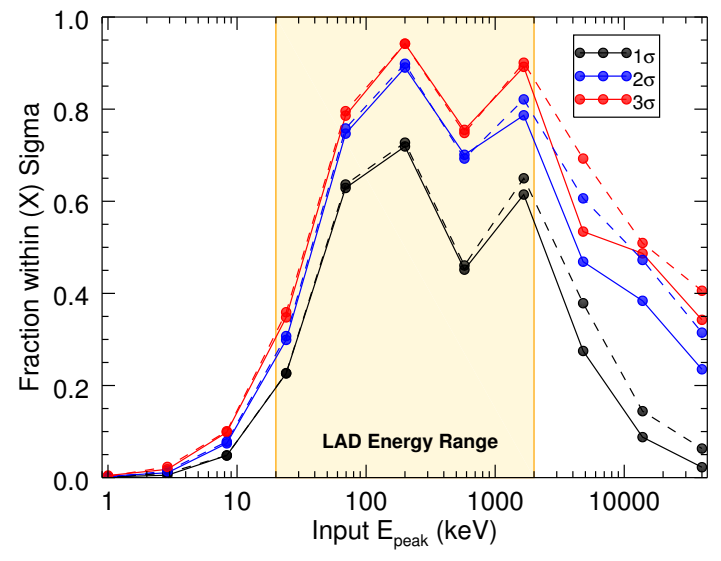

(b)

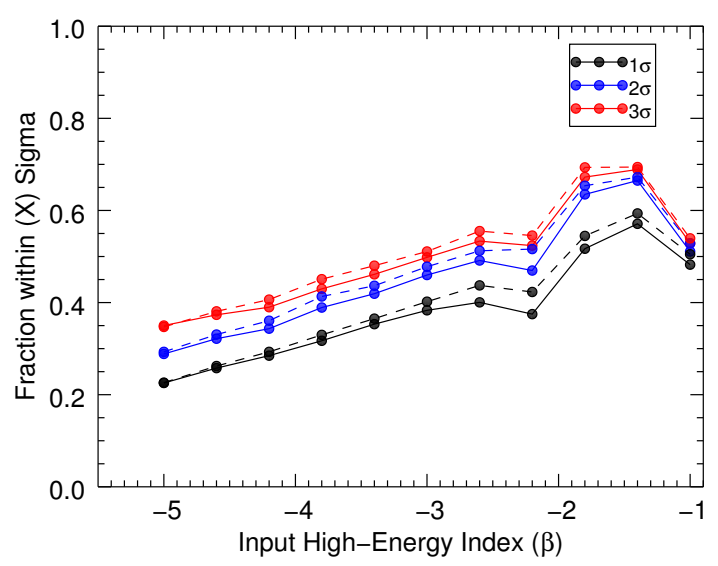

(d)

Fig. 29. - Plots of the effectiveness of the BATSE LADs when fitting a Band spectrum. The solid line signifies the 16-channel CONT data, while the dashed line represents the 128Channel HERB data. The three colors signify the distance in standard deviations between the mean value of the parameter from the spectral fits and the input spectral values. The standard deviation for each parameter is defined by the $68 \%$ confidence interval resulting from each set of simulations. 


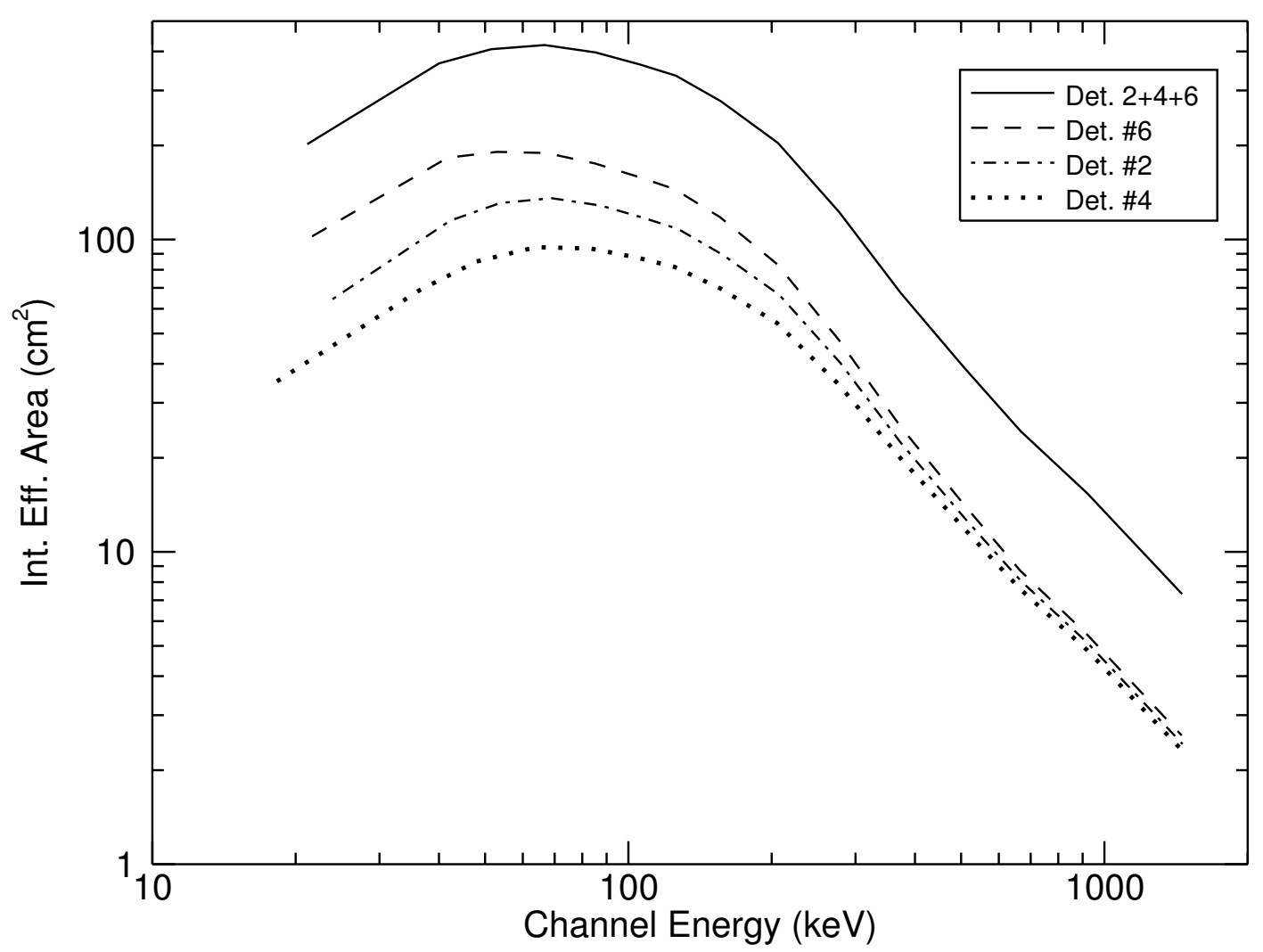

Fig. 30.- Plot of the effective area integrated over photon energies of BATSE detectors for GRB 910503 (trigger \#0143). Each detector possesses its own response that is dependent on angle and energy. Adding these responses together effectively averages the features of the individual responses and spectral resolution is decreased. 


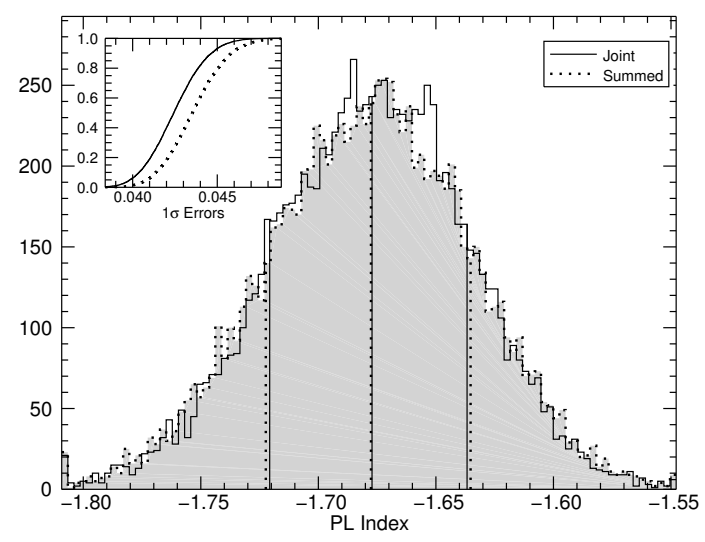

(a)

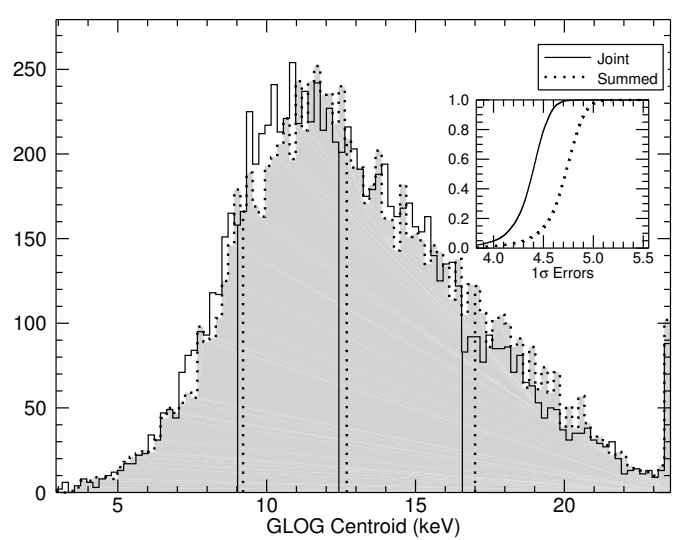

(b)

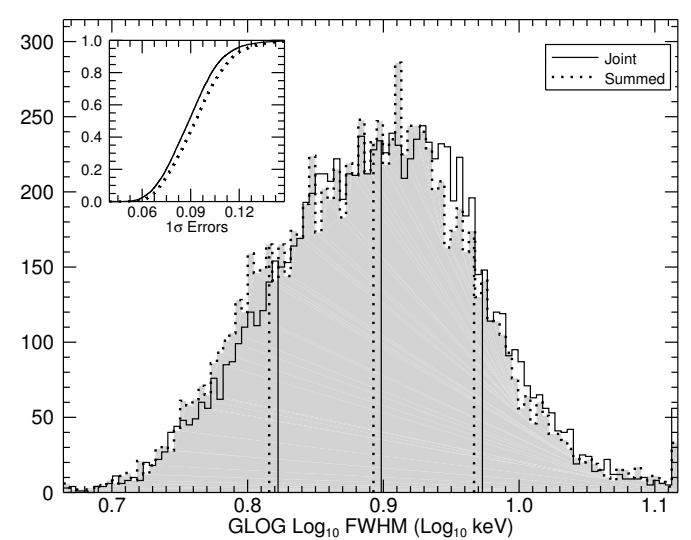

(c)

Fig. 31. - Simulations of PL 31(a) and GLOGE 31(b) \& 31(c) parameters. 31(a) compares the summed detectors with the joint detectors for GRB 910425 (BATSE Trigger \#0110) and 31(b) \& 31(c) shows the same comparison for GRB 910521 (BATSE Trigger \#0214). The shaded histograms represent the parameter distributions for the summed detectors, and the empty histograms represent the joint detectors. The central vertical lines represent the sample mean, while the vertical lines on either side of the mean represent the asymmetric sample standard deviation. The inset plots show the cumulative distributions of the parameter errors. 


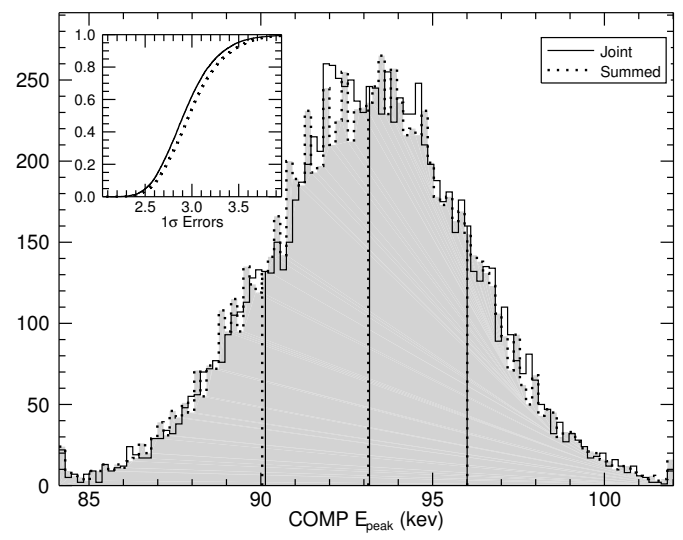

(a)

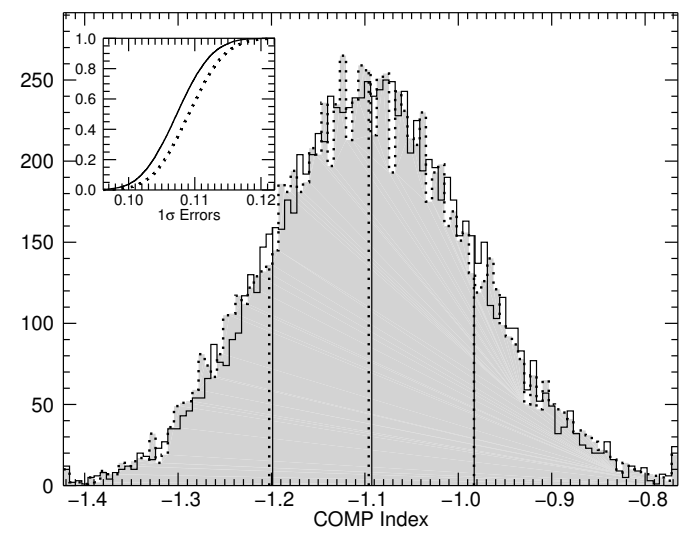

(b)

Fig. 32.- Simulations of COMP parameters. The plots compare the summed detectors with the joint detectors for GRB 910426 (BATSE Trigger \#0111). The shaded histograms represent the parameter distributions for the summed detectors, and the empty histograms represent the joint detectors. The central vertical lines represent the sample mean, while the vertical lines on either side of the mean represent the asymmetric sample standard deviation. The inset plots show the cumulative distributions of the parameter errors. 


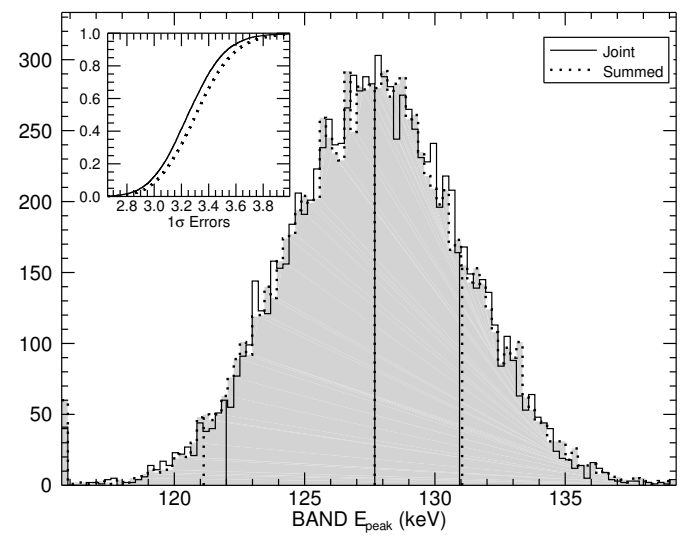

(a)

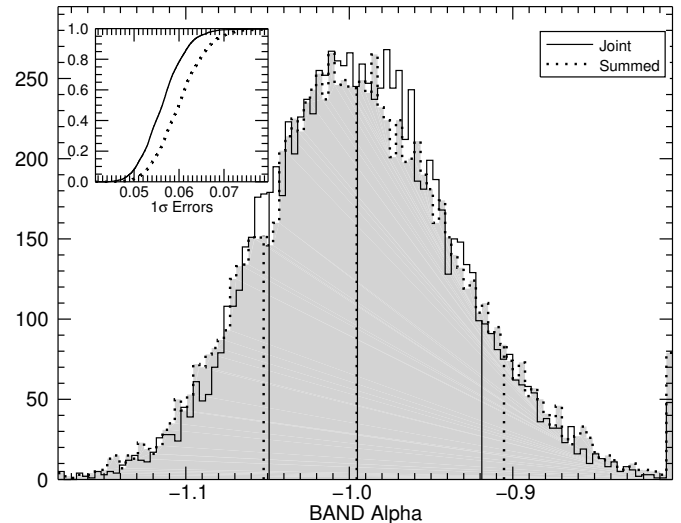

(b)

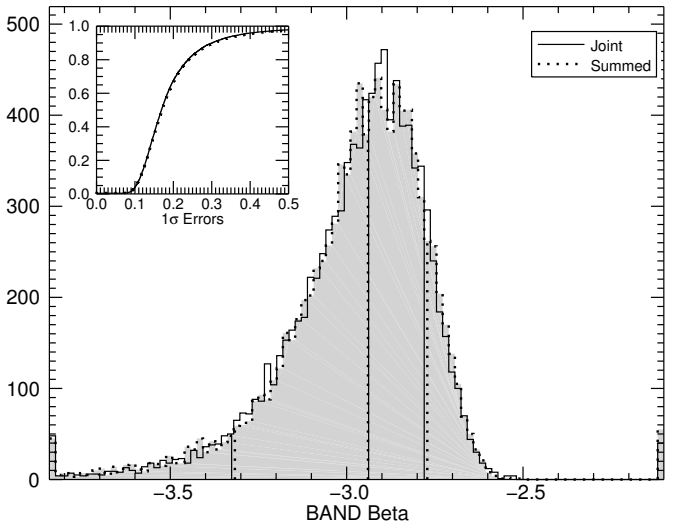

(c)

Fig. 33. - Simulations of BAND parameters. The plots compare the summed detectors with the joint detectors for GRB 910421 (BATSE Trigger \#0105). The shaded histograms represent the parameter distributions for the summed detectors, and the empty histograms represent the joint detectors. The central vertical lines represent the sample mean, while the vertical lines on either side of the mean represent the asymmetric sample standard deviation. The inset plots show the cumulative distributions of the parameter errors. 


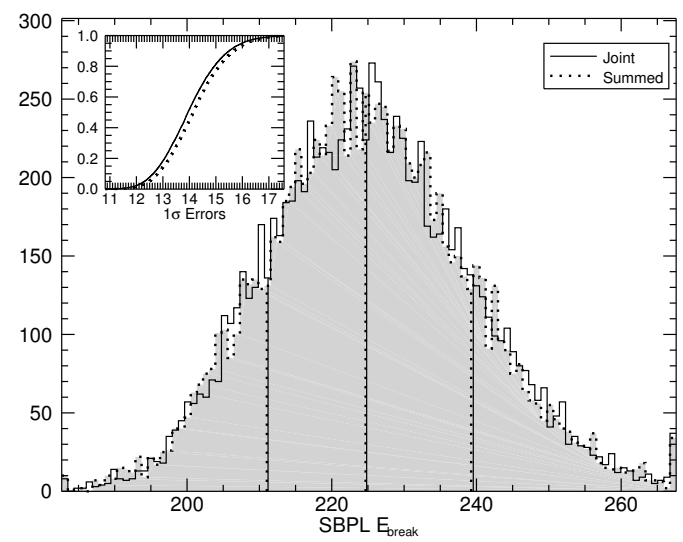

(a)

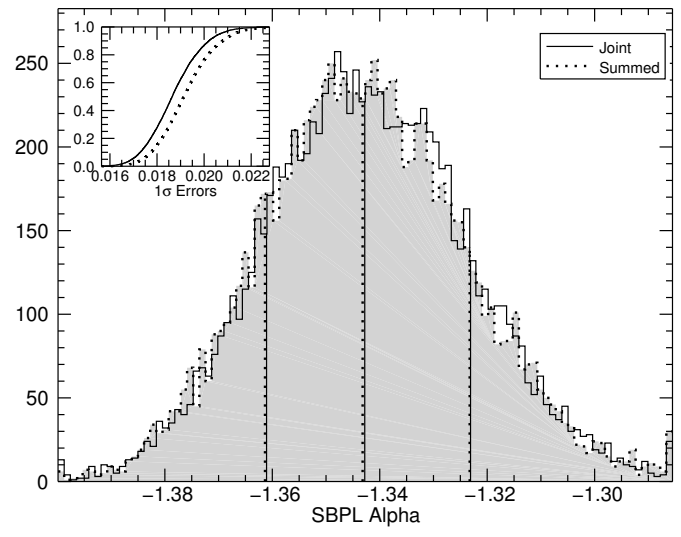

(b)

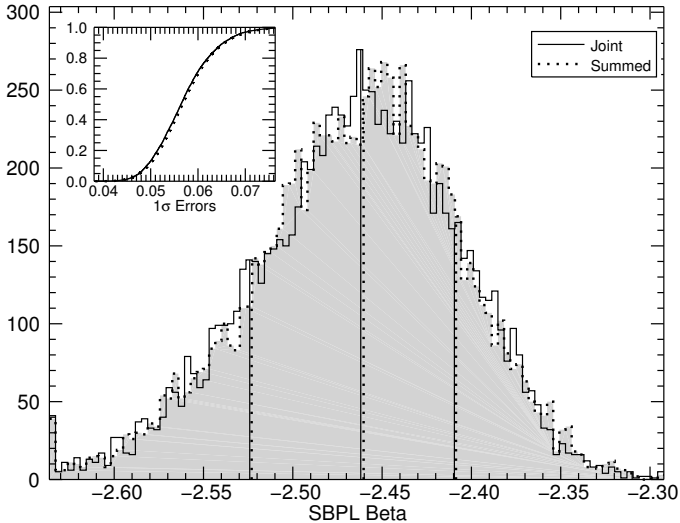

(c)

Fig. 34.- Simulations of SBPL parameters. The plots compare the summed detectors with the joint detectors for GRB 910522 (BATSE Trigger \#0219). The shaded histograms represent the parameter distributions for the summed detectors, and the empty histograms represent the joint detectors. The central vertical lines represent the sample mean, while the vertical lines on either side of the mean represent the asymmetric sample standard deviation. The inset plots show the cumulative distributions of the parameter errors. 


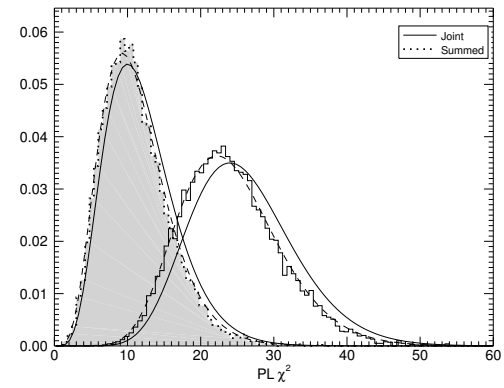

(a)

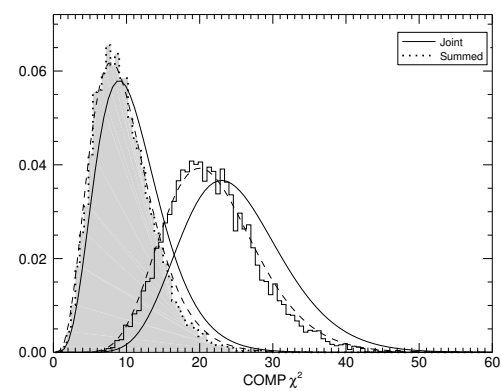

(c)

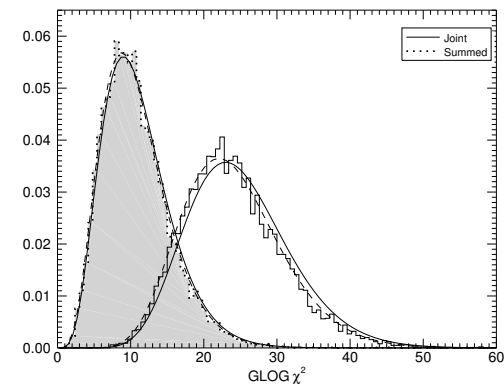

(b)

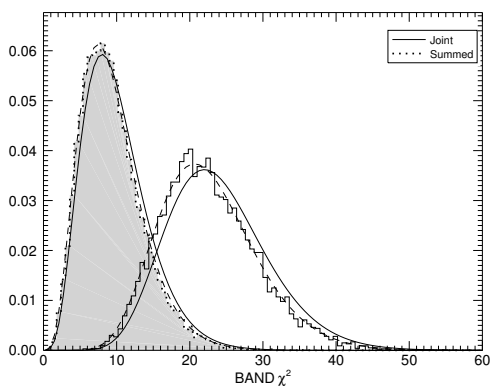

(d)

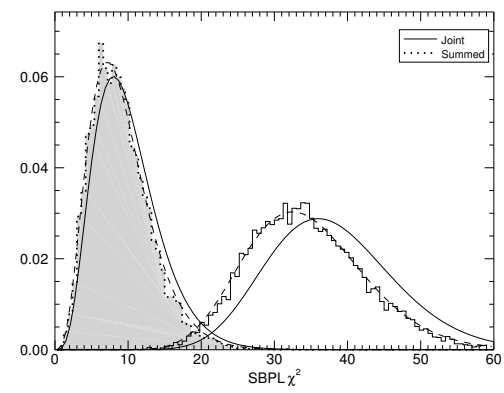

(e)

Fig. 35.- Comparisons of the $\chi^{2}$ distributions between the joint and summed simulations. The solid curve represents the $\chi^{2}$ distribution for the defined number of degrees of freedom. The dashed curve is a best fit $\chi^{2}$ distribution showing that the distribution of $\chi^{2}$ values from both simulations are shifted from what is expected, although by no more than $10 \%$ in any one case. Both the summed and joint distributions are shifted by roughly the same amount, therefore, the shift is not due to summing detectors. 


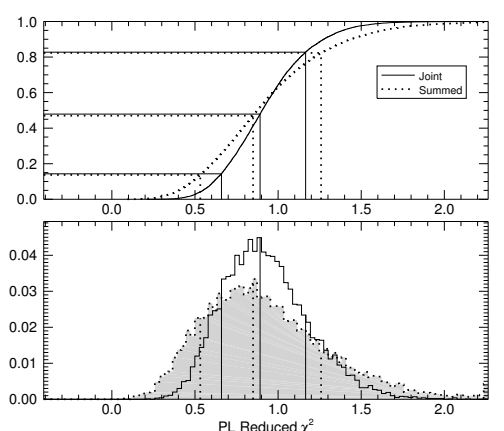

(a)
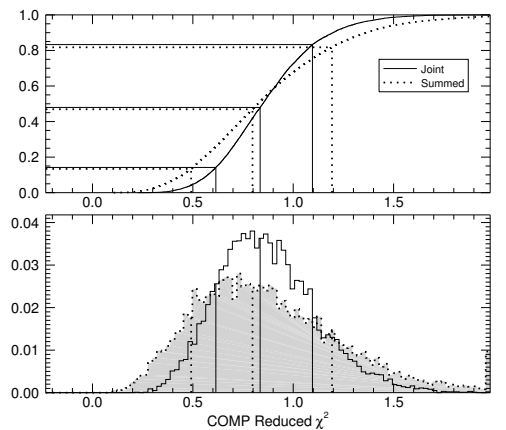

(c)

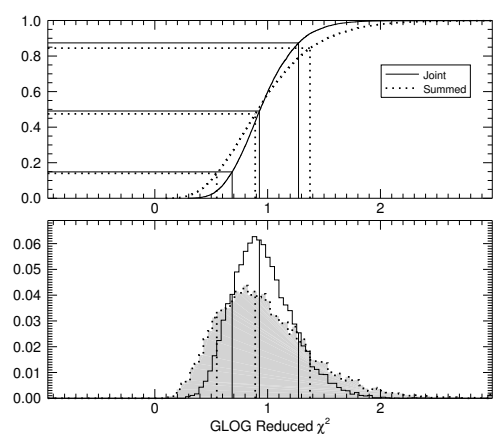

(b)

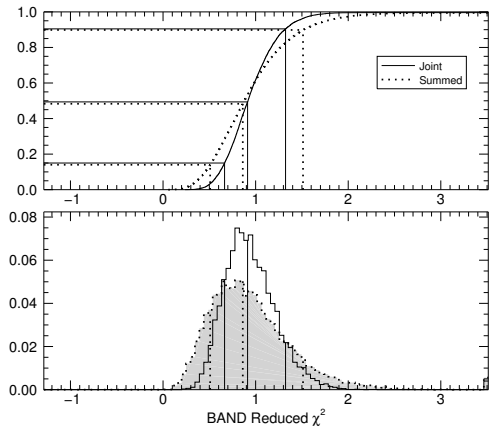

(d)

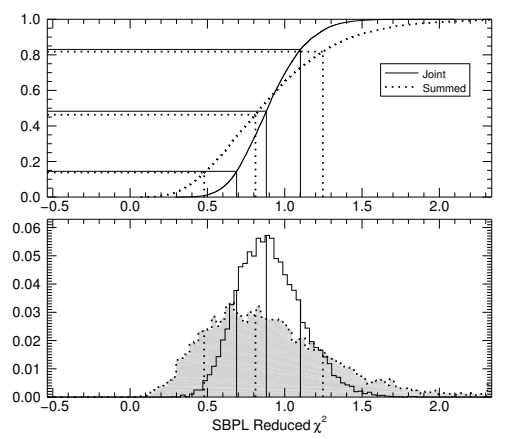

(e)

Fig. 36.- Comparisons of the reduced $\chi^{2}$ distributions between the joint and summed simulations. The upper plots show the cumulative distributions and reveal the large differences in the variance of the distributions shown in the lower plots. The three vertical lines in each plot represent the sample means (middle line), and the bounds of the sample standard deviations. In each case the means between the summed and joint simulations are approximately the same, yet the variance for the summed simulations are considerably larger than that for the joint simulations. 


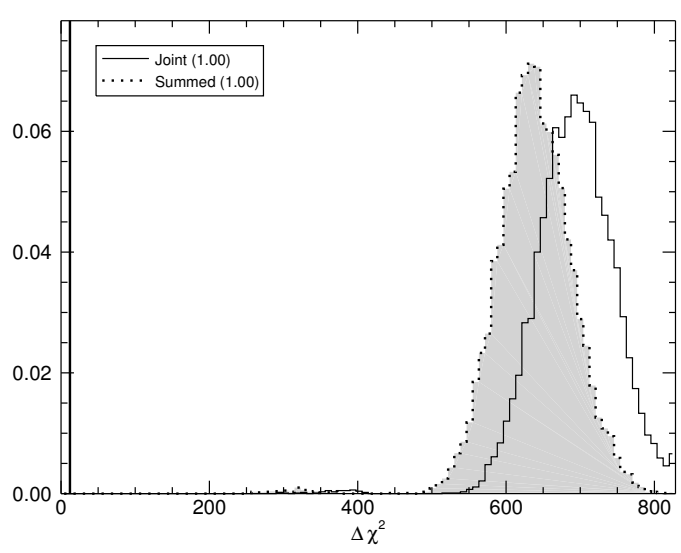

(a)

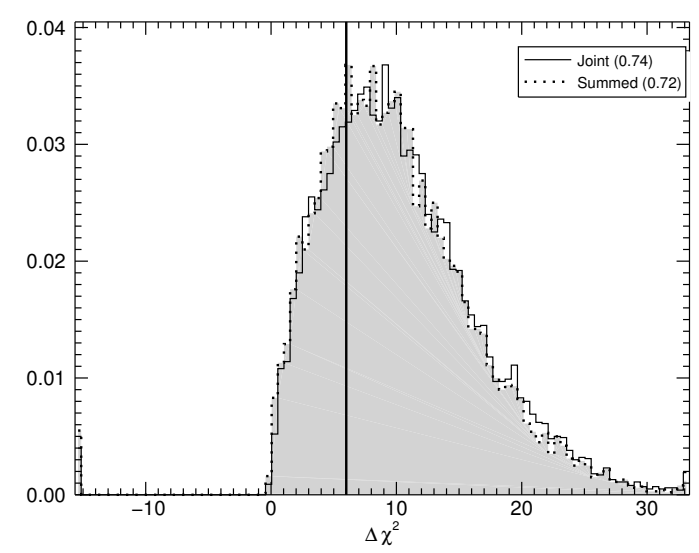

(c)

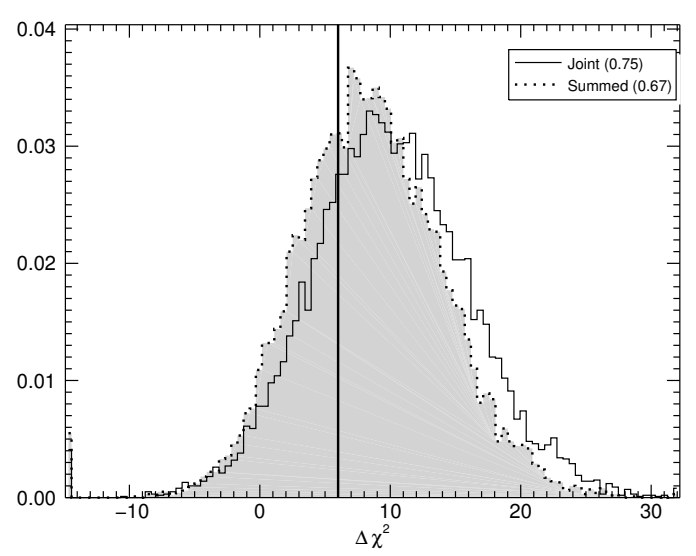

(b)

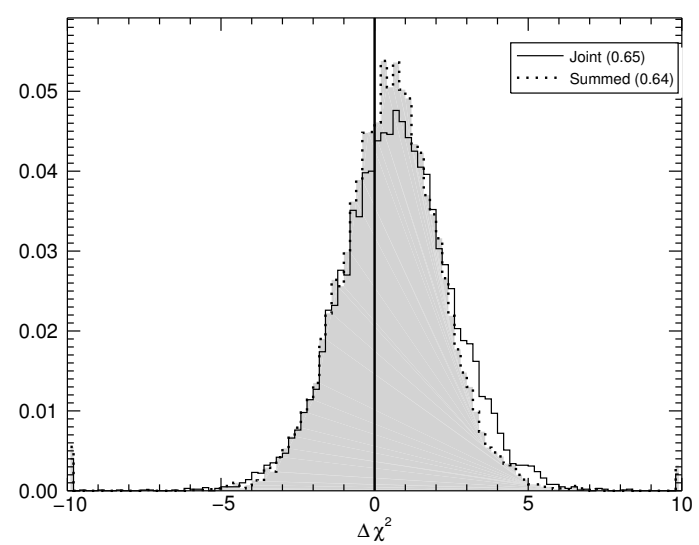

(d)

Fig. 37.- Comparisons of the $\Delta \chi^{2}$ distributions between the joint and summed simulations. 10,000 simulated BAND spectra were created and the other four models were fit to the simulated data. The vertical lines in each plot represent the cutoffs specified by our model selection criteria. To determine if the model selections change between the summed and joint data, we measure how much of each distribution exists above the cutoff. This indicates a probability that our cutoff will prefer a BAND model rather than the other model. In all cases the distributions are different, but approximately the same amount of each distribution exists above the cutoff (percentages in parentheses). 
Table 6. K-S Test between Fluence and Peak Flux Parameters

\begin{tabular}{ccc}
\hline Parameter & K-S Stat & Probability \\
\hline PL Index & 0.14 & $2.3 \times 10^{-19}$ \\
SBPL Alpha & 0.20 & $3.1 \times 10^{-31}$ \\
BAND Alpha & 0.23 & $1.0 \times 10^{-27}$ \\
COMP Index & 0.24 & $5.9 \times 10^{-44}$ \\
\hline SBPL Beta & 0.03 & 0.68 \\
BAND Beta & 0.05 & 0.15 \\
\hline SBPL $E_{\text {peak }}$ & 0.09 & $5.5 \times 10^{-5}$ \\
BAND $E_{\text {peak }}$ & 0.09 & $9.3 \times 10^{-6}$ \\
COMP $E_{\text {peak }}$ & 0.09 & $1.1 \times 10^{-6}$ \\
GLOG $E_{\text {peak }}$ & 0.16 & $3.1 \times 10^{-7}$ \\
\hline \hline
\end{tabular}

Table 7. BEST GRB models

\begin{tabular}{ccccc}
\hline PL & SBPL & BAND & COMP & GLOGE \\
\hline \multicolumn{5}{c}{ Fluence Spectra } \\
\hline $506(23 \%)$ & $124(6 \%)$ & $77(4 \%)$ & $903(42 \%)$ & $535(25 \%)$ \\
\hline \multicolumn{5}{c}{ Peak Flux Spectra } \\
\hline $454(21 \%)$ & $150(7 \%)$ & $65(3 \%)$ & $847(40 \%)$ & $629(29 \%)$ \\
\hline
\end{tabular}


Table 8. Sample mean and standard deviation of the parameter distributions

\begin{tabular}{|c|c|c|c|c|c|c|c|c|}
\hline \multirow{2}{*}{ Model } & \multirow{2}{*}{$\begin{array}{c}\text { Low-Energy } \\
\text { Index }\end{array}$} & High-Energy & \multirow{2}{*}{$\mathbf{E}_{\text {peak }}(\mathbf{k e V})$} & \multirow{2}{*}{$\mathbf{E}_{\text {break }}(\mathbf{k e V})$} & \multirow[t]{2}{*}{$\mathbf{E}_{\text {cent }}(\mathrm{keV})$} & \multirow{2}{*}{$\begin{array}{c}\text { FWHM } \\
\left(\log _{10} \mathrm{keV}\right)\end{array}$} & \multirow{2}{*}{ Photon Flux } & \multirow{2}{*}{$\begin{array}{l}\text { Energy Flux } \\
\left.-7 \mathrm{erg} \mathrm{s}^{-1} \mathrm{~cm}^{-2}\right)\end{array}$} \\
\hline & & Index & & & & & & \\
\hline \multicolumn{9}{|c|}{ Fluence Spectra } \\
\hline COMP & $-1.12_{-0.43}^{+0.55}$ & - & $195.11_{-98.74}^{+256.43}$ & - & - & - & $0.68_{-0.33}^{+0.80}$ & $1.22_{-0.62}^{+1.96}$ \\
\hline SBPL & $-1.36_{-0.52}^{+0.47}$ & $-2.62_{-0.71}^{+0.56}$ & $190.77_{-92.77}^{+186.24}$ & $173.18_{-88.64}^{+150.14}$ & - & - & $0.71_{-0.35}^{+0.93}$ & $1.50_{-0.76}^{+2.47}$ \\
\hline GLOGE & - & - & $131.53_{-59.93}^{+161.72}$ & - & $12.34_{-7.25}^{+24.82}$ & $1.22_{-0.27}^{+0.30}$ & $0.60_{-0.29}^{+0.67}$ & $1.14_{-0.56}^{+1.68}$ \\
\hline BEST & $-1.29_{-0.63}^{+0.60}$ & $-2.53_{-0.71}^{+0.57}$ & $195.94_{-104.32}^{+267.66}$ & $195.78_{-120.56}^{+191.39}$ & $13.99_{-7.15}^{+22.07}$ & $1.10_{-0.24}^{+0.24}$ & $0.75_{-0.37}^{+1.09}$ & $1.40_{-0.71}^{+2.49}$ \\
\hline \multicolumn{9}{|c|}{ Peak Flux Spectra } \\
\hline $\mathrm{PL}$ & $-1.72_{-0.39}^{+0.32}$ & - & - & - & - & - & $0.80_{-0.40}^{+0.78}$ & $1.91_{-0.85}^{+1.48}$ \\
\hline GLOGE & - & - & $156.18_{-73.65}^{+189.15}$ & - & $20.68_{-12.72}^{+29.45}$ & $1.18_{-0.28}^{+0.33}$ & $1.19_{-0.73}^{+1.96}$ & $2.62_{-1.53}^{+4.80}$ \\
\hline BEST & $-1.10_{-0.70}^{+0.62}$ & $-2.69_{-0.73}^{+0.46}$ & $228.25_{-119.73}^{+271.74}$ & $273.38_{-128.46}^{+196.39}$ & $21.92_{-12.53}^{+27.75}$ & $1.09_{-0.24}^{+0.28}$ & $1.61_{-1.02}^{+4.32}$ & $3.44_{-2.09}^{+11.5}$ \\
\hline
\end{tabular}

Table 9. Comparison of the sample mean and standard deviation from different catalogs

\begin{tabular}{|c|c|c|c|c|c|c|}
\hline Dataset & $\begin{array}{c}\text { Low-Energy } \\
\text { Index }\end{array}$ & $\begin{array}{l}\text { High-Energy } \\
\text { Index }\end{array}$ & $\underset{(\mathrm{keV})}{\mathrm{E}_{\text {peak }}}$ & $\underset{(\mathrm{keV})}{\mathbf{E}_{\text {break }}}$ & $\begin{array}{c}\text { Photon Flux } \\
\left(\mathrm{ph} \mathrm{s}^{-1} \mathbf{c m}^{-2}\right)\end{array}$ & $\begin{array}{c}\text { Energy Flux } \\
\left(10^{-7} \mathrm{erg} \mathrm{s}^{-1} \mathrm{~cm}^{-2}\right)\end{array}$ \\
\hline \multicolumn{7}{|c|}{ Fluence } \\
\hline This Catalog BEST & $-1.29_{-0.63}^{+0.60}$ & $-2.53_{-0.71}^{+0.57}$ & $196_{-104}^{+268}$ & $196_{-121}^{+191}$ & $0.75_{-0.37}^{+1.09}$ & $1.40_{-0.71}^{+2.49}$ \\
\hline Goldstein et al. (2012) & $-1.05_{-0.45}^{+0.44}$ & $-2.25_{-0.73}^{+0.34}$ & $205_{-121}^{+359}$ & $123_{-80.4}^{+240}$ & $2.92_{-1.31}^{+3.96}$ & $4.03_{-2.13}^{+9.38}$ \\
\hline Kaneko et al. (2006) & $-1.07_{-0.36}^{+0.42}$ & $-2.43_{-0.59}^{+0.38}$ & $260_{-116}^{+233}$ & $203_{-80.0}^{+129}$ & $3.32_{-2.04}^{+6.01}$ & $8.56_{-5.47}^{+16.0}$ \\
\hline \multicolumn{7}{|c|}{ Peak Flux Spectra } \\
\hline This Catalog BEST & $-1.10_{-0.70}^{+0.62}$ & $-2.69_{-0.73}^{+0.46}$ & $228_{-120}^{+271}$ & $273_{-128}^{+196}$ & $1.61_{-1.02}^{+4.32}$ & $3.44_{-2.09}^{+11.5}$ \\
\hline Goldstein et al. (2012) & $-1.12_{-0.50}^{+0.61}$ & $-2.27_{-0.50}^{+0.44}$ & $223_{-126}^{+352}$ & $172_{-100}^{+254}$ & $5.39_{-2.87}^{+10.18}$ & $8.35_{-4.98}^{+22.61}$ \\
\hline
\end{tabular}


Table 10. Mean and Standard Deviation of Simulated Parameters

\begin{tabular}{cccc}
\hline Parameter & Catalog & Joint Sim. & Summed Sim. \\
\hline \multicolumn{4}{c}{ GRB 910425 - PL } \\
\hline Index & $-1.678 \pm 0.043$ & $-1.677_{-0.044}^{+0.040}$ & $-1.678_{-0.045}^{+0.042}$ \\
\hline \multicolumn{4}{c}{ GRB 910521 - GLOGE } \\
\hline$E_{\text {cen }}$ & $12.094 \pm 4.758$ & $12.428_{-3.402}^{+4.149}$ & $12.690_{-3.482}^{+4.310}$ \\
FWHM & $0.904 \pm 0.096$ & $0.898_{-0.076}^{+0.075}$ & $0.893_{-0.077}^{+0.074}$ \\
\hline \multicolumn{4}{c}{ GRB 910426 - COMP } \\
\hline$E_{\text {peak }}$ & $93.182 \pm 2.956$ & $93.163_{-3.030}^{+2.842}$ & $92.129_{-3.089}^{+2.878}$ \\
$\alpha$ & $-1.095 \pm 0.109$ & $-1.093_{-0.106}^{+0.110}$ & $-1.096_{-0.107}^{+0.113}$ \\
\hline \multicolumn{5}{c}{ GRB 910421 - BAND } \\
\hline$E_{\text {peak }}$ & $127.788 \pm 3.334$ & $127.692_{-5.700}^{+3.253}$ & $127.690_{-6.562}^{+3.354}$ \\
$\alpha$ & $-0.996 \pm 0.060$ & $-0.995_{-0.054}^{+0.076}$ & $-0.995_{-0.057}^{+0.090}$ \\
$\beta$ & $-2.939 \pm 0.171$ & $-2.938_{-0.388}^{+0.158}$ & $-2.940_{-0.377}^{+0.168}$ \\
\hline \multicolumn{5}{c}{ GRB 910522 - SBPL } \\
\hline$E_{\text {break }}$ & $224.585 \pm 14.324$ & $224.826_{-13.630}^{+14.734}$ & $224.685_{-13.651}^{+14.585}$ \\
$\alpha$ & $-1.343 \pm 0.019$ & $-1.343_{-0.018}^{+0.020}$ & $-1.343_{-0.018}^{+0.020}$ \\
$\beta$ & $-2.461 \pm 0.058$ & $-2.462_{-0.062}^{+0.052}$ & $-2.460_{-0.063}^{+0.052}$ \\
\hline \hline
\end{tabular}

